CERN-PH-TH/2010-130

SU-ITP-10/08

UCB-PTH-10/12

\title{
Charge Orbits of Extremal Black Holes in Five Dimensional Supergravity
}

\author{
Bianca L. Cerchiai ${ }^{\sharp}$, Sergio Ferrara ${ }^{\natural, b}$, \\ Alessio Marrani ${ }^{\triangleright}$ and Bruno Zumino ${ }^{\star} \diamond$ \\ $\sharp$ Università degli Studi di Milano, \\ Dipartimento di Matematica "Federigo Enriques" \\ Via Cesare Saldini 50, I-20133 Milano, Italy \\ Bianca.Cerchiai@unimi.it \\ \& Theory division, CERN, Geneva, Switzerland \\ CH 1211, Geneva 23, Switzerland \\ sergio.ferrara@cern.ch \\ b INFN - LNF, \\ Via Enrico Fermi 40, I-00044 Frascati, Italy \\ $\odot$ Stanford Institute for Theoretical Physics \\ Stanford University, Stanford, CA 94305-4060, USA \\ marrani@lnf. infn. it \\ a Lawrence Berkeley National Laboratory, \\ Theory Group, Bldg 50A5104 \\ 1 Cyclotron Rd, Berkeley, CA 94720-8162, USA \\ zumino@thsrv. lbl.gov \\ $\diamond$ Department of Physics, University of California, \\ Berkeley, CA 94720-8162, USA
}

\begin{abstract}
We derive the $U$-duality charge orbits, as well as the related moduli spaces, of "large" and "small" extremal black holes in non-maximal ungauged Maxwell-Einstein supergravities with symmetric scalar manifolds in $d=5$ space-time dimensions.

The stabilizer groups of the various classes of orbits are obtained by determining and solving suitable $U$-invariant sets of constraints, both in "bare" and "dressed" charges bases, with various methods.

After a general treatment of attractors in real special geometry (also considering non-symmetric cases), the $\mathcal{N}=2$ "magic" theories, as well as the $\mathcal{N}=2$ Jordan symmetric sequence, are analyzed in detail. Finally, the half-maximal $(\mathcal{N}=4)$ matter-coupled supergravity is also studied in this context.
\end{abstract}




\section{Contents}

1 Introduction $\quad 2$

2 Résumé of Real Special Geometry (RSG) 3

3 Attractors in RSG $\quad 6$

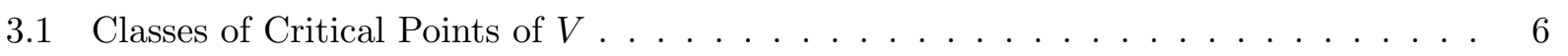

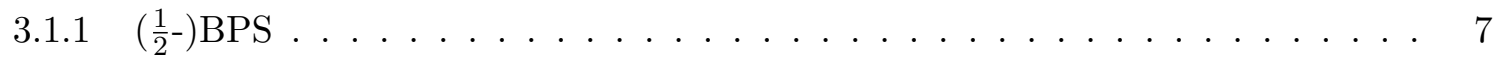

3.1 .2 Non-BPS . . . . . . . . . . . . . . . . . . 7

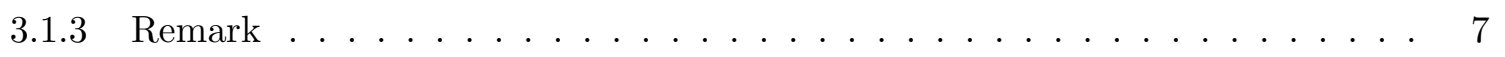

3.1.4 "New Attractor" Approach . . . . . . . . . . . . . . 8

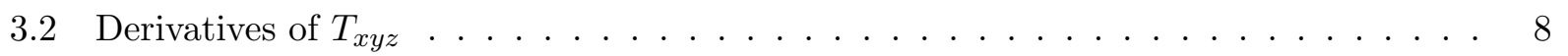

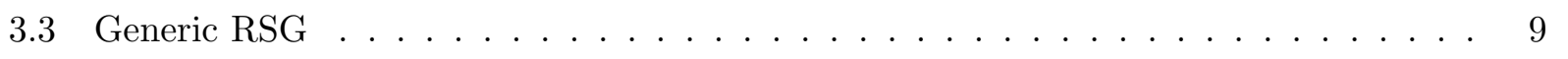

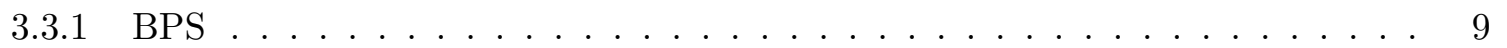

3.3.2 Non-BPS and the "Dressed" Charges' Sum Rule . . . . . . . . . . . . 9

3.3 .3 The Functional $\widehat{\mathcal{I}}_{3} \ldots \ldots \ldots \ldots \ldots \ldots$. . . . . . . . . . . . . . . . . . . . . . . .

3.4 Symmetric RSG and "Large" Charge Orbits . . . . . . . . . . . . . . . . 11

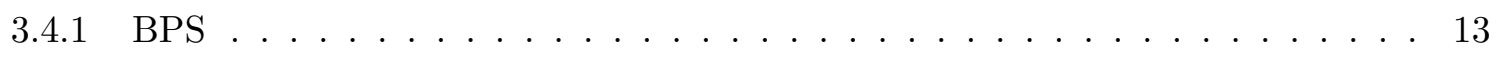

3.4 .2 Non-BPS . . . . . . . . . . . . . . . . . . . . 14

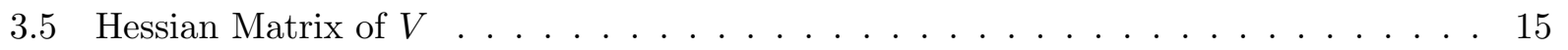

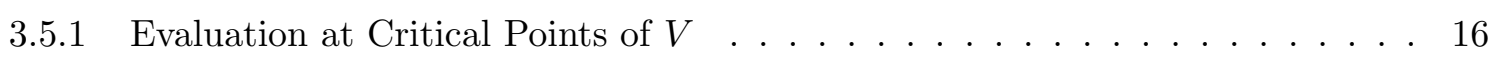

4 "Small" Charge Orbits and Moduli Spaces in Symmetric "Magic" RSG 17

$4.1 G_{5}$-invariant Defining Constraints . . . . . . . . . . . . . . . 22

4.1 .1 "Small" Lightlike Orbits . . . . . . . . . . . . . . . . . . . . . . . . . . . .

4.1 .2 "Small" Critical Orbit ........................ 27

$5 J_{3}^{\mathbb{H}}: \mathcal{N}=2$ versus $\mathcal{N}=6$

$6 \mathcal{N}=2, d=5$ Jordan Symmetric Sequence 28

6.1 "Large" Orbits . . . . . . . . . . . . . . . . . . . . . . . 30

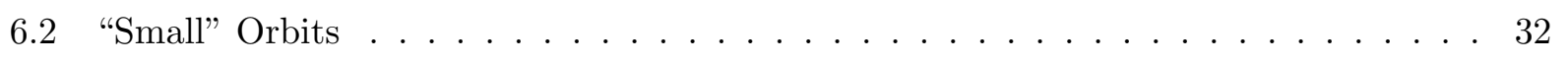

$7 \mathcal{N}=4, d=5$ Supergravity 37

7.1 "Large" Orbits . . . . . . . . . . . . . . . . . . . . . . . 37

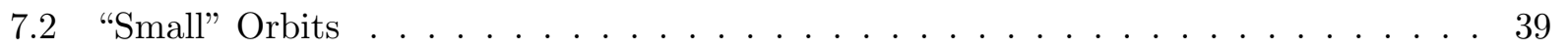

A Resolution of $G_{5}$-invariant Constraints 44

A.1 "Bare" Charges Basis . . . . . . . . . . . . . . . . . . . . 44

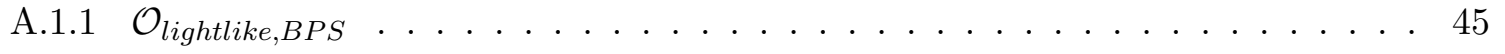

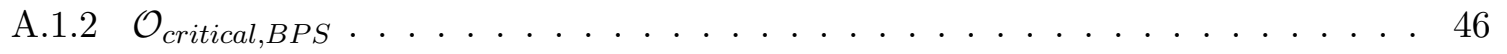

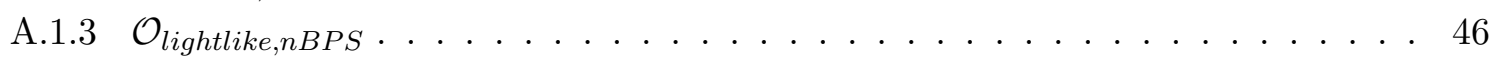

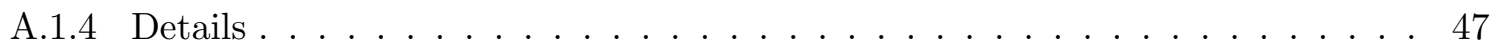


A.2 "Dressed" Charges Basis . . . . . . . . . . . . . . . . . . 50

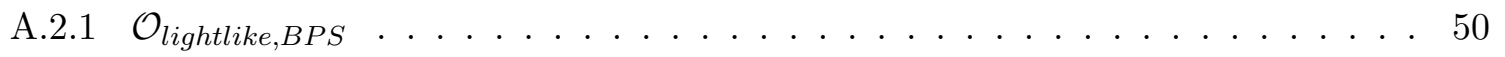

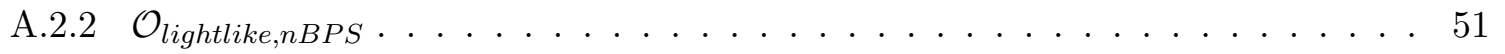

B Equivalent Derivations

B.1 İönü-Wigner Contractions . . . . . . . . . . . . . . . . . . . 5 53

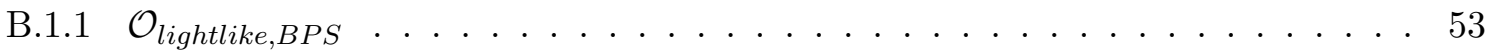

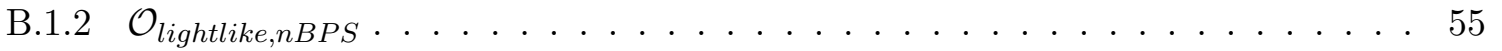

B.1.3 Details . . . . . . . . . . . . . . . . . . . 555

B.2 $S O(1,1)$-Three Grading and $\mathcal{O}_{\text {critical }, B P S} \ldots \ldots \ldots \ldots \ldots$

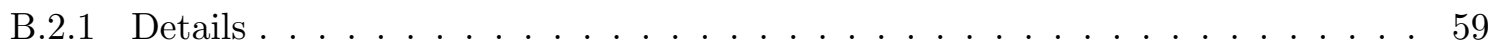

\section{Introduction}

Five-dimensional supergravity theories with non-maximal supersymmetry $(2 \leqslant \mathcal{N}<8)$, emerging from Calabi-Yau compactifications of $M$-theory, admit extremal black $p$-brane solutions in their spectrum [1. In particular, ungauged theories admit extremal black holes $(p=0)$ and black strings $(p=1)$ which are asymptotically flat, and reciprocally related through $U$-duality 1 . These objects have been intensely studied along the years, due to the wide range of classical and quantum aspects they exhibit.

For asymptotically flat, spherically symmetric and stationary solutions, the Attractor Mechanism [3, 4, 5, 6] proved to be a crucial phenomenon, determining, in a universal fashion, the stabilization of scalar fields in the near-horizon geometry in terms of the fluxes of the two-form field strengths of the Abelian vector fields coupled to the system. Moreover, the Attractor Mechanism turned out to be important also to unravel dynamical properties such as split attractor flows [7] and wall crossing [8], and to gain insights in the microstate counting analysis (see e.g. [9], and Refs. therein), also in relation to string topological partition functions 10] (see also 11] for a recent account and list of Refs.). In $d=5$ space-time dimensions, progress has been achieved also with the discovery of new attractor solutions (see e.g. [12]), as well as with the formulation of a first-order formalism governing the evolution dynamics of non-supersymmetric scalar flows [13].

For supergravity theories with scalar manifolds which are symmetric cosets, the extremal solutions of the ungauged theory can be classified through the orbits of the relevant representation space of the $U$-duality group, in which the corresponding supporting charges sit. The relation between $U$-invariant BPS conditions and charge orbits in $d=5$ supergravities has been the subject of various studies along the years [14, 15, 16, 17, 18, 19, 20.

The present paper extends to $d=5$ space-time dimensions the 4-dimensional investigation of 21.

We derive the $U$-duality charge orbits, as well as the related moduli spaces, of "large" and "small" extremal black holes and black strings in ungauged Maxwell-Einstein supergravities with symmetric scalar manifolds. The stabilizer groups of the various classes of orbits are obtained by determining and solving suitable $U$-invariant sets of constraints, both in "bare" and "dressed" charges bases, as well by exploiting İnönü-Wigner contractions and $S O(1,1)$-gradings.

It is here worth pointing out that in this paper we will not deal with maximal $\mathcal{N}=8, d=5$

\footnotetext{
${ }^{1}$ Here $U$-duality is referred to as the "continuous" version, valid for large values of the charges, of the $U$-duality groups introduced by Hull and Townsend [2].
} 
supergravity, because a complete analysis of extremal black hole attractors and their "large" and "small" charge orbits is already present in literature, see e.g. [17, 14, 15, 16, 18, 22, 23, 20, 24, 25, 26. We will just mention such a theory shortly below Eq. (4.3).

The plan of the paper is as follows.

We start and give a résumé of real special geometry in Sect. 2, setting up notation and presenting all formulæ needed for the subsequent treatment of charge orbits and attractors.

In Sect. 3 extremal black hole (black string) attractors are studied in full generality within real special geometry. Starting from the treatment of [19], various refinements and generalizations are performed, in particular addressing the issue of generic, non-symmetric vector multiplets' scalar manifolds. In Subsect. 3.1 we analyze the various classes of critical points of the effective potential $V$, also within the so-called "new attractor" approach (see Subsubsect. 3.1.4). Then, in Subsect. 3.2 we compute the higher order covariant derivatives of the previously introduced rank-3 invariant tensor $T_{x y z}$, which will play a key role in the subsequent developments and results, exposed in Subsects. 3.3 and 3.4 , respectively dealing with generic and homogeneous symmetric real special manifolds. A general analysis of the Hessian matrix of $V$, crucial in order to establish the stability of considered attractor points, is then performed in Subsect. 3.5.

In Sect. 4 all "small" charge orbits of symmetric "magic" real special geometries are explicitly determined and classified, by exploiting the properties of the functional $\widehat{\mathcal{I}}_{3}$ introduced in Subsubsect. 3.3.3. Note that "small" charge orbits support non-attractor solutions, which have vanishing Bekenstein-Hawking [27] entropy in the Einsteinian approximation. Nevertheless, they can be treated by exploiting their symmetry properties under $U$-duality.

Sect. 5 analyzes the "duality" relating the $\mathcal{N}=2$ "magic" theory coupled to 14 Abelian vector multiplets and the $\mathcal{N}=6$ "pure" supergravity, both based on the rank-3 Euclidean Jordan algebra $J_{3}^{\mathbb{H}}$ and thus sharing the very same bosonic sector.

Then, Sect. 6 is devoted to the analysis of the "large" (Subsect. 6.1) and "small" (Subsect. 6.2) charge orbits of $\mathcal{N}=2$ Jordan symmetric sequence. Similarly, Sect. 7 provides a detailed treatment of the "large" (Subsect. 7.1) and "small" (Subsect. 7.2) charge orbits of the half-maximal $(\mathcal{N}=4)$ matter coupled supergravity. The analysis of both Sects. 6 and 7 is made in the "bare" charges basis, and various subtleties, related to the reducible nature of the $d=5 U$-duality group and disconnectedness of orbits in these two theories, are elucidated.

Some Appendices conclude the paper, containing various details concerning the determination of the "small" orbits in symmetric "magic" real special geometries.

The resolution of $U$-invariant defining (differential) constraints, both in "bare" and "dressed" charges bases, is performed in App. A.

Then, in App. B we give an equivalent derivation of all "small" charge orbits of symmetric "magic" real special geometries, relying on group theoretical procedures, namely İnönü-Wigner contractions (Sub-App. B.1) and $S O(1,1)$-three-grading (Sub-App. B.2).

Finally, we point out that all results on charge orbits can actually be obtained in various other ways, including the analysis of cubic norm forms of the relevant Jordan systems in $d=5$; this will be investigated elsewhere.

\section{Résumé of Real Special Geometry (RSG)}

Real special geometry (RSG) ([28, 29, 30, 31, 32, 33], and Refs. therein) is the geometry underlying the scalar manifold $M_{5}$ (with Euclidean signature) of Abelian vector multiplets coupled to the 
minimal supergravity in $d=5$ space-time dimensions, namely to $\mathcal{N}=2, d=5$ theory.

In the present Section, we recall some basic facts about RSG, setting up notation and presenting all formulæ needed for the subsequent treatment of charge orbits and attractors. Apart from a slight changes in notation, we will adopt the conventions of [19], which are slightly different from the ones used in 34] (see the observations in [34] itself).

We start by specifying the kind and range of indices being used. $i=0,1, \ldots n_{V}$ is the index in the "ambient space" (in which $M_{5}$ is defined through a cubic constraint; see Eq. 2.5 below). "0" is the index pertaining to the ("bare") $d=5$ graviphoton, and $n_{V}$ stands for the number of Abelian vector multiplets coupled to the supergravity multiplet. On the other hand, $x=1, \ldots, n_{V}$, and $a=1, \ldots, n_{V}$ respectively denote "curved" and (local) "flat" coordinates in $M_{5}$.

The metric $a_{i j}$ in the "ambient space" (named $g_{i j}$ in [34]) can be defined as follows:

$$
a_{i j}=-\frac{1}{3} \frac{\partial^{2} \log \mathcal{V}(\lambda)}{\partial \lambda^{i} \partial \lambda^{j}}
$$

where

$$
\mathcal{V}(\lambda) \equiv d_{i j k} \lambda^{i} \lambda^{j} \lambda^{k}>0
$$

is the volume of $M_{5}$ itself, and $d_{i j k}=d_{(i j k)}$ is a rank-3 completely symmetric invariant tensor (see further below). In turn, the $\lambda^{i}$ 's are some real functions (with suitable features of smoothness and regularity) of the set of scalars $\phi^{x}$ of the theory, coordinatizing $M_{5}$ :

$$
\lambda^{i}=\lambda^{i}\left(\phi^{x}\right) .
$$

They do satisfy the inequality 2.2). As elucidated e.g. in [34], the $\lambda^{i}$ 's are nothing but the (opposite of the) imaginary ("dilatonic") part of the complex scalar fields of the special Kähler geometry (SKG) based on a cubic holomorphic prepotential (usually named $d$-SKG; see e.g. [32, 36]), endowing the Abelian vector multiplets' scalar manifold of $\mathcal{N}=2$ Maxwell-Einstein supergravity in 4 space-time dimensions. In this respect, the "ambient space" in 5 dimensions is nothing but the "dilatonic sector" of the $d$-SKG in 4 dimensions.

It is now convenient to introduce rescaled variables as follows:

$$
\widehat{\lambda}^{i} \equiv \lambda^{i} \mathcal{V}^{-1 / 3} \Leftrightarrow d_{i j k} \widehat{\lambda}^{i} \widehat{\lambda}^{j} \widehat{\lambda}^{k}=\mathcal{V}(\widehat{\lambda})=1
$$

Thus, the metric of $M_{5}$ is the pull-back of $a_{i j}$ on the hypersurface

$$
\mathcal{V}(\lambda) \equiv 1
$$

in the "ambient space", namely:

$$
\left.g_{x y} \equiv \widehat{\lambda}_{x}^{i} \widehat{\lambda}_{y}^{j} a_{i j}\right|_{\mathcal{V}(\lambda) \equiv 1}=-\left.\frac{1}{3} \widehat{\lambda}_{x}^{i} \widehat{\lambda}^{j}{ }_{y} \frac{\partial^{2} \log \mathcal{V}(\lambda)}{\partial \lambda^{i} \partial \lambda^{j}}\right|_{\mathcal{V}(\lambda) \equiv 1}=g_{x y}(\widehat{\lambda}(\phi))=\stackrel{(\sim)}{g}_{x y}(\phi),
$$

where (the semicolon denotes Riemann-covariant differentiation throughout)

$$
\widehat{\lambda}_{x}^{i} \equiv-\sqrt{\frac{3}{2}} \frac{\partial \widehat{\lambda}^{i}}{\partial \phi^{x}} \equiv-\sqrt{\frac{3}{2}} \widehat{\lambda}_{, x}^{i}=-\sqrt{\frac{3}{2}} \widehat{\lambda}_{; x}^{i} .
$$

Notice that the constraint (2.4) implies

$$
\frac{\partial \mathcal{V}(\widehat{\lambda})}{\partial \phi^{x}}=3 d_{i j k} \widehat{\lambda}_{, x}^{i} \widehat{\lambda}^{j} \widehat{\lambda}^{k}-\sqrt{6} d_{i j k} \widehat{\lambda}^{i}{ }_{x} \widehat{\lambda}^{j} \widehat{\lambda}^{k}=0 .
$$


Let us now introduce $T_{x y z}$, a rank-3 completely symmetric invariant tensor, related to $d_{i j k}$ through the definition

$$
T_{x y z} \equiv \widehat{\lambda}_{x}^{i} \widehat{\lambda}_{y}^{j} \widehat{\lambda}^{k}{ }_{z} d_{i j k}=-\left(\frac{3}{2}\right)^{3 / 2} \widehat{\lambda}_{, x}^{i} \widehat{\lambda}_{, y}^{j} \widehat{\lambda}_{, z}^{k} d_{i j k}=T_{(x y z)}
$$

whose inversion reads

$$
d_{i j k}=\frac{5}{2} \widehat{\lambda}_{i} \widehat{\lambda}_{j} \widehat{\lambda}_{k}-\frac{3}{2} \stackrel{\circ}{(i j}_{(i j} \widehat{\lambda}_{k)}+T_{x y z} \widehat{\lambda}_{i}{ }^{x} \widehat{\lambda}_{j}{ }^{y} \widehat{\lambda}_{k}^{z}
$$

where

$$
\left.\stackrel{\circ}{a}_{i j}(\widehat{\lambda}) \equiv a_{i j}\right|_{\mathcal{V}(\lambda) \equiv 1} .
$$

In other words, $T_{x y z}$ is the $\phi$-dependent "dressing" (through $\widehat{\lambda}_{, x}^{i}(\phi)$ 's) of the constant ( $\phi$-independent) tensor $d_{i j k}$. It is here worth anticipating that Eqs. 2.9) and (2.10) play the key role to relate the formalism based on "bare" charges with the formalism based on the "dressed" charges (see further below).

$T_{x y z}$ enters the so-called "RSG constraints", relating in $M_{5}$ the Riemann tensor $R_{x y z u}$ to the metric tensor $g_{x y}$, as follows:

$$
R_{x y z u}=\frac{4}{3}\left(g_{x[u} g_{z] y}+T_{x[u}{ }^{w} T_{z] y w}\right)=\frac{4}{3}\left(g_{x[u} g_{z] y}+T_{x w^{\prime}[u} T_{z] y w} g^{w w^{\prime}}\right) .
$$

It is worth noticing a direct consequence of such "RSG constraints": the sectional curvature (see e.g. [37] and [38]) of matter charges in RSG globally vanishes:

$$
\mathcal{R}(Z) \equiv R_{x y z w} g^{x x^{\prime}} g^{y y^{\prime}} g^{z z^{\prime}} g^{w w^{\prime}} Z_{x^{\prime}} Z_{y^{\prime}} Z_{z^{\prime}} Z_{w^{\prime}}=0 .
$$

This is trivially due to the symmetry properties of the Riemann tensor $R_{x y z w}$ (which are the ones for a generic Riemann geometry), and it is a feature discriminating RSG from SKG (in which $\mathcal{R}(Z)$ generally does not vanish; see e.g. [39, 40]).

As a consequence of the constraints (2.12) (within the metric postulate), the definition of $M_{5}$ to be an homogeneous symmetric manifold

$$
R_{x y z u ; t}=0
$$

yields

$$
\left(T_{x w^{\prime}[u ; t} T_{z] y w}+T_{x w^{\prime}[u} T_{z] y w ; t}\right) g^{w w^{\prime}}=0 \Leftrightarrow T_{x w^{\prime}[u} T_{z] y w ; t} g^{w w^{\prime}}=T_{x w[u} T_{z] y ; t}^{w}=0,
$$

solved by

$$
T_{x y z ; u}=0 .
$$

Through Eqs. 2.9) and 2.10), and exploiting the constraints imposed by local $\mathcal{N}=2$ supersymmetry, it can be shown that Eq. (2.16) implies the following relation between the tensors $d_{i j k}$ :

$$
d^{i j k} d_{j(m n} d_{p q) k}=\delta_{(m}^{i} d_{n p q)} \Leftrightarrow d_{j(m n} d_{p q) k} d_{r s t} \stackrel{\circ}{a j} \stackrel{a}{a} \stackrel{\circ}{a} \stackrel{\circ}{a}=\delta_{(m}^{i} d_{n p q)},
$$

where the index-raising through the contravariant metric $\stackrel{\circ}{a}^{i j}$ has been explicited. 


\section{Attractors in RSG}

The present Section is dedicated to the study of attractors in RSG. This has been firstly treated in [19] (and then reconsidered in [20], in connection to $d=6$ ).

Starting from the treatment of [19], we will generalize and elaborate further various results obtained therein.

It is worth recalling that no asymptotically-flat dyonic solutions of Einstein Eqs. exist in $d=5$. Thus, the $d=5$ asymptotically flat black holes (BHs) can only carry electric charges $q_{i}$. Their magnetic duals are the $d=5$ asymptotically flat black strings, which can only carry magnetic charges $p^{i}$.

We will perform all our treatment within the electric charge configuration. Due to the mentioned $\mathrm{BH} /$ black string duality, this does not imply any loss of generality. Furthermore, we will study attractors within the Ansätze of asymptotical (Minkowski) flatness, staticity, spherical symmetry and extremality of the $\mathrm{BH}$ space-time metric (if no scalars are coupled, this is nothing but the so-called Tangherlini extremal $d=5 \mathrm{BH}$ ). The near-horizon geometry of extremal electric $\mathrm{BHs}$ and extremal magnetic black strings respectively is $A d S_{2} \times S^{3}$ and $A d S_{3} \times S^{2}$.

\subsection{Classes of Critical Points of $V$}

From the general theory of Attractor Mechanism [3, 4, 5, 6], the stabilization of scalar fields in proximity of the (unique) event horizon of a static, spherically symmetric and asymptotically flat extremal $\mathrm{BH}$ in $\mathcal{N}=2, d=5$ Maxwell-Einstein supergravity is described by the critical points of the positive-definite effective potential function

$$
V \equiv \stackrel{\circ i j}{a} q_{i} q_{j}=\left(\widehat{\lambda}^{i} q_{i}\right)^{2}+\frac{3}{2} g^{x y} \widehat{\lambda}_{, x}^{i} q_{i} \widehat{\lambda}_{, y}^{j} q_{j}=Z^{2}+\frac{3}{2} g^{x y} Z_{x} Z_{y}
$$

where the $\mathcal{N}=2, d=5$ central charge function $Z$ and its Riemann-covariant derivatives ( $m a t t e r$ charges") have been defined as follows:

$$
\begin{aligned}
Z & \equiv \widehat{\lambda}^{i} q_{i} ; \\
Z_{x} & \equiv \widehat{\lambda}_{, x}^{i} q_{i}=Z_{, x}=Z_{; x} .
\end{aligned}
$$

The definitions (3.2) and (3.3) can be inverted, obtaining the fundamental identities of RSG (in electric formulation) [19]:

$$
q_{i}=\widehat{\lambda}_{i} Z-\frac{3}{2} g^{x y} \widehat{\lambda}_{i, x} Z_{y} .
$$

The identities (3.4) relate the basis of "bare" ( $\phi$-independent) electric charges $q_{i}$ to the basis of "dressed" (central and matter) charges $\left\{Z, Z_{x}\right\}$, which do depend on the scalars $\phi^{x}$, as yielded by definitions 3.2 and 3.3 .

By recalling definitions 3.2 and $(3.3)$, one obtains that

$$
Z_{x y} \equiv Z_{x ; y}=Z_{, x ; y}=Z_{; x ; y}=\widehat{\lambda}_{, x ; y}^{i} q_{i}=\frac{2}{3} g_{x y} Z-\sqrt{\frac{2}{3}} T_{x y z} g^{z w} Z_{w} .
$$

Therefore, by using Eq. (3.5) the criticality conditions (alias Attractor Eqs.) for the effective potential $V$ can be easily computed to be [19]:

$$
V_{x} \equiv V_{, x}=V_{; x}=2\left(2 Z Z_{x}-\sqrt{\frac{3}{2}} T_{x y z} g^{y s} g^{z t} Z_{s} Z_{t}\right)=0 .
$$


A priori, the classes of critical points of $V$ which are non-degenerate (i.e. with $\left.V\right|_{V_{x}=0} \neq 0$ ) are three:

\subsection{1 ( $\left.\frac{1}{2}-\right)$ BPS}

This class is defined by the sufficient (but not necessary) criticality constraint

$$
Z_{x}=0, \forall x
$$

implying

$$
V=Z^{2}
$$

\subsubsection{Non-BPS}

This class is defined by the constraints

$$
\left\{\begin{array}{l}
Z \neq 0 \\
Z_{x} \neq 0 \text { for at least some } x \text { 's, }
\end{array}\right.
$$

which are critical provided the following algebraic constraint among $Z$ and $Z_{x}$ 's hold:

$$
Z_{x}=\frac{1}{2 Z} \sqrt{\frac{3}{2}} T_{x y z} g^{y s} g^{z t} Z_{s} Z_{t} .
$$

At least in symmetric RSG, this implies [19]

$$
V=9 Z^{2} .
$$

\subsubsection{Remark}

It is here worth recalling the Bekenstein-Hawking entropy-area formula [27], implemented for critical points of $V$ :

$$
\frac{S_{B H, d=5}}{\pi}=\frac{A_{H}}{4 \pi} \equiv R_{H}^{2}=\left(\left.V\right|_{\partial V=0}\right)^{3 / 4} .
$$

The Attractor Mechanism [3, 4, 5, 6] is known to hold only for the so-called "large" BHs, which, through Eq. (3.12), have a non-vanishing Bekenstein-Hawking entropy.

Therefore, attractors in strict sense are given by non-degenerate critical points of $V$. On the other hand, degenerate critical points of $V$, namely critical points such that $\left.V\right|_{\partial V=0}=0$ are trivial. Indeed, by virtue of the positive definiteness of $V$ (inherited from the strictly positive definiteness of $\stackrel{o i j}{a}^{i j}$ throughout all its domain of definition), it holds that

$$
V=0 \Leftrightarrow q_{i}=0 \forall i,
$$

which is the trivial limit of the theory with all (electric) charges switched off.

The same reasoning can be repeated in the magnetic case.

Thus, only "large" BHs do exhibit a (classical) Attractor Mechanism, implemented through non-trivial (alias non-degenerate) critical points of the effective potential itself [6]. 


\subsection{4 "New Attractor" Approach}

Through the so-called "new attractor" approach [43], an equivalent form of the $n_{V}$ real criticality conditions (i.e. of the so-called Attractor Eqs.) for the various classes of critical points of $V$ can be obtained by plugging the criticality conditions themselves into the $n_{V}+1$ real RSG identities ${ }^{2}$ (3.4). By so doing, one respectively obtains:

- BPS Attractor Eqs.:

$$
q_{i}=\widehat{\lambda}_{i} Z
$$

While Eqs. (3.7) are $n_{V}$ real differential ones, the $n_{V}+1$ real Eqs. (3.14) are purely algebraic.

- Non-BPS Attractor Eqs.:

$$
q_{i}=\widehat{\lambda}_{i} Z-\frac{1}{2}\left(\frac{3}{2}\right)^{3 / 2} \frac{1}{Z} T^{x y z} Z_{y} Z_{z} \widehat{\lambda}_{i, x}
$$

\subsection{Derivatives of $T_{x y z}$}

Now, in order to proceed further, it is convenient to compute the Riemann-covariant derivative of the invariant tensor $T_{x y z}$, namely $T_{x y z ; w}$, a quantity which will be relevant in the subsequent treatment. By using the definition (2.9), one obtains

$$
T_{x y z ; w}=T_{(x y z) ; w}=-\sqrt{6}\left[-\frac{1}{2} g_{(y z} g_{x w)}+T_{r(y z} T_{x w) s} g^{r s}\right]=T_{(x y z ; w)} .
$$

Consequently, the condition 2.16) for the real special manifold $M_{5}$ to be a symmetric coset can be equivalently recast as follows (see e.g. page 14 of [19], and Eq. (3.2.1.9) of [20]):

$$
T_{r(y z} T_{x w) s} g^{r s}=\frac{1}{2} g_{(y z} g_{x w)} .
$$

One can then proceed further, and compute $T_{x y z ; w ; q}$. Starting from Eq. (3.16) one obtains (within the metric postulate)

$$
T_{x y z ; w ; q}=T_{(x y z ; w) ; q}=-2 \sqrt{6} T_{r(y z \mid ; q} T_{\mid x w) s} g^{r s}=-2 \sqrt{6} T_{r(y z ; q} T_{x w) s} g^{r s}=T_{(x y z ; w ; q)} .
$$

Through Eq. (3.16), this result can be further elaborated to give:

$$
T_{x y z ; w ; q}=12\left[-\frac{1}{2} g_{(y z} T_{x w q)}+T_{(q \mid v r} T_{p \mid y z} T_{x w) s} g^{p v} g^{r s}\right] .
$$

One can now introduce the following rank-5 completely symmetric tensor $\widetilde{E}_{x y z w q}$, which is the "RSG analogue" of the so-called $E$-tensor ${ }^{3}$ of SKG:

$$
\widetilde{E}_{x y z w q} \equiv \frac{1}{12} T_{x y z ; w ; q}=\frac{1}{12} T_{(x y z ; w ; q)}=\widetilde{E}_{(x y z w q)},
$$

\footnotetext{
${ }^{2}$ The extra real degree of freedom is only apparent, and removed by the homogeneity of degree one of the RSG identities 3.4 under a real overall shift of charges$$
q_{i} \longrightarrow \eta q_{i}, \eta \in \mathbb{R}
$$

${ }^{3}$ The $E$-tensor of SKG was firstly introduced in 32 , and it has been recently considered in the theory of extremal $d=4 \mathrm{BH}$ attractors in [44, 45, 39, 21, 40].
} 
satisfying by definition the relation

$$
T_{(q \mid v r} T_{p \mid y z} T_{x w) s} g^{p v} g^{r s}=\frac{1}{2} g_{(y z} T_{x w q)}+\widetilde{E}_{x y z w q},
$$

holding globally in RSG.

By recalling the symmetricity condition (2.16), Eqs. (3.18)-(3.21) yield

$$
T_{x y z ; w}=0 \Rightarrow T_{x y z ; w ; q}=0 \Leftrightarrow \widetilde{E}_{x y z w q}=0 \Leftrightarrow T_{(q \mid v r} T_{p \mid y z} T_{x w) s} g^{p v} g^{r s}=\frac{1}{2} g_{(y z} T_{x w q)} .
$$

\subsection{Generic RSG}

Let us now consider the value of the effective potential $V$ at the various classes of its critical points. By recalling its very definition (3.1), Eqs. (3.7) and (3.10) yield the following results:

\subsubsection{BPS}

Recall Eq. 3.8):

$$
V=Z^{2}
$$

Through Eq. (3.12, , this yields to

$$
\frac{S_{B H, d=5}}{\pi}=\frac{A_{H}}{4 \pi} \equiv R_{H}^{2}=V^{3 / 4}=|Z|^{3 / 2} .
$$

\subsubsection{Non-BPS and the "Dressed" Charges' Sum Rule}

$$
V=Z^{2}+\frac{3}{2} g^{x y} Z_{x} Z_{y}=Z^{2}+\frac{3}{8} \frac{1}{Z^{2}} g^{x y} T_{x z t} T_{w s y} Z^{z} Z^{t} Z^{w} Z^{s} .
$$

By recalling Eq. (3.16), the second term in the r.h.s. of Eq. (7.17) can be further elaborated as follows:

$$
Z_{x} Z^{x}=-\frac{1}{8} \sqrt{\frac{3}{2}} \frac{1}{Z^{2}} T_{(z t w ; s)} Z^{z} Z^{t} Z^{w} Z^{s}+\frac{3}{16} \frac{1}{Z^{2}}\left(Z_{x} Z^{x}\right)^{2}
$$

yielding $\left(Z_{x} Z^{x} \neq 0\right)$ :

$$
\frac{3}{2} Z_{x} Z^{x}=8 Z^{2}+\sqrt{\frac{3}{2}} \frac{T_{(x y z ; w)} Z^{x} Z^{y} Z^{z} Z^{w}}{Z_{u} Z^{u}} .
$$

Consequently at non-BPS $Z \neq 0$ critical points of $V$ it generally holds that:

$$
V=9 Z^{2}+\widetilde{\Delta}
$$

where the real quantity

$$
\widetilde{\Delta} \equiv \sqrt{\frac{3}{2}} \frac{T_{(x y z ; w)} Z^{x} Z^{y} Z^{z} Z^{w}}{Z_{u} Z^{u}}
$$

has been introduced. This latter is the "RSG analogue" of the complex quantity $\Delta$ introduced in SKG [44] (see also [45, 39, 21, 40]). As $\Delta$ enters the "dressed" charges' sum rule at non-BPS $(Z \neq 0)$ critical points of $V_{B H}$ in SKG (see e.g. Eqs. (282)-(284) of [44]), so $\widetilde{\Delta}$ enters the "dressed" charges' sum rule (3.28) at non-BPS critical points of $V$ in RSG, which further simplifies to (3.11) at least in symmetric RSG (having $\widetilde{\Delta}=0$ globally). Notice that, through Eq. (3.27) and definition 
(3.29), the (assumed) strictly positive definiteness of $g_{x y}$ (throughout all $M_{5}$, and in particular at the considered class of critical points of $V$ itself) yields

$$
Z^{2}+\frac{\widetilde{\Delta}}{8}>0
$$

Through Eq. (3.12), Eq. (3.28) yields

$$
\frac{S_{B H, d=5}}{\pi}=\frac{A_{H}}{4 \pi} \equiv R_{H}^{2}=V^{3 / 4}=\left(9 Z^{2}+\widetilde{\Delta}\right)^{3 / 4} .
$$

\subsubsection{The Functional $\widehat{\mathcal{I}}_{3}$}

Within a generic RSG, let us now consider the function

$$
\widehat{\mathcal{I}}_{3} \equiv \frac{1}{6} Z^{3}-\frac{3}{8} Z Z_{x} Z^{x}-\frac{1}{4} \sqrt{\frac{3}{2}} T_{x y z} Z^{x} Z^{y} Z^{z} .
$$

In general, $\widehat{\mathcal{I}}_{3}$ is a diffeomorphism- and symplectic- invariant function of the scalars $\phi^{x}$ in $M_{5}$, or equivalently a functional of the "dressed" charges $\left\{Z, Z_{x}\right\}$ in $M_{5}$. Its derivative reads (recalling Eq. (3.16) )

$$
\begin{aligned}
\widehat{\mathcal{I}}_{3, w} & =\widehat{\mathcal{I}}_{3 ; w}=-\sqrt{\frac{3}{2}} T_{x y z ; w} Z^{x} Z^{y} Z^{z} \\
& =-\frac{1}{2} Z_{x} Z^{x} Z_{w}+\frac{1}{3} g^{r s}\left(T_{r z y} T_{x w s}+T_{r z x} T_{y w s}+T_{r z w} T_{x y s}\right) Z^{x} Z^{y} Z^{z} .
\end{aligned}
$$

From the definition (3.29), it thus follows that

$$
\widetilde{\Delta}=-\frac{\widehat{\mathcal{I}}_{3, x} Z^{x}}{Z_{y} Z^{y}}
$$

The computation of $\widehat{\mathcal{I}}_{3}$ and $\widehat{\mathcal{I}}_{3, x}$ (respectively given by Eqs. 3.32 and 3.33 ) at the various classes of critical points of $V$ (specified by Eqs. (3.7)-(3.10) ) respectively yield to the following results.

\section{BPS}

$$
\begin{aligned}
\widehat{\mathcal{I}}_{3} & =\frac{1}{6} Z^{3} ; \\
\widehat{\mathcal{I}}_{3, x} & =0 .
\end{aligned}
$$

Thus, by recalling Eqs. (3.23) and (3.24), it follows that

$$
\frac{S_{B H, d=5}}{\pi}=\frac{A_{H}}{4 \pi} \equiv R_{H}^{2}=|Z|^{3 / 2}=V^{3 / 4}=\sqrt{6}\left|\widehat{\mathcal{I}}_{3}\right|^{1 / 2} .
$$


Non-BPS Eq. (3.27) and definition (3.29) yield

$$
Z_{x} Z^{x}=\frac{16}{3} Z^{2}+\frac{2}{3} \widetilde{\Delta}
$$

On the other hand, by recalling Eqs. (3.16) and (3.10), the term $T_{x y z} Z^{x} Z^{y} Z^{z}$ can be further elaborated at non-BPS $Z \neq 0$ critical points of $V$ as follows:

$$
T_{x y z} Z^{x} Z^{y} Z^{z}=-\frac{1}{2 \sqrt{6}} \frac{\left(Z_{x} Z^{x}\right)}{Z}\left(\widetilde{\Delta}-\frac{3}{2} Z_{y} Z^{y}\right) .
$$

Thus, definition 3.32 yields the following expression of $\widehat{\mathcal{I}}_{3}$ at non-BPS $Z \neq 0$ critical points of $V$ :

$$
\widehat{\mathcal{I}}_{3}=-\frac{9}{2} Z^{3}\left(1+\frac{7}{6} \frac{\widetilde{\Delta}}{3^{2} Z^{2}}\right) \Leftrightarrow \frac{\widetilde{\Delta}}{3^{2} Z^{2}}=-\frac{6}{7}\left(\frac{2}{9} \frac{\widehat{\mathcal{I}}_{3}}{Z^{3}}+1\right) .
$$

Thus, by recalling Eqs. (3.28) and (3.31), it follows that

$$
\frac{S_{B H, d=5}}{\pi}=\frac{A_{H}}{4 \pi} \equiv R_{H}^{2}=\left(9 Z^{2}+\widetilde{\Delta}\right)^{3 / 4}=V^{3 / 4}=\frac{3^{3 / 2}}{7^{3 / 4}}|Z|^{3 / 2}\left(1-\frac{4}{3} \frac{\widehat{\mathcal{I}}_{3}}{Z^{3}}\right)^{3 / 4},
$$

thus necessarily yielding

$$
\frac{3}{4}>\frac{\widehat{\mathcal{I}}_{3}}{Z^{3}}
$$

\subsection{Symmetric RSG and "Large" Charge Orbits}

Let us now consider the case in which 4

$$
M_{5}=\frac{G_{5}}{H_{5}}=\frac{G_{5}}{m c s\left(H_{5}\right)}
$$

is a symmetric coset.

(At least) in this case, $d_{i j k}$ is the unique $G_{5}$-invariant rank- 3 completely symmetric tensor, whereas $T_{x y z}$ is the unique $H_{5}$-invariant rank-3 completely symmetric tensor.

"Magic" symmetric $M_{5}$ 's are reported in Table 1 (see e.g. [32, and Refs. therein; see also [46] for a brief review and list of Refs.).

Besides these four isolated cases, there are two infinite sequences of other symmetric real special manifolds, namely the so-called Jordan symmetric sequence

$$
M_{J, 5, n} \equiv S O(1,1) \times \frac{S O(1, n)}{S O(n)}, n=n_{V}-1 \in \mathbb{N},
$$

and the non-Jordan symmetric sequence 41

$$
M_{n J, 5, n} \equiv \frac{S O(1, n)}{S O(n)}, n=n_{V} \in \mathbb{N},
$$

\footnotetext{
4 "mcs" is acronym for maximal compact subgroup (with symmetric embedding). Unless otherwise noted, all considered embeddings are symmetric. Moreover, the subscript "max" denotes the maximality of the embedding throughout.
} 


\begin{tabular}{|c||c|c|c|}
\hline$J_{3}^{\mathbb{A}}$ & $\begin{array}{c}M_{5}=\frac{G_{5}}{H_{5}}=\frac{S \operatorname{Str}_{0}\left(J_{3}\right)}{\text { Aut }\left(J_{3}\right)} \\
=\begin{array}{c}\mathcal{O}_{B P S, \text { large }} \\
r=3\end{array}\end{array}$ & $\begin{array}{c}M_{5}^{*}=\frac{G_{5}}{\widetilde{H}_{5}} \\
=\begin{array}{c}\mathcal{O}_{n B P S, \text { large }} \\
H_{5} \equiv m c s\left(G_{5}\right)\end{array}\end{array}$ & $\widetilde{M}_{n B P S, \text { large }}=\frac{\widetilde{H}_{5}}{\widetilde{h}_{5}}$, \\
\hline$J_{3}^{\mathbb{Q}}, q=8$ & $\frac{E_{6(-26)}}{F_{4(-52)}}$ & $\frac{E_{6(-26)}}{F_{4(-20)}}$ & $\frac{F_{4(-20)}}{S O(9)}$ \\
\hline$J_{3}^{\mathbb{H}}, q=4$ & $\frac{S U^{*}(6)}{U S p(6)}$ & $\frac{S U^{*}(6)}{U S p(4,2)}$ & $\frac{U S p(4,2)}{U S p(4) \times U S p(2)}$ \\
\hline$J_{3}^{\mathbb{C}}, q=2$ & $\frac{S L(3, \mathbb{C})}{S U(3)}$ & $\frac{S L(3, \mathbb{C})}{S U(2,1)}$ & $\frac{S U(2,1)}{S U(2) \times U(1)}$ \\
\hline$J_{3}^{\mathbb{R}}, q=1$ & $\frac{S L(3, \mathbb{R})}{S O(3)}$ & $\frac{S L(3, \mathbb{R})}{S O(2,1)}$ & $\frac{S O(2,1)}{S O(2)}$ \\
\hline
\end{tabular}

Table 1: Homogeneous symmetric real special vector multiplets' scalar manifolds $M_{5}$ of $\mathcal{N}=2, d=5$ "magic" supergravity. $M_{5}$ 's also are: 1$)$ the non-BPS $Z \neq 0$ moduli spaces of $\mathcal{N}=2, d=4$ special Kähler symmetric vector multiplets' scalar manifolds [23]; and 2) the "large" $\frac{1}{2}$-BPS charge orbits $\mathcal{O}_{B P S \text {,large' }}$ of $\mathcal{N}=2, d=5$ Maxwell-Einstein supergravity itself [19]. The "large"non-BPS $Z \neq 0$ charge orbits $\mathcal{O}_{n B P S \text {, large }}=M_{5}^{*}$ (see e.g. Table 5 of [48] and Refs. therein) and the related non-BPS $Z \neq 0$ moduli spaces $\mathcal{M}_{n B P S \text {, large }}$ are reported, as well. The rank $r$ of the orbit is defined as the minimal number of charges defining a representative solution. As observed in [23], for "magic" supergravities $n_{V}=\operatorname{dim}_{\mathbb{R}} M_{5}=3 q+2$, whereas $\operatorname{dim}_{\mathbb{R}} \mathcal{M}_{n B P S \text {,large }}=2 q$, and $\operatorname{Spin}(1+q) \subset \widetilde{h}_{5}$. See text for more details

$n_{V}$ being the number of Abelian vector supermultiplets coupled to the $\mathcal{N}=2, d=5$ supergravity one.

The sequence (3.45) is the only (sequence of) symmetric RSG which is not related to Jordan algebras of degree three. It is usually denoted by $L(-1, n-1)$ in the classification of homogeneous Riemannian $d$-spaces (see e.g. [32], and Refs. therein). It will not be further considered here, because it does not correspond to symmetric spaces in four dimensions.

$G_{5}$ and $H_{5}$ can respectively be interpreted as the reduced structure group Str $_{0}$ and the automorphism group Aut of the corresponding Euclidean Jordan algebra of degree three (see e.g. [47. for a recent review, and Refs. therein):

$$
M_{5}=\frac{G_{5}}{H_{5}}=\frac{\operatorname{Str}_{0}\left(J_{3}\right)}{\operatorname{Aut}\left(J_{3}\right)} .
$$


Furthermore, (at leas $\left.t^{5}\right)$ in symmetric RSG, due to Eqs. 2.16) and $(3.33)$, it holds that

$$
\widehat{\mathcal{I}}_{3, x}=\widehat{\mathcal{I}}_{3 ; x}=0 .
$$

In other words, $\widehat{\mathcal{I}}_{3}$ is independent on all scalars $\phi^{x}$. Furthermore:

$$
\widehat{\mathcal{I}}_{3}=\mathcal{I}_{3},
$$

where $\mathcal{I}_{3}$ is the unique cubic invariant of the relevant electric (ir)repr. $\mathbf{R}_{Q}$ of $d=5 U$-duality $G_{5}$, defined by 7.2 . As mentioned above, $d_{i j k}$ is $G_{5}$-invariant in all RSG, whereas $d^{i j k}$ is $G_{5}$-invariant at least in symmetric RSG.

In this framework, by virtue of the relations (7.27) or (7.31), the Bekenstein-Hawking entropyarea formula (3.12) can be completed as follows (recall Eq. (3.48)):

$$
\frac{S_{B H, d=5}}{\pi}=\frac{A_{H}}{4 \pi} \equiv R_{H}^{2}=\left(\left.V\right|_{\partial V=0}\right)^{3 / 4}=\sqrt{6}\left|\mathcal{I}_{3}\right|^{1 / 2}=\sqrt{6}\left|\widehat{\mathcal{I}}_{3}\right|^{1 / 2} .
$$

Furthermore, in RSG based on symmetric cosets $\frac{G_{5}}{H_{5}}$ the representation space of the irrepr. of $G_{5}$ in which the (electric or magnetic) charges sit admit a stratification in disjoint orbits [15, 19]. Such orbits are homogeneous, in some case symmetric, manifolds.

The charge orbits supporting non-degenerate (in the sense specified above; see the end of Subsect. 3.1) critical points of $V$ are called "large" orbits, because they correspond to the previously introduced class of "large" BHs with non-vanishing Bekenstein-Hawking entropy-area (see Eq. (3.12)). On the other hand, charge orbits corresponding to "small" BHs (having vanishing Bekenstein-Hawking entropy-area) are correspondingly dubbed "small" orbits.

In the treatment of symmetric RSG performed in present Subsection, only "large" orbits, firstly found in [19, are considered.

In Sect. 4 , through the properties of the function $\widehat{\mathcal{I}}_{3}$ defined by Eq. 3.32 , the stabilizers of all "small" charge orbits of symmetric RSG will be derived, by suitably solving $G_{5}$-invariant (sets of) defining differential constraints, as well as by performing suitable group theoretical procedures.

We can now specialize the results obtained in Subsect. 3.3 and in Subsubsect. 3.3 .3 to "magic" symmetric RSG. The detailed treatment of $\mathcal{N}=2$ Jordan symmetric sequence (3.44) will be given in Sect. 6. Actually, the "large" charge orbits of (3.44) have been already considered in [19] (see also [23] for the study of corresponding moduli spaces), but in Sect. 6 the treatment is further refined.

\subsubsection{BPS}

Eqs. (3.35) and (3.48) yield to

$$
\widehat{\mathcal{I}}_{3}=\frac{1}{6} Z^{3}=\mathcal{I}_{3}
$$

and thus:

$$
\frac{S_{B H, d=5}}{\pi}=\frac{A_{H}}{4 \pi} \equiv R_{H}^{2}=\left(\left.V\right|_{\partial V=0}\right)^{3 / 4}=\sqrt{6}\left|\mathcal{I}_{3}\right|^{1 / 2}=\sqrt{6}\left|\widehat{\mathcal{I}}_{3}\right|^{1 / 2}=|Z|^{3 / 2} .
$$

${ }^{5}$ Notice that, from Eq. 3.33 , it follows that

$$
\widehat{\mathcal{I}}_{3, w}=0 \Leftrightarrow T_{x y z ; w} Z^{x} Z^{y} Z^{z}=0,
$$

whose 2.16 is a solution. 
Such a "large" BH is supported by (electric) charges belonging to the "large" charge orbit (homogeneous symmetric manifold) [19]

$$
\mathcal{O}_{B P S, \text { large }}=\frac{G_{5}}{H_{5}}=M_{5}
$$

The compactness of $H_{5}$ yields the absence of a moduli space related to $\frac{1}{2}$-BPS "large" attractor solutions, a fact that can be seen also from the expression of the Hessian matrix of $V$ evaluated along the BPS criticality constraints (3.7) (see Eq. (3.72) below).

It is worth remarking that $M_{5}$ 's also are the non-BPS $Z \neq 0$ moduli spaces of $\mathcal{N}=2, d=4$ special Kähler symmetric vector multiplets' scalar manifolds [23].

Notice that in general

$$
\operatorname{dim}_{\mathbb{R}} M_{5}=n_{V}
$$

As observed in [23], for "magic" supergravities (based on Euclidean Jordan algebras of degree three $J_{3}^{\mathbb{A}}$ over the division algebras $\mathbb{A}$ ) it holds:

$$
\begin{aligned}
& \operatorname{dim}_{\mathbb{R}} M_{5}=3 q+2, \\
& q \equiv \operatorname{dim}_{\mathbb{R}}(\mathbb{A}=\mathbb{O}, \mathbb{H}, \mathbb{C}, \mathbb{R})=(8,4,2,1) .
\end{aligned}
$$

\subsubsection{Non-BPS}

Eqs. (3.40) and (3.48) yield to

$$
\widehat{\mathcal{I}}_{3}=-\frac{9}{2} Z^{3}=\mathcal{I}_{3}
$$

Indeed, from its very definition, in this framework it globally holds that

$$
\widetilde{\Delta}=0,
$$

and thus (recall Eq. (3.11)):

$$
\frac{3}{2} Z_{x} Z^{x}=8 Z^{2} \Leftrightarrow V=9 Z^{2}
$$

Through Eq. (3.49), it thus follows that

$$
\frac{S_{B H, d=5}}{\pi}=\frac{A_{H}}{4 \pi} \equiv R_{H}^{2}=\left(\left.V\right|_{\partial V=0}\right)^{3 / 4}=\sqrt{6}\left|\mathcal{I}_{3}\right|^{1 / 2}=\sqrt{6}\left|\widehat{\mathcal{I}}_{3}\right|^{1 / 2}=3^{3 / 2}|Z|^{3 / 2} .
$$

Such a "large" BH is supported by (electric) charges belonging to the "large" charge orbit (homogeneous symmetric manifold)

$$
\mathcal{O}_{n B P S, \text { large }}=\frac{G_{5}}{\widetilde{H}_{5}}=M_{5}^{*}
$$

where $\widetilde{H}_{5}$ is the unique non-compact, real form of $H_{5}=m c s\left(G_{5}\right)$ which admits a maximal symmetric embedding into $G_{5}$ :

$$
G_{5} \supsetneq \max \widetilde{H}_{5} .
$$

The homogeneous symmetric pseudo-Riemannian manifold $M_{5}^{*}$ is the "*-version" of $M_{5}$, obtained through timelike $d=6 \rightarrow 5$ reduction from the corresponding anomaly-free uplifted $\mathcal{N}=(1,0)$, $d=6$ chiral theory (see e.g. Table 5 of [48], and Refs. therein). Notice that Eq. (3.59) yields to

$$
\mathcal{O}_{n B P S, \text { large }}=\mathcal{O}_{B P S, \text { large }}^{*}
$$


in the sense we have just specified.

The non-compactness of $\widetilde{H}_{5}$ implies the existence of a non-BPS moduli space [23]

$$
\mathcal{M}_{n B P S, \text { large }} \equiv \frac{\widetilde{H}_{5}}{m c s\left(\widetilde{H}_{5}\right)} \equiv \frac{\widetilde{H}_{5}}{\widetilde{h}_{5}} .
$$

As observed in [23], for "magic" supergravities it holds (see e.g. also Table 8 of [46], and Refs. therein):

$$
\begin{aligned}
& \operatorname{dim}_{\mathbb{R}} \mathcal{M}_{n B P S, \text { large }}=2 q ; \\
& \operatorname{Spin}(1+q) \subset \widetilde{h}_{5},
\end{aligned}
$$

where $\operatorname{Spin}(1+q)$ is the spin group in $1+q$ dimensions. Notice that $2 q$ is the number of $d=6$ (scalarless) vector multiplets needed for an anomaly-free uplift of the considered $\mathcal{N}=2, d=5$ "magic" Maxwell-Einstein supergravity to the corresponding $\mathcal{N}=(1,0)$ chiral quarter-minimal "magic" supergravity in $d=6$ (see e.g. Sect. 5 of [20], and Refs. therein). is

Thus, by recalling (3.54), the number $\sharp$ of "non-flat" scalar degrees of freedom along $\mathcal{O}_{n B P S \text {,large }}$

$$
\sharp_{n B P S, \text { large }} \equiv \operatorname{dim}_{\mathbb{R}} M_{5}-\operatorname{dim}_{\mathbb{R}} \mathcal{M}_{n B P S, \text { large }}=q+2 .
$$

The "large" non-BPS $Z \neq 0$ charge orbits $\mathcal{O}_{n B P S \text {,large }}=M_{5}^{*}$, and the related non-BPS $Z \neq 0$

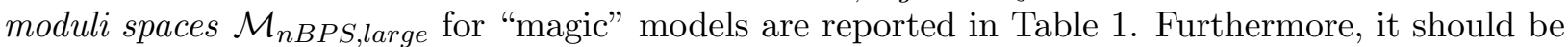
recalled that the Jordan symmetric sequence (3.44) is related to the reducible rank-3 Euclidean Jordan algebra $\mathbb{R} \oplus \boldsymbol{\Gamma}_{1, n}$, where $\boldsymbol{\Gamma}_{1, n}$ is the rank-2 Jordan algebra with a quadratic form of Lorentzian signature $(1, n)$, i.e. the Clifford algebra of $O(n, 1)$ [49].

\subsection{Hessian Matrix of $V$}

From its very definition (3.1), the first derivative of $V$ reads (recall Eq. (3.6)

$$
V_{x} \equiv V_{, x}=V_{; x}=2\left(2 Z Z_{x}-\sqrt{\frac{3}{2}} T_{x y z} g^{y s} g^{z t} Z_{s} Z_{t}\right) .
$$

By further differentiating, the global expression of the real Hessian $n_{V} \times n_{V}$ matrix of $V$ in a generic RSG can be computed as follows:

$$
\begin{aligned}
V_{x ; y}= & V_{; x ; y} \\
= & \frac{8}{3} g_{x y}\left(Z^{2}-\frac{3}{8} Z_{w} Z^{w}\right)+2 Z_{x} Z_{y}-8 \sqrt{\frac{2}{3}} Z T_{x y z} Z^{z}+ \\
& +2\left(T_{x y s} T_{r z w}+4 T_{x z r} T_{y w s}\right) g^{r s} Z^{z} Z^{w} \\
= & V_{(x ; y)},
\end{aligned}
$$

where Eqs. (3.5) and (3.16) have been used.

On the other hand, by recalling definition $(3.20)$ and Eq. (3.33), it can be computed that

$$
\widehat{\mathcal{I}}_{3 ; x ; y}=-3 \sqrt{\frac{3}{2}}\left(4 \widetilde{E}_{x y z w r} Z^{r}+\frac{2}{3} Z T_{x y z ; w}-\sqrt{\frac{2}{3}} T_{z w s ; x} T_{y s^{\prime} r} g^{s s^{\prime}} Z^{r}\right) Z^{z} Z^{w} .
$$


Then, further elaboration of Eq. (3.66) is possible for $Z \neq 0$. Indeed, in such a case Eq. (3.67) implies that (recall Eq. (3.16)

$$
\begin{aligned}
& T_{x z w ; y} Z^{z} Z^{w} \\
= & -\frac{1}{\sqrt{6}} \frac{1}{Z} \widehat{\mathcal{I}}_{3 ; x ; y}-\frac{6}{Z} \widetilde{E}_{x y z w r} Z^{z} Z^{w} Z^{r} \\
& +\frac{1}{2 Z}\left(Z_{w} Z^{w}\right) T_{x y z} Z^{z}+\frac{1}{Z} Z_{y} T_{x z w} Z^{z} Z^{w} \\
& -\frac{1}{Z}\left(T_{x r p} T_{y r^{\prime} s} T_{t z w}+2 T_{x w p} T_{y r^{\prime} s} T_{t z r}\right) g^{r r^{\prime}} g^{t p} Z^{s} Z^{z} Z^{w} .
\end{aligned}
$$

Notice that the symmetry properties $\widehat{\mathcal{I}}_{3 ; x ; y}=\widehat{\mathcal{I}}_{3 ;(x ; y)}$ and $T_{x z w ; y} Z^{z} Z^{w}=T_{(x z w ; y)} Z^{z} Z^{w}$ are not manifest respectively from Eqs. (3.67) and 3.68 , due to the presence of $\widetilde{E}_{x y z w r}, T_{x y z ; w}$, and $\widehat{\mathcal{I}}_{3 ; x ; y}$ itself. By plugging Eq. (3.68) back into Eq. (3.66), the following result is achieved:

$$
\begin{aligned}
V_{x ; y}= & V_{; x ; y} \\
= & 4 Z_{x} Z_{y}+\frac{8}{3} Z^{2} g_{x y}-8 \sqrt{\frac{2}{3}} Z T_{x y z} Z^{z} \\
& +\frac{1}{Z} \widehat{\mathcal{I}}_{3 ; x ; y}+\frac{6 \sqrt{6}}{Z} \widetilde{E}_{x y z w r} Z^{z} Z^{w} Z^{r} \\
& -\sqrt{\frac{3}{2}} \frac{1}{Z}\left(Z_{w} Z^{w}\right) T_{x y z} Z^{z}-\frac{\sqrt{6}}{Z} Z_{y} T_{x z w} Z^{z} Z^{w} \\
& +\frac{\sqrt{6}}{Z}\left(T_{x r p} T_{y r^{\prime} s} T_{t z w}+2 T_{x w p} T_{y r^{\prime} s} T_{t z r}\right) g^{r r^{\prime}} g^{t p} Z^{s} Z^{z} Z^{w} \\
& +4 T_{x z w} T_{y s w^{\prime}} g^{w w^{\prime}} Z^{z} Z^{s},
\end{aligned}
$$

holding true for $Z \neq 0$. Once again, notice that the symmetry property $V_{x ; y}=V_{(x ; y)}$ is not manifest from Eq. 3.69, due to the presence of $\widetilde{E}_{x y z w r}$ and $\widehat{\mathcal{I}}_{3 ; x ; y}$.

By inserting the global condition (2.16) into Eq. (3.66), one obtains that

$$
V_{x ; y}=V_{; x ; y}=4 Z_{x} Z_{y}+\frac{8}{3} Z^{2} g_{x y}-8 \sqrt{\frac{2}{3}} Z T_{x y z} Z^{z}+4 T_{x z w} T_{y s w^{\prime}} g^{w w^{\prime}} Z^{z} Z^{s} \equiv V_{; x ; y, s y m m .} .
$$

This is the global expression of the real Hessian $n_{V} \times n_{V}$ matrix of $V$ (at least) in symmetric RSG, and indeed it matches the result given by Eq. (5-1) of [19] (see also [20]). Thus, Eqs. (3.66) and (3.70) yield to the following result:

$$
V_{; x ; y}=V_{; x ; y, s y m m .}-g_{x y}\left(Z_{w} Z^{w}\right)-2 Z_{x} Z_{y}+2\left(2 T_{x w z} T_{y s z^{\prime}}+T_{x y z} T_{s w z^{\prime}}\right) g^{z z^{\prime}} Z^{w} Z^{s} .
$$

\subsubsection{Evaluation at Critical Points of $V$}

We will now proceed to evaluate the Hessian matrix of $V$ given by Eq. (3.66) at the various classes of critical points of $V$ itself, as given by Eqs. (3.7)-(3.10).

BPS The necessary and sufficient BPS criticality constraints 3.7 plugged into Eq. 3.66 yield

$$
V_{; x ; y}=\frac{8}{3} g_{x y} Z^{2} .
$$


Eq. (3.72) holds for a generic RSG, and it matches the result given by Eq. (5-2) of [19]. For a strictly positive definite $g_{x y}$ (as it is usually assumed), it implies that the Hessian matrix of $V$ at its BPS critical points has all strictly positive eigenvalues.

As mentioned above, the lack of Hessian massless modes at $\frac{1}{2}$-BPS critical points of $V$ determines the absence of a moduli space in BPS attractor solutions, which thus have all scalar fields $\phi^{x}$ stabilized at the (unique) event horizon of the considered (electric) $d=5$ extremal BH.

Non-BPS It is here worth noticing that Eq. 3.10 yields to

$$
Z_{x} Z^{x}=\sqrt{\frac{3}{2}} \frac{1}{2 Z} T_{x y z} Z^{x} Z^{y} Z^{z} .
$$

By recalling the "dressed" charges' sum rule given by Eq. (3.27) and definition (3.29), Eq. (3.73) implies

$$
\frac{32}{3} Z^{2}+\widetilde{\Delta}=\sqrt{\frac{3}{2}} \frac{1}{Z} T_{x y z} Z^{x} Z^{y} Z^{z}
$$

On the other hand, by using Eq. (3.16), one can compute also that

$$
Z_{x} Z^{x}=-\frac{1}{8} \sqrt{\frac{3}{2}} \frac{1}{Z^{2}} T_{x y z ; w} Z^{x} Z^{y} Z^{z} Z^{w}+\frac{3}{16} \frac{1}{Z^{2}}\left(Z_{x} Z^{x}\right)^{2} .
$$

By dividing by $Z_{x} Z^{x} \neq 0$, one then obtains the "dressed" charges' sum rule given by Eq. (3.27). However, one can also interpret Eq. (3.75) as a quadratic Eq. in the unknown $Z_{x} Z^{x}$, obtaining the result

$$
0<Z_{x} Z^{x}=\frac{8}{3} Z^{2} \pm \sqrt{\frac{64}{9} Z^{4}-\frac{2}{3} \widehat{\mathcal{I}}_{3 ; x} Z^{x}} .
$$

When $\widehat{\mathcal{I}}_{3 ; x}=0$ (i.e. - at least - for symmetric RSG) Eq. (3.76) consistently yields [19]:

$$
\frac{3}{2} Z_{x} Z^{x}=8 Z^{2}
$$

\section{4 "Small" Charge Orbits and Moduli Spaces in Symmetric "Magic" RSG}

In the treatment of symmetric RSG performed in Subsect. 3.4, only "large" charge orbits, supporting solutions to the corresponding Attractor Eqs. (and firstly found in [19]; see also [20]), have been considered.

In the present Section, by exploiting the properties of the functional $\widehat{\mathcal{I}}_{3}$ introduced in Subsubsect. 3.3.3, all "small" charge orbits of "magic" symmetric RSG will be explicitly determined through the resolution of $G_{5}$-invariant defining (differential) constraints both in "bare" and "dressed" charges bases, as well as through group theoretical techniques.

By definition, $\widehat{\mathcal{I}}_{3}\left(=\mathcal{I}_{3}\right.$ in symmetric RSG, as discussed in Subsect. 3.4, see Eq. 3.48) vanishes for all "small" charge orbits. Consequently, such orbits do not support solutions to the Attractor Eqs. (alias criticality conditions of the effective potential $V$; see Eqs. (3.7)-(3.10), or Eqs. (3.14)-(3.15) in the so-called "new attractor" approach). In other words, the (classical) Attractor Mechanism does not hold for "small" charge orbits, which indeed do support BH states which are intrinsically quantum, in the sense that the effective description through Einstein supergravity fail for them. 
Besides the condition of vanishing $\widehat{\mathcal{I}}_{3}$, further conditions, formulated in terms of derivatives of $\widehat{\mathcal{I}}_{3}$ in some charge basis, may be needed to fully characterize the class of "small" orbits under consideration. It is here worth pointing out that the (sets of) $G_{5}$-invariant constraints which define "small" charge orbits in homogeneous symmetric real special manifolds $\frac{G_{5}}{H_{5}}$ are characterizing equations for charges (in both "bare" and "dressed" bases), but they actually are identities in all scalar fields $\phi^{x}$, and thus they hold globally in $\frac{G_{5}}{H_{5}}$. This is to be contrasted with "large" charge orbits, which are defined through the Attractor Eqs. themselves, which are at the same time characterizing equations for charges (in both "bare" and "dressed" bases) and stabilization equations for the scalars $\phi^{x}$ at the event horizon of the extremal $\mathrm{BH}$.

As it is well known [23], at non-BPS $Z \neq 0$ critical points of $V$ some scalars are actually unstabilized at the (unique) event horizon of the corresponding "large" extremal BH solutions. Such unstabilized $\phi^{x}$ 's span the moduli space $\mathcal{M}_{n B P S \text {,large }}$ (given by Eq. (3.62)), associated with an hidden compact symmetry of the non-BPS $Z \neq 0$ Attractor Eqs. themselves, which can be traced back to the non-compactness of the stabilizer of the non-BPS $Z \neq 0$ "large" charge orbit $\mathcal{O}_{n B P S, \text { large }}$ (see Eq. (3.59), to be contrasted with Eq. (3.52)).

The "small" charge orbits are homogeneous manifolds of the form:

$$
\mathcal{O}_{\text {small }}=\frac{G_{5}}{\mathcal{S}_{\max } \rtimes \mathcal{T}},
$$

where $\rtimes$ denotes semi-direct group product throughout, and $\mathcal{T}$ is the non-semi-simple part of the stabilizer of $\mathcal{O}_{\text {small }}$, which in all symmetric RSG (with some extra features characterizing the symmetric Jordan sequence, see Sect. 6) can be identified with an Abelian translational subgroup of $G_{5}$ itself.

One can associate a moduli space also to "small" charge orbits, by observing that the noncompactness of $\mathcal{S}_{\max } \rtimes \mathcal{T}$ yields the existence of a corresponding moduli space defined a: 6

$$
\mathcal{M}_{\text {small }} \equiv \frac{\mathcal{S}_{\max }}{\operatorname{mcs}\left(\mathcal{S}_{\max }\right)} \rtimes \mathcal{T}
$$

Note that, differently from "large" orbits, for "small" orbits there exists a moduli space $\mathcal{M}_{\text {small }}=\mathcal{T}$ also when $\mathcal{S}_{\max }$ is compact. As found in [50, 51] for "large" charge orbits of $\mathcal{N}=2, d=4$ stu model, and recently proved in a model-independent way in [52, the moduli spaces of charge orbits are defined all along the scalar flows, and thus they can be interpreted as moduli spaces of unstabilized scalars at the event horizon (if any) of the extremal $\mathrm{BH}$, as well as moduli spaces of the ADM mass of the extremal $\mathrm{BH}$ at spatial infinity. In the "small" case, the interpretation at the event horizon breaks down, simply because such an horizon does not exist at all (at least in Einsteinian supergravity approximation).

In general, the number $\sharp$ of "non-flat" scalar degrees of freedom supported by a ("large" or "small") charge orbit $\mathcal{O}$ with associated moduli space $\mathcal{M}$ is defined as follows:

$$
\sharp \equiv \operatorname{dim}_{\mathbb{R}} M_{d=5}-\operatorname{dim}_{\mathbb{R}} \mathcal{M} .
$$

As an example, let us briefly consider the maximal $\mathcal{N}=8, d=5$ supergravity, whose "large" and "small" charge orbits have been classified in [15]. The scalar manifold of the theory is

$$
M_{\mathcal{N}=8, d=5}=\frac{E_{6(6)}}{U S p(8)}, \operatorname{dim}_{\mathbb{R}}=42 .
$$

\footnotetext{
${ }^{6}$ We thank M. Trigiante for a discussion on the "flat" directions of "small" charge orbits.
} 
1. The unique "large" charge orbit is $\frac{1}{8}$-BPS:

$$
\mathcal{O}_{\frac{1}{8}-B P S}=\frac{E_{6(6)}}{F_{4(4)}}, \operatorname{dim}_{\mathbb{R}}=26
$$

with corresponding moduli space 23

$$
\mathcal{M}_{\frac{1}{8}-B P S}=\frac{F_{4(4)}}{U S p(6) \times U S p(2)}, \operatorname{dim}_{\mathbb{R}}=28 .
$$

Thus, the number of "non-flat" directions along $\mathcal{O}_{\frac{1}{8}-B P S}$ reads

$$
\sharp_{\frac{1}{8}-B P S} \equiv \operatorname{dim}_{\mathbb{R}} M_{\mathcal{N}=8, d=5}-\operatorname{dim}_{\mathbb{R}} \mathcal{M}_{\frac{1}{8}-B P S}=14 .
$$

Since the charge orbit is "large", $\sharp_{\frac{1}{8}-B P S}$ also expresses the actual number of scalar degrees of freedom which are stabilized in terms of the electric (magnetic) charges in the near-horizon geometry of the extremal black hole (black string) under consideration.

2. The "small" $\frac{1}{4}$-BPS orbit is

$$
\mathcal{O}_{\frac{1}{4}-B P S}=\frac{E_{6(6)}}{S O(5,4) \rtimes \mathbb{R}^{16}}, \operatorname{dim}_{\mathbb{R}}=26,
$$

with corresponding moduli space

$$
\mathcal{M}_{\frac{1}{4}-B P S}=\frac{S O(5,4)}{S O(5) \times S O(4)} \rtimes \mathbb{R}^{16}, \operatorname{dim}_{\mathbb{R}}=36 .
$$

Thus, the number of "non-flat" directions along $\mathcal{O}_{\frac{1}{4}-B P S}$ reads

$$
\sharp_{\frac{1}{4}-B P S} \equiv \operatorname{dim}_{\mathbb{R}} M_{\mathcal{N}=8, d=5}-\mathcal{M}_{\frac{1}{4}-B P S}=6 .
$$

3. The "small" $\frac{1}{2}$-BPS orbit is

$$
\mathcal{O}_{\frac{1}{2}-B P S}=\frac{E_{6(6)}}{S O(5,5) \rtimes \mathbb{R}^{16}}, \operatorname{dim}_{\mathbb{R}}=17,
$$

with corresponding moduli space

$$
\mathcal{M}_{\frac{1}{2}-B P S}=\frac{S O(5,5)}{S O(5) \times S O(5)} \rtimes \mathbb{R}^{16}=M_{(2,2), d=6} \rtimes \mathbb{R}^{16}, \operatorname{dim}_{\mathbb{R}}=41,
$$

where $M_{(2,2), d=6}$ is the scalar manifold of maximal (non-chiral) supergravity in $d=6$. Thus, the number of "non-flat" directions along $\mathcal{O}_{\frac{1}{2}-B P S}$ reads

$$
\sharp_{\frac{1}{2}-B P S} \equiv \operatorname{dim}_{\mathbb{R}} M_{\mathcal{N}=8, d=5}-\mathcal{M}_{\frac{1}{2}-B P S}=1 \text {. }
$$

As we will point out more than once in the treatment below, result 4.13) expresses the pretty general fact that the unique "non-flat" direction along maximally supersymmetric (namely, $\frac{1}{2}$-BPS) charge orbits is the Kaluza-Klein radius in the dimensional reduction $d=5 \rightarrow d=4$. 


\begin{tabular}{|c||c|c|}
\hline$q$ & $Q_{q}$ & $\mathcal{A}_{q}$ \\
\hline \hline 8 & - & - \\
\hline 4 & 2 & $S O(3)$ \\
\hline 2 & 2 & $S O(2)$ \\
\hline 1 & - & - \\
\hline
\end{tabular}

Table 2: $Q_{q}$ and $\mathcal{A}_{q}$ for the various $\mathcal{N}=2, d=5$ "magic" supergravities (based on $J_{3}^{\mathbb{A}}$, $\mathbb{A}=\mathbb{O}, \mathbb{H}, \mathbb{C}, \mathbb{R})$, classified by $q \equiv \operatorname{dim}_{\mathbb{R}} \mathbb{A}=8,4,2,1$

In the treatment of Subsect. 4.1, the $G_{5}$-invariant constraints defining all classes of "small" charge orbits in all symmetric RSG will be derived. Then they will be solved both in "bare" and "dressed" charge bases in Subsect. A. Furthermore, in App. B the origin of "small" charge orbits (and in particular of $\mathcal{T}$ ) will be elucidated through group theoretical procedures (namely, İönü-Wigner contractions [57, 58] and $S O(1,1)$-three grading).

While the treatment of Subsect. 4.1 holds for all symmetric RSG, the treatments given in Apps. $\mathrm{A}$ and B strictly fit only the isolated cases of symmetric RSG provided by the so-called "magic" symmetric RSG's [28, 29, 30, 31]. The main results of Apps. A and B are reported in Tables 3 and 4 (the symmetric Jordan sequence (3.44) is considered in Sect. 6). In the "magic" octonionic case $J_{3}^{\mathbb{Q}}(q=8)$, the results of [15] are matched.

Below we summarize the main results of Apps. $\mathrm{A}$ and $\mathrm{B}$.

- The "small" lightlike BPS charge orbit $\left(\operatorname{dim}_{\mathbb{R}}=3 q+2\right)$

$$
\mathcal{O}_{\text {lightlike }, B P S}=\frac{G_{5}}{\left(S O(q+1) \times \mathcal{A}_{q}\right) \rtimes \mathbb{R}^{\left(\operatorname{spin}(q+1), \operatorname{spin}\left(Q_{q}\right)\right)}},
$$

thus with

$$
\begin{aligned}
\mathcal{S}_{\text {max }, \text { lightlike }, B P S} & =S O(q+1) \times \mathcal{A}_{q} \\
\mathcal{T}_{\text {lightlike }, B P S} & =\mathbb{R}^{\left(\operatorname{spin}(q+1), \operatorname{spin}\left(Q_{q}\right)\right)} .
\end{aligned}
$$

$Q_{q}$ and $\mathcal{A}_{q}$, a further factor group in $\mathcal{S}_{\text {max }}$, are given by Table 2. Furthermore, we define

$$
\begin{aligned}
\operatorname{spin}(q+1) & \equiv \operatorname{dim}_{\mathbb{R}}(\operatorname{Spin}(q+1)) \\
\operatorname{spin}\left(Q_{q}\right) & \equiv \operatorname{dim}_{\mathbb{R}}\left(\operatorname{Spin}\left(Q_{q}\right)\right),
\end{aligned}
$$

with $\operatorname{Spin}(q+1)$ and $\operatorname{Spin}\left(Q_{q}\right)$ respectively denoting the spinor irreprs. in $q+1$ and $Q_{q}$ dimensions. It is worth remarking that $\mathcal{A}_{q}$ is independent on the space-time dimension $(d=$ $3,4,5,6)$ in which the quarter-minimal symmetric "magic" (Maxwell-Einstein) supergravity (classified by $q=8,4,2,1)$ is considered. It also holds that

$$
\begin{aligned}
d & =5,6: \widehat{G}_{c e n t}=S O(1,1) \times S O(q-1) \times \mathcal{A}_{q} ; \\
d & =3,4: \widehat{G}_{c e n t}=G_{\text {paint }}=S O(q) \times \mathcal{A}_{q},
\end{aligned}
$$

where the groups $\widehat{G}_{c e n t}$ and $G_{\text {paint }}$ are usually introduced in the treatment of supergravity billiards and timelike reductions (for recent treatment and set of related Refs., see e.g. [48; 
see also Table 5 therein, also for subtleties concerning the case $q=8$ in $d=5,6$ ). The moduli space corresponding to (4.14) is purely translational:

$$
\mathcal{M}_{\text {lightlike,BPS }}=\mathbb{R}^{\left(\operatorname{spin}(q+1), \operatorname{spin}\left(Q_{q}\right)\right)},
$$

with real dimension

$$
\operatorname{spin}(q+1) \cdot \operatorname{spin}\left(Q_{q}\right)=2 q .
$$

Thus, by recalling (3.54), the number $\sharp$ of scalar degrees of freedom on which the ADM mass depends along $\mathcal{O}_{\text {lightlike, } B P S}$ is (recall Eq. (3.64))

$$
\begin{aligned}
\sharp l i g h t, B P S & \equiv \operatorname{dim}_{\mathbb{R}} M_{5}-\operatorname{dim}_{\mathbb{R}} \mathcal{M}_{\text {lightlike, BPS }} \\
& =3 q+2-\left(\operatorname{spin}(q+1) \cdot \operatorname{spin}\left(Q_{q}\right)\right)=q+2 .
\end{aligned}
$$

By recalling Eq. (3.63), it is worth noting that $\mathcal{M}_{n B P S \text {,large }}$ and $\mathcal{M}_{\text {lightlike,BPS }}$ have the same real dimension, but they are completely different, as yielded by Eqs. (3.62) and (4.21).

- The "small" lightlike non-BPS charge orbit $\left(\operatorname{dim}_{\mathbb{R}}=3 q+2\right)$

$$
\mathcal{O}_{\text {lightlike, } n B P S}=\frac{G_{5}}{\left(S O(q, 1) \times \mathcal{A}_{q}\right) \rtimes \mathbb{R}^{\left(\operatorname{spin}(q+1), \operatorname{spin}\left(Q_{q}\right)\right)}},
$$

thus with

$$
\begin{aligned}
\mathcal{S}_{\text {max }, \text { lightlike }, n B P S} & =S O(q, 1) \times \mathcal{A}_{q} \\
\mathcal{T}_{\text {lightlike }, n B P S} & =\mathbb{R}^{\left(\operatorname{spin}(q+1), \operatorname{spin}\left(Q_{q}\right)\right)}=\mathcal{T}_{\text {lightlike }, B P S} .
\end{aligned}
$$

The related moduli space reads $\left(\operatorname{dim}_{\mathbb{R}}=3 q\right)$

$$
\begin{aligned}
\mathcal{M}_{\text {lightlike,nBPS }} & =\frac{S O(q, 1)}{S O(q)} \rtimes \mathbb{R}^{\left(\operatorname{spin}(q+1), \operatorname{spin}\left(Q_{q}\right)\right)} \\
& =M_{n J, 5, q} \rtimes \mathbb{R}^{\left(\operatorname{spin}(q+1), \operatorname{spin}\left(Q_{q}\right)\right)},
\end{aligned}
$$

where $M_{n J, 5, q}$ is the $q$-th element of the generic non-Jordan symmetric sequence (3.45). Thus, by recalling (3.54), the number $\sharp$ of scalar degrees of freedom on which the ADM mass depends along $\mathcal{O}_{\text {lightlike,nBPS }}$ is

$$
\begin{aligned}
\sharp_{\text {light }, n B P S} & \equiv \operatorname{dim}_{\mathbb{R}} M_{5}-\operatorname{dim}_{\mathbb{R}} \mathcal{M}_{\text {lightlike, }, \text { BPS }} \\
& =2 q+2-\left(\operatorname{spin}(q+1) \cdot \operatorname{spin}\left(Q_{q}\right)\right)=2 .
\end{aligned}
$$

- The "small" critical BPS charge orbit $\left(\operatorname{dim}_{\mathbb{R}}=2 q+1\right)$

$$
\mathcal{O}_{\text {critical }, B P S}=\frac{G_{5}}{\left(G_{6} \times \mathcal{A}_{q}\right) \rtimes \mathbb{R}^{\left(\operatorname{spin}(q+1), \operatorname{spin}\left(Q_{q}\right)\right)}},
$$

where

$$
G_{6}=S O(1, q+1)
$$

is the $U$-duality group of the corresponding $(1,0), d=6$ chiral supergravity theory. Thus:

$$
\begin{aligned}
& \mathcal{S}_{\text {max }, \text { critical }, B P S}=G_{6} \times \mathcal{A}_{q} ; \\
& \mathcal{T}_{\text {critical }, B P S}=\mathcal{T}_{\text {lightlike }, n B P S}=\mathcal{T}_{\text {lightlike }, B P S} .
\end{aligned}
$$


The related moduli space reads $\left(\operatorname{dim}_{\mathbb{R}}=3 q+1\right)$

$$
\begin{aligned}
\mathcal{M}_{\text {critical }, B P S} & =\frac{S O(q+1,1)}{S O(q+1)} \rtimes \mathbb{R}^{\left(\operatorname{spin}(q+1), \operatorname{spin}\left(Q_{q}\right)\right)} \\
& =M_{n J, 5, q+1} \rtimes \mathbb{R}^{\left(\operatorname{spin}(q+1), \operatorname{spin}\left(Q_{q}\right)\right)} .
\end{aligned}
$$

Thus, by recalling (3.54), the number $\sharp$ of scalar degrees of freedom on which the ADM mass depends along $\mathcal{O}_{\text {critical }, B P S}$ is

$$
\begin{aligned}
\sharp_{c r i t, B P S} & \equiv \operatorname{dim}_{\mathbb{R}} M_{5}-\operatorname{dim}_{\mathbb{R}} \mathcal{M}_{\text {critical }, B P S} \\
& =2 q+1-\left(\operatorname{spin}(q+1) \cdot \operatorname{spin}\left(Q_{q}\right)\right)=1 .
\end{aligned}
$$

The unique scalar degree of freedom on which the ADM mass depends can be interpreted as the Kaluza-Klein radius in the $d=5 \rightarrow d=4$ reduction. Furthermore, it is worth observing that:

$$
\mathcal{M}_{\text {critical }, B P S}=M_{(1,0), d=6, J_{3}^{\mathbb{A}}} \rtimes \mathbb{R}^{\left(\operatorname{spin}(q+1), \operatorname{spin}\left(Q_{q}\right)\right)},
$$

where $M_{(1,0), d=6, J_{3}^{\mathbb{A}}}$ is the manifold of tensor multiplets' scalars in the corresponding $(1,0)$, $d=6$ theory (see e.g. Sect. 5 of [20] for a recent treatment).

It should also be noticed that $\mathcal{O}_{n B P S \text {,large }}$ (given by Eq. $(3.59)$ ) and $\mathcal{O}_{\text {critical,BPS }}$ (given by

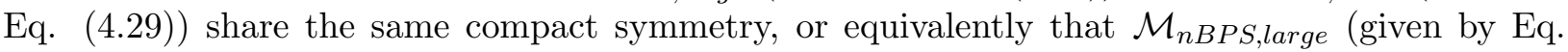
(3.62) and $\mathcal{M}_{\text {critical,BPS }}$ (given by Eq. 4.32) share the same stabilizer group (apart from an $\mathcal{A}_{q}$ commuting factor), but they do not coincide. This is due to the fact that $\widetilde{H}_{5}$ and $G_{6} \times \mathcal{A}_{q}$ share the same $m c s$, namely

$$
\widetilde{h}_{5} \equiv m c s\left(\widetilde{H}_{5}\right)=m c s\left(G_{6} \times \mathcal{A}_{q}\right)=S O(q+1) \times \mathcal{A}_{q} .
$$

In the case $\mathbb{A}=\mathbb{R}(q=1)$, the following further results holds (see also Tables 3 and 4 ):

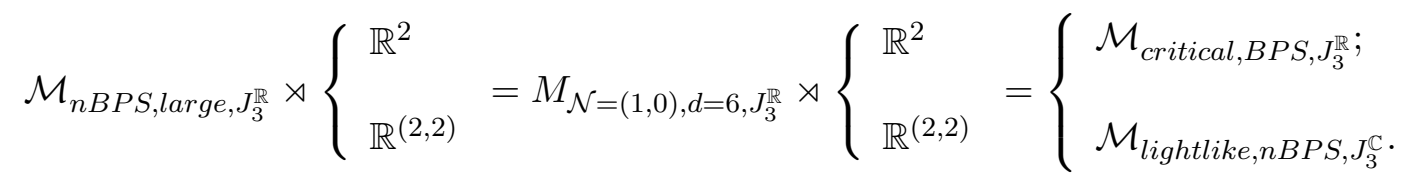

Notice that $J_{3}^{\mathbb{R}}$ is the unique case, among $J_{3}^{\mathbb{A}}$ in $d=5$, in which $\mathcal{M}_{n B P S \text {,large }}$ and $\mathcal{M}_{\text {critical,BPS }}$ not only share the same stabilizer, but they actually do coincide (up to $\rtimes \mathbb{R}^{2}$ ). Moreover, $\mathcal{M}_{n B P S, \text { large }, J_{3}^{\mathbb{R}}}$ also coincides with $\mathcal{M}_{\text {lightlike, } n B P S, J_{3}^{\mathbb{C}}}$ (up to $\rtimes \mathbb{R}^{(2,2)}$ ), because the respective charge orbits $\mathcal{O}_{n B P S, \text { large, } J_{3}^{\mathbb{R}}}$ and $\mathcal{O}_{\text {lightlike, } n B P S, J_{3}^{\mathbb{C}}}$ share the same semi-simple, namely non-translational, part of the stabilizer (apart from a commuting $\mathcal{A}_{2}=S O(2)$ factor), i.e. $S O(2,1)$.

The Jordan symmetric infinite sequence [28, 29, 30, 31, 32, 35, 36] given by Eq. (3.44) needs some extra care (also at the level of "large" charge orbits), because of the factorization of $G_{5}$. The "large" and "small" charge orbits for such a sequence will be treated in Sect. 6. This treatment refines and complete the ones given e.g. in [19, 23, 20]).

\section{1 $G_{5}$-invariant Defining Constraints}

As mentioned above, "small" charge orbits in all symmetric RSG are all characterized by the constraint (recall Eq. (3.48)):

$$
\widehat{\mathcal{I}}_{3}=\mathcal{I}_{3}=0,
$$




\begin{tabular}{|c|c|c|c|c|}
\hline $\begin{array}{c}J_{3}^{\mathbb{A}} \\
(+r e l . d a t a)\end{array}$ & $\begin{array}{c}\mathcal{O}_{\text {lightlike,BPS }} \\
r=2\end{array}$ & $\mathcal{M}_{\text {lightlike }, B P S}$ & $\begin{array}{c}\mathcal{O}_{\text {lightlike, } n B P S}, \\
r=2\end{array}$ & $\mathcal{M}_{\text {lightlike }, n B P S}$ \\
\hline $\begin{array}{l}\mathbb{A}=\mathbb{O}, q=8 \\
\operatorname{Spin}(9)=\mathbf{1 6} \\
\sharp l i g h t, B P S=10 \\
\sharp l i g h t, n B P S=2\end{array}$ & $\frac{E_{6(-26)}}{S O(9) \rtimes \mathbb{R}^{16}}$ & $\mathbb{R}^{16}$ & $\frac{E_{6(-26)}}{S O(8,1) \rtimes \mathbb{R}^{16}}$ & $\frac{S O(8,1)}{S O(8)} \rtimes \mathbb{R}^{16}$ \\
\hline $\begin{array}{l}\mathbb{A}=\mathbb{H}, q=4 \\
\mathcal{A}_{4}=S O(3) \\
Q_{4}=2 \\
\operatorname{Spin}(5)=\mathbf{4} \\
\operatorname{Spin}\left(Q_{4}\right)=\mathbf{2} \\
\sharp_{\text {light }, B P S}=6 \\
\sharp_{\text {light }, n B P S}=2\end{array}$ & $\frac{S U^{*}(6)}{(S O(5) \times S O(3)) \times \mathbb{R}^{(4,2)}}$ & $\mathbb{R}^{(4,2)}$ & $\frac{S U^{*}(6)}{(S O(4,1) \times S O(3)) \rtimes \mathbb{R}^{(4,2)}}$ & $\frac{S O(4,1)}{S O(4)} \rtimes \mathbb{R}^{(4,2)}$ \\
\hline $\begin{array}{l}\mathbb{A}=\mathbb{C}, q=2 \\
\mathcal{A}_{2}=S O(2) \\
Q_{2}=2, \\
\operatorname{Spin}(3)=\mathbf{2}, \\
\operatorname{Spin}\left(Q_{2}\right)=\mathbf{2} \\
\sharp_{l i g h t, B P S}=4 \\
\sharp_{l i g h t, n B P S}=2\end{array}$ & $\frac{S L(3, \mathbb{C})}{(S O(3) \times S O(2)) \rtimes \mathbb{R}^{(2,2)}}$ & $\mathbb{R}^{(2,2)}$ & $\frac{S L(3, \mathbb{C})}{(S O(2,1) \times S O(2)) \rtimes \mathbb{R}^{(2,2)}}$ & $\frac{S O(2,1)}{S O(2)} \rtimes \mathbb{R}^{(2,2)}$ \\
\hline $\begin{array}{l}\mathbb{A}=\mathbb{R}, q=1 \\
\operatorname{Spin}(2)=\mathbf{2} \\
\sharp \text { light }, B P S=3 \\
\sharp_{\text {light }, n B P S}=2\end{array}$ & $\frac{S L(3, \mathbb{R})}{S O(2) \rtimes \mathbb{R}^{2}}$ & $\mathbb{R}^{2}$ & $\frac{S L(3, \mathbb{R})}{S O(1,1) \rtimes \mathbb{R}^{2}}$ & $S O(1,1) \rtimes \mathbb{R}^{2}$ \\
\hline
\end{tabular}

Table 3: "Small" lightlike charge orbits $\mathcal{O}_{\text {lightlike,BPS }}$ and $\mathcal{O}_{\text {lightlike,nBPS }}$ (with associated moduli spaces) in symmetric "magic" RSG 


\begin{tabular}{|c|c|c|}
\hline $\begin{array}{c}J_{3}^{\mathbb{A}} \\
(+ \text { rel. data) }\end{array}$ & $\begin{array}{c}\mathcal{O}_{\text {critical }, B P S} \\
r=1\end{array}$ & $\mathcal{M}_{\text {critical }, B P S}$ \\
\hline $\begin{array}{c}\mathbb{A}=\mathbb{O}, q=8 \\
\operatorname{Spin}(9)=\mathbf{1 6} \\
\#_{c r i t}, B P S=1\end{array}$ & $\frac{E_{6(-26)}}{S O(9,1) \rtimes \mathbb{R}^{16}}$ & $\frac{S O(9,1)}{S O(9)} \rtimes \mathbb{R}^{16}$ \\
\hline $\begin{array}{l}\mathbb{A}=\mathbb{H}, q=4 \\
\mathcal{A}_{4}=S O(3), \\
Q_{4}=2, \\
\operatorname{Spin}(5)=\mathbf{4}, \\
\operatorname{Spin}\left(Q_{4}\right)=\mathbf{2} \\
\sharp_{c r i t}, B P S=1\end{array}$ & $\frac{S U^{*}(6)}{(S O(5,1) \times S O(3)) \rtimes \mathbb{R}^{(4,2)}}$ & $\frac{S O(5,1)}{S O(5)} \rtimes \mathbb{R}^{(4,2)}$ \\
\hline $\begin{array}{l}\mathbb{A}=\mathbb{C}, q=2 \\
\mathcal{A}_{2}=S O(2), \\
Q_{2}=2, \\
\operatorname{Spin}(3)=\mathbf{2}, \\
\operatorname{Spin}\left(Q_{2}\right)=\mathbf{2} \\
\sharp_{c r i t, B P S}=1\end{array}$ & $\frac{S L(3, \mathbb{C})}{(S O(3,1) \times S O(2)) \rtimes \mathbb{R}^{(2,2)}}$ & $\frac{S O(3,1)}{S O(3)} \rtimes \mathbb{R}^{(2,2)}$ \\
\hline $\begin{array}{l}\mathbb{A}=\mathbb{R}, q=1 \\
\operatorname{Spin}(2)=\mathbf{2} \\
\#_{c r i t, B P S}=1\end{array}$ & $\frac{S L(3, \mathbb{R})}{S O(2,1) \rtimes \mathbb{R}^{2}}$ & $\frac{S O(2,1)}{S O(2)} \rtimes \mathbb{R}^{2}$ \\
\hline
\end{tabular}

Table 4: "Small" critical charge orbit $\mathcal{O}_{\text {critical,BPS }}$ (with associated moduli space $\left.\mathcal{M}_{\text {critical,BPS }}\right)$ in symmetric "magic" RSG 
where $\widehat{\mathcal{I}}_{3}=\mathcal{I}_{3}$ is the unique cubic scalar invariant of the relevant electric representation $\mathbf{R}_{q}$ of the $d=5 U$-duality group $G_{5}$ (in which the electric charges $q_{i}$ 's sit). By recalling definitions (3.32) and (7.2), the "smallness" condition (4.37) can be recast as follows:

$$
\begin{aligned}
& \widehat{\mathcal{I}}_{3}=0 \Leftrightarrow Z^{3}-\left(\frac{3}{2}\right)^{2} Z Z_{x} Z^{x}-\left(\frac{3}{2}\right)^{\frac{3}{2}} T_{x y z} Z^{x} Z^{y} Z^{z}=0 ; \\
& \mathcal{I}_{3}=0 \Leftrightarrow d^{i j k} q_{i} q_{j} q_{k}=0,
\end{aligned}
$$

in the "dressed" and "bare" charges basis, respectively.

It is here worth noticing that Eq. (4.38) can be recast as a cubic algebraic equation:

$$
Z^{3}+\mathbf{p} Z-\mathbf{q}=0 ;\left\{\begin{array}{l}
\mathbf{p} \equiv-\left(\frac{3}{2}\right)^{2} Z_{x} Z^{x}<0 \\
\mathbf{q} \equiv\left(\frac{3}{2}\right)^{\frac{3}{2}} T_{x y z} Z^{x} Z^{y} Z^{z}
\end{array}\right.
$$

with polynomial discriminant

$$
D \equiv \frac{\mathbf{p}^{3}}{9}+\frac{\mathbf{q}^{2}}{4}=\frac{3^{3}}{2^{6}}\left[2\left(T_{x y z} Z^{x} Z^{y} Z^{z}\right)^{2}-\left(Z_{x} Z^{x}\right)^{3}\right] .
$$

Thus, for $D>0$ one gets one real and two complex conjugate (unacceptable) roots, whereas for $D<0$ all roots are real and unequal. In the particular case

$$
D=0 \Leftrightarrow 2\left(T_{x y z} Z^{x} Z^{y} Z^{z}\right)^{2}=\left(Z_{x} Z^{x}\right)^{3},
$$

all roots are real, and at least two equal.

Let us proceed further, by differentiating the functional $\widehat{\mathcal{I}}_{3}$ with respect to the "dressed" charges

$$
\mathcal{Z} \equiv\left\{Z, Z_{x}\right\}
$$

as well as function $\mathcal{I}_{3}$ with respect to the "bare" charges $\left\{q_{i}\right\}$. One respectively obtains:

$$
\begin{aligned}
& \frac{\partial \widehat{\mathcal{I}}_{3}}{\partial \mathcal{Z}}=\left\{\begin{array}{l}
\frac{\partial \widehat{\mathcal{I}}_{3}}{\partial Z}=\frac{1}{2} Z^{2}-\frac{3}{8} Z_{x} Z^{x} \\
\frac{\partial \widehat{\mathcal{I}}_{3}}{\partial Z_{x}}=-\frac{3}{4} Z Z^{x}-\frac{1}{2}\left(\frac{3}{2}\right)^{3 / 2} T_{y z}^{x} Z^{y} Z^{z}
\end{array}\right. \\
& \frac{\partial \mathcal{I}_{3}}{\partial q_{i}}=\frac{1}{2} d^{i j k} q_{j} q_{k},
\end{aligned}
$$

where it should be recalled once again that here we are considering symmetric real special manifolds $\frac{G_{5}}{H_{5}}$, where Eqs. 2.17) and 2.16 all hold true.

A further differentiation with respect to $\mathcal{Z}$ or $\left\{q_{i}\right\}$ respectively yields

$$
\begin{aligned}
& \frac{\partial^{2} \widehat{\mathcal{I}}_{3}}{(\partial \mathcal{Z})^{2}}=\left\{\begin{array}{l}
\frac{\partial^{2} \widehat{\mathcal{I}}_{3}}{(\partial Z)^{2}}=Z \\
\frac{\partial^{2} \widehat{\mathcal{I}}_{3}}{\partial Z \partial Z_{x}}=-\frac{3}{4} Z^{x} \\
\frac{\partial^{2} \widehat{\mathcal{I}}_{3}}{\partial Z_{x} \partial Z_{y}}=-\frac{3}{4} Z g^{x y}-\left(\frac{3}{2}\right)^{3 / 2} T_{z}^{x y} Z^{z}=\frac{\partial^{2} \widehat{\mathcal{I}}_{3}}{\left.\partial Z_{(x} \partial Z_{y}\right)}
\end{array}\right. \\
& \frac{\partial^{2} \mathcal{I}_{3}}{\partial q_{i} \partial q_{j}}=d^{i j k} q_{k}=\frac{\partial^{2} \mathcal{I}_{3}}{\left.\partial q_{(i} \partial q_{j}\right)} .
\end{aligned}
$$


By further differentiating, one then obtains:

$$
\begin{aligned}
& \frac{\partial^{3} \widehat{\mathcal{I}}_{3}}{(\partial \mathcal{Z})^{3}}=\left\{\begin{array}{l}
\frac{\partial^{3} \widehat{\mathcal{I}}_{3}}{(\partial Z)^{3}}=1 ; \\
\frac{\partial^{3} \widehat{\mathcal{I}}_{3}}{(\partial Z)^{2} \partial Z_{x}}=0 \\
\frac{\partial^{3} \widehat{\mathcal{I}}_{3}}{\partial Z \partial Z_{x} \partial Z_{y}}=-\frac{3}{4} g^{x y}=\frac{\partial^{3} \widehat{\mathcal{I}}_{3}}{\partial Z \partial Z_{(x} \partial Z_{y)}} ; \\
\frac{\partial^{3} \widehat{\mathcal{I}}_{3}}{\partial Z_{x} \partial Z_{y} \partial Z_{z}}=-\left(\frac{3}{2}\right)^{3 / 2} T^{x y z}=\frac{\partial^{3} \widehat{\mathcal{I}}_{3}}{\partial Z_{(x} \partial Z_{y} \partial Z_{z)}} ;
\end{array}\right. \\
& \frac{\partial^{3} \mathcal{I}_{3}}{\partial q_{i} \partial q_{j} \partial q_{k}}=d^{i j k}=\frac{\partial^{3} \mathcal{I}_{3}}{\partial q_{(i} \partial q_{j} \partial q_{k)}} \text {. }
\end{aligned}
$$

Starting from the fourth order of differentiation, all derivatives vanish. This is no surprise, because $\widehat{\mathcal{I}}_{3}$ is a functional polynomial homogeneous of degree three in "dressed" charges $\mathcal{Z}$, as well as (equivalently) $\mathcal{I}_{3}$ is a polynomial homogeneous of degree three in "bare" charges $q_{i}$ 's.

At this point, it is possible to classify the various "small" charge orbits through $G_{5}$-invariant conditions involving $\widehat{\mathcal{I}}_{3}$ and its non-vanishing functional derivatives with respect to $\mathcal{Z}$, or equivalently through $G_{5}$-invariant conditions involving $\mathcal{I}_{3}$ and its non-vanishing derivatives with respect to $q_{i}$ 's.

\subsection{1 "Small" Lightlike Orbits}

The "small" lightlike charge orbits are defined by the constraints (recall Eqs. 4.38 and 4.39)):

$$
\left\{\begin{array}{l}
\widehat{\mathcal{I}}_{3}=0 \Leftrightarrow Z^{3}-\left(\frac{3}{2}\right)^{2} Z Z_{x} Z^{x}-\left(\frac{3}{2}\right)^{\frac{3}{2}} T_{x y z} Z^{x} Z^{y} Z^{z}=0 ; \\
\frac{\partial \widehat{\mathcal{I}}_{3}}{\partial \mathcal{Z}} \neq 0 \Leftrightarrow\left\{\begin{array}{l}
Z^{2}-\frac{3}{4} Z_{x} Z^{x} \neq 0 \\
\text { and } / \text { or } \\
\left.Z Z^{x}+\sqrt{\frac{3}{2}} T_{y z}^{x} Z^{y} Z^{z} \neq 0 \text { (at least for some } x\right),
\end{array}\right.
\end{array}\right.
$$

or equivalently:

$$
\left\{\begin{array}{l}
\mathcal{I}_{3}=0 \Leftrightarrow d^{i j k} q_{i} q_{j} q_{k}=0 ; \\
\left.\frac{\partial \mathcal{I}_{3}}{\partial q_{i}} \neq 0 \Leftrightarrow d^{i j k} q_{j} q_{k} \neq 0 \text { (at least for some } i\right) ;
\end{array}\right.
$$

The sets of constraints (4.50) and (4.51) are both $G_{5}$-invariant, but their manifest invariance is different. Indeed, the "dressed" charge basis $\mathcal{Z}$ is covariant with respect to $H_{5}$, and thus the set of constraints (4.50) exhibits a manifest $H_{5}$-invariance. Instead, the "bare" charge basis $\left\{q_{i}\right\}$ is $G_{5}$-covariant, and thus the set of constraints 4.50 is manifestly $G_{5}$-invariant.

In the "dressed" charge basis, it is immediate to realize that two classes of "small" lightlike charge orbits exist, namely: 
- "small" lightlike charge orbit for which the constraints 4.50 are solved with $Z=0$ :

$$
\left\{\begin{array}{l}
\left.\widehat{\mathcal{I}}_{3}\right|_{Z=0}=0 \Leftrightarrow T_{x y z} Z^{x} Z^{y} Z^{z}=0 \\
\left.\frac{\partial \widehat{\mathcal{I}}_{3}}{\partial \mathcal{Z}}\right|_{Z=0} \neq 0 \Leftrightarrow\left\{\begin{array}{l}
Z_{x} Z^{x} \neq 0 \\
\text { and } / \text { or } \\
\left.T_{y z}^{x} Z^{y} Z^{z} \neq 0 \text { (at least for some } x\right) .
\end{array}\right.
\end{array}\right.
$$

Notice that the constraint $Z_{x} Z^{x} \neq 0$ is automatically satisfied, because: 1) $g_{x y}$ is assumed to be strictly positive definite, and 2) $Z_{x} \neq 0$ at least for some $x$ (otherwise, since $Z=0$, one would obtain the trivial limit in which all charges vanish).

- "small" lightlike charge orbit for which the constraints 4.50 ) are solved with $Z \neq 0$ (also recall Eqs. 4.40-4.42):

$$
\left\{\begin{array}{l}
\left.\widehat{\mathcal{I}}_{3}\right|_{Z \neq 0}=0 \Leftrightarrow Z^{3}-\left(\frac{3}{2}\right)^{2} Z Z_{x} Z^{x}-\left(\frac{3}{2}\right)^{\frac{3}{2}} T_{x y z} Z^{x} Z^{y} Z^{z}=0 \\
\left.\frac{\partial \widehat{\mathcal{I}}_{3}}{\partial \mathcal{Z}}\right|_{Z \neq 0} \neq 0 \Leftrightarrow\left\{\begin{array}{l}
Z^{2}-\frac{3}{4} Z_{x} Z^{x} \neq 0 \\
\text { and } / \text { or } \\
\left.Z Z^{x}+\sqrt{\frac{3}{2}} T_{y z}^{x} Z^{y} Z^{z} \neq 0 \text { (at least for some } x\right)
\end{array}\right.
\end{array}\right.
$$

\subsection{2 "Small" Critical Orbit}

The "small" critical charge orbit is defined by the constraints (recall Eqs. 4.38) and (4.39)):

$$
\left\{\begin{array}{l}
\widehat{\mathcal{I}}_{3}=0 \Leftrightarrow Z^{3}-\left(\frac{3}{2}\right)^{2} Z Z_{x} Z^{x}-\left(\frac{3}{2}\right)^{\frac{3}{2}} T_{x y z} Z^{x} Z^{y} Z^{z}=0 ; \\
\frac{\partial \widehat{\mathcal{I}}_{3}}{\partial \mathcal{Z}}=0 \Leftrightarrow\left\{\begin{array}{l}
Z^{2}-\frac{3}{4} Z_{x} Z^{x}=0 \\
Z Z^{x}+\sqrt{\frac{3}{2}} T_{y z}^{x} Z^{y} Z^{z}=0
\end{array}\right.
\end{array}\right.
$$

or equivalently:

$$
\left\{\begin{array}{l}
\mathcal{I}_{3}=0 \Leftrightarrow d^{i j k} q_{i} q_{j} q_{k}=0 ; \\
\frac{\partial \mathcal{I}_{3}}{\partial q_{i}}=0 \Leftrightarrow d^{i j k} q_{j} q_{k}=0 .
\end{array}\right.
$$

As noticed above for the sets of constraints 4.50 and (4.51), the sets of constraints (4.54) and (4.55) are both $G_{5}$-invariant: while (4.54) is manifestly invariant only under $H_{5}=m c s\left(G_{5}\right),(4.55)$ is actually manifestly $G_{5}$-invariant.

Once again, in the "dressed" charges basis it is immediate to realize that only one class of "small" critical charge orbits exists, namely: 
- "small" critical charge orbit for which the constraints (4.54) are solved with $Z \neq 0$ :

$$
\left\{\begin{array}{l}
\left.\widehat{\mathcal{I}}_{3}\right|_{Z \neq 0}=0 \Leftrightarrow Z^{3}-\left(\frac{3}{2}\right)^{2} Z Z_{x} Z^{x}-\left(\frac{3}{2}\right)^{\frac{3}{2}} T_{x y z} Z^{x} Z^{y} Z^{z}=0 ; \\
\left.\frac{\partial \widehat{\mathcal{I}}_{3}}{\partial \mathcal{Z}}\right|_{Z \neq 0}=0 \Leftrightarrow\left\{\begin{array}{l}
Z^{2}-\frac{3}{4} Z_{x} Z^{x}=0 \\
Z Z^{x}+\sqrt{\frac{3}{2}} T_{y z}^{x} Z^{y} Z^{z}=0 .
\end{array}\right.
\end{array}\right.
$$

Notice that, for the same reason the constraint $\left.\frac{\partial \widehat{\mathcal{I}}_{3}}{\partial Z}\right|_{Z=0} \neq 0$ is automatically satisfied for the "small" lightlike charge orbit whose a representative in the "dressed" charges basis is given by Eq. (4.52), a "small" critical charge orbit with representative having $Z=0$ cannot exist. Indeed, such an orbit should have $Z=0$ and $Z_{x} Z^{x}=0$. Due to the assumed strictly positive definiteness of $g_{x y}$, this would be possible only in the trivial limit of the theory in which all charges do vanish. This can be formally stated as follows:

$$
\left.\frac{\partial \widehat{\mathcal{I}}_{3}}{\partial \mathcal{Z}}\right|_{Z=0}=0 \Leftrightarrow \mathcal{Z}=0 .
$$

$5 \quad J_{3}^{\mathbb{H}}: \mathcal{N}=2$ versus $\mathcal{N}=6$

The rank-3 Euclidean Jordan algebra $J_{3}^{\mathbb{H}}(q=4)$ is related to two different theories, namely an $\mathcal{N}=2$ theory coupled to 14 Abelian vector multiplets and the $\mathcal{N}=6$ "pure" theory. These two theories share the same bosonic sector [15, 19, 59], but their fermionic sectors, exploiting the supersymmetric completion of the bosonic one, are different.

Thus, it also follows that the supersymmetry-preserving features of the "large" and "small" charge orbits of the relevant irrepr. 15 of $G_{5}=S U^{*}(6)$ are different. The $\mathcal{N}$-dependent supersymetry properties of the various orbits are given in Table 5 (notice they are consistent with the results of [53]). In the "large" (attractor) cases, these match the results of [20].

\section{$6 \mathcal{N}=2, d=5$ Jordan Symmetric Sequence}

The Jordan symmetric sequence of $\mathcal{N}=2, d=5$ supergravity coupled to $n_{V}=n+1$ vector multiplets reads $\left(\operatorname{dim}_{\mathbb{R}}=n+1, \operatorname{rank}=2, n \in \mathbb{N} \cup\{0\}\right)$

$$
M_{\mathcal{N}=2, d=5, \text { Jordan }, \text { symm }}=S O(1,1) \times \frac{S O(1, n)}{S O(n)} .
$$

This sequence is associated to the rank-3 Euclidean reducible Jordan algebra $\mathbb{R} \oplus \boldsymbol{\Gamma}_{1, n}$. In the following treatment, we will determine the "large" and "small" orbits of the irrepr. $(\mathbf{1}, \mathbf{1}+\mathbf{n})$ of the $U$-duality group $S O(1,1) \times S O(1, n)$.

For brevity's sake, we will do this only through an analysis in the "bare" charges' basis.

Without any loss in generality, one can choose to treat only $d=5$ extremal (electric) BHs. Indeed, due to the symmetricity of the reducible coset (6.1), the treatment of $d=5$ extremal (magnetic) black strings is essentially analogous.

Two disconnected geometric structures emerge in the treatment, namely: 


\begin{tabular}{|c|c|c|}
\hline$J_{3}^{\mathbb{H}}$ & $\mathcal{N}=2$ & $\mathcal{N}=6$ \\
\hline $\begin{array}{l}\frac{S U^{*}(6)}{U S p(6)} \\
\text { "large", } \mathcal{I}_{3} \neq 0\end{array}$ & $\frac{1}{2}-B P S$ & $\begin{array}{c}n B P S \\
Z_{A B, H}=0 \\
X_{H} \neq 0\end{array}$ \\
\hline $\begin{array}{l}\frac{S U^{*}(6)}{U S p(4,2)} \\
\text { "large", } \mathcal{I}_{3} \neq 0\end{array}$ & $n B P S, Z_{H} \neq 0$ & $\begin{array}{c}\frac{1}{6}-B P S \\
Z_{A B, H} \neq 0 \\
X_{H} \neq 0\end{array}$ \\
\hline $\begin{array}{c}\frac{S U^{*}(6)}{(S O(5) \times S O(3)) \rtimes \mathbb{R}^{(4,2)}} \\
\text { "small", } \mathcal{I}_{3}=0\end{array}$ & $\frac{1}{2}-B P S$ & $\frac{1}{6}-B P S$ \\
\hline $\begin{array}{c}\frac{S U^{*}(6)}{(S O(4,1) \times S O(3)) \rtimes \mathbb{R}^{(4,2)}} \\
\text { "small", } \mathcal{I}_{3}=0\end{array}$ & $n B P S$ & $\frac{1}{3}-B P S$ \\
\hline$\frac{S U^{*}(6)}{\frac{(S O(5,1) \times S O(3)) \rtimes \mathbb{R}^{(4,2)}}{\text { "small", } \partial \mathcal{I}_{3}=0}}$ & $\frac{1}{2}-B P S$ & $\frac{1}{2}-B P S$ \\
\hline
\end{tabular}

Table 5: $\mathcal{N}$-dependent supersymmetry-preserving features of "large" and "small" charge orbits of the irrepr. 15 of the $d=5 U$-duality group $S U^{*}(6)$, related to $J_{3}^{\mathbb{H}}$. This corresponds to two "twin" theories, sharing the same bosonic sector: an $\mathcal{N}=2$ Maxwell-Einstein theory and the $\mathcal{N}=6$ "pure" theory. The subscript " $H$ " stands for "(evaluated at the) horizon"

- Timelike two-sheet hyperboloid $T_{n}$, with the two disconnected sheets $T_{n}^{ \pm}$respectively related to $q_{0} \gtrless 0$ :

$$
\left.T_{n} \equiv \frac{S O(1, n)}{S O(n)}\right|_{q_{I}^{2}>0}=\underset{q_{0}>0}{T_{0}^{+}} \cup \underset{q_{0}<0}{T_{n}^{-}} ; T_{n}^{+} \cap T_{n}^{-}=\emptyset .
$$

- Forward/backward light-cone $\Lambda_{n}$ of $(n+1)$-dimensional Minkowski space with metric $\eta_{I J}$ defined by (6.5), with two (forward $\Lambda_{n}^{+}$and backward $\Lambda_{n}^{-}$) cone branches, respectively related to $q_{0} \gtrless 0$ :

$$
\Lambda_{n} \equiv \frac{S O(1, n)}{S O(n-1) \rtimes \mathbb{R}^{n-1}}=\Lambda_{n}^{+} \cup \Lambda_{n}^{-} ; \Lambda_{n}^{+} \cap \Lambda_{n}^{-}=0,
$$

0 here denoting the origin of $\Lambda_{n}$ itself.

Due to such structures, as well as to the lower $(\mathcal{N}=2)$ supersymmetry, the case study of "large" and "small" charge orbits in $\mathcal{N}=2, d=5$ Jordan symmetric sequence exhibits some subtleties absent in the $\mathcal{N}=4, d=5$ theory analyzed in Sect. 7 .

In the "bare" charges' basis, the electric cubic invariant of the $(\mathbf{1}, \mathbf{1}+\mathbf{n})$ of $S O(1,1) \times S O(1, n)$ reads as follows $(I=0, i$, where $i=1, \ldots, n$, throughout; " 0 " pertains to the $d=5$ graviphoton field, which through the dimensional reduction $d=5 \rightarrow d=4$ becomes the Maxwell vector field of 
the axio-dilatonic vector multiplet):

$$
\mathcal{I}_{3, e l} \equiv q_{H} q_{I} q_{J} \eta^{I J} \equiv q_{H} q_{I}^{2}=q_{H}\left(q_{0}^{2}-\sum_{i=1}^{n} q_{i}^{2}\right)
$$

where $q_{H}$ is the electric charge of the dilatonic vector multiplet: it is an $S O(1, n)$-singlet, with $S O(1,1)$-weight +2 . On the other hand, the $S O(1, n)$-vector $q_{I}$ has $S O(1,1)$-weight -1 , such that $\mathcal{I}_{3, e l}$ defined by $(6.4)$ is $S O(1,1) \times S O(1, n)$-invariant. Notice that the action of the $U$-duality group does not mix $q_{H}$ and $q_{I}$, and this originates more charge orbits with respect to the irreducible cases. Moreover, $\eta_{I J}=\eta^{I J}$ is the Lorentzian metric of $S O(1, n)$ :

$$
\eta_{I J}=\eta^{I J} \equiv \operatorname{diag}(+1, \overbrace{-1, \ldots,-1}^{n}) .
$$

In $\mathcal{N}=2, d=5$ Jordan symmetric sequence, as well as in $\mathcal{N}=4, d=5$ theory, the reducibility of the associated rank-3 Jordan algebra gives rise to many subtleties and differences with respect to the theories associated to irreducible Euclidean rank-3 Jordan algebras. In the $\mathcal{N}=2$ case under consideration, the major difference consists in a higher number of "large" and "small" orbits with respect to the "magic" supergravities.

\section{1 "Large" Orbits}

- BPS (3-charge) orbit, defined as follows:

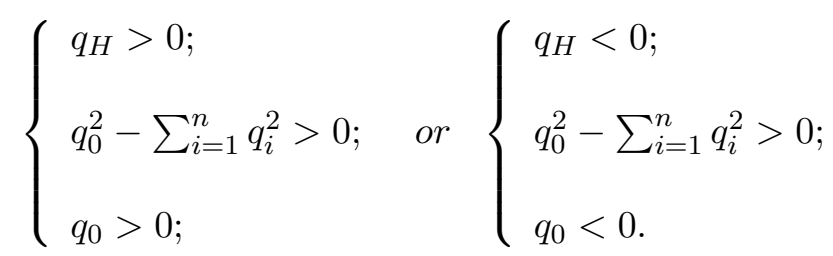

By recalling definition 6.2 , the orbit reads $(n \geqslant 0)$ :

$$
\mathcal{O}_{B P S, \text { large }}=\left[S O(1,1)^{+} \times T_{n}^{+}\right] \cup\left[S O(1,1)^{-} \times T_{n}^{-}\right],
$$

with no related moduli space. In particular, for $n=0$, namely in the so-called $\mathcal{N}=2, d=5$ $S O(1,1)$ model $\left(d=5\right.$ uplift of the $d=4 s t^{2}$ model $)$, in which only the dilatonic vector multiplet is coupled to the gravity multiplet, this orbit is actually 2-charge, and it is given by

$$
\mathcal{O}_{B P S, \text { large }, S O(1,1)}=\left\{\left(q_{H}, q_{0}\right)=(+,+),(-,-)\right\} .
$$

On the other hand, for $n=1$, i.e. in the so-called $\mathcal{N}=2, d=5[S O(1,1)]^{2}$ model $(d=5$ uplift of stu model), the cubic invariant (6.4) can be rewritten as follows:

$$
\begin{aligned}
& \mathcal{I}_{3, e l} \equiv q_{H} q_{I} q_{J} \eta^{I J} \equiv q_{H}\left(q_{0}^{2}-q_{1}^{2}\right)=q_{H} q_{+} q_{-} ; \\
& q_{ \pm} \equiv q_{0} \pm q_{1},
\end{aligned}
$$

and thus the hyperboloid (6.2) and light-cone (6.3) structures gets respectively factorized as follows ("+", "-" and "0" respectively denote strictly positive, strictly negative and vanishing 
values):

$$
\begin{aligned}
& T_{1}=\left.S O(1,1)\right|_{q_{+} q_{-}>0}=\underset{q_{0}>0}{T_{1}^{+}} \cup \underset{q_{0}<0}{\cup} T_{-}^{-} ; T_{1}^{+} \cap T_{1}^{-}=\emptyset \\
& \left\{\begin{array}{l}
T_{1}^{+}=\left\{\left(q_{+}, q_{-}\right)=(+,+)\right\} \\
T_{1}^{-}=\left\{\left(q_{+}, q_{-}\right)=(-,-)\right\}
\end{array}\right. \\
& \Lambda_{1}=S O(1,1)=\Lambda_{1}^{+} \cup \Lambda_{1}^{-} ; \Lambda_{1}^{+} \cap \Lambda_{1}^{-}=0 \\
& \left\{\begin{array}{l}
\Lambda_{1}^{+}=\left\{\left(q_{+}, q_{-}\right)=(+, 0),(0,+)\right\} \\
\Lambda_{1}^{-}=\left\{\left(q_{+}, q_{-}\right)=(-, 0),(0,-)\right\}
\end{array}\right.
\end{aligned}
$$

For $n=1$, orbit (6.7) reads

$$
\mathcal{O}_{B P S, 3-\operatorname{charge},[S O(1,1)]^{2}}=\left\{\left(q_{H}, q_{+}, q_{-}\right)=(+,+,+),(-,-,-)\right\} .
$$

This is invariant under triality permutation symmetry of $q_{H}, q_{+}$and $q_{-}$, and it is consistent with the analysis of [34].

- non-BPS (3-charge) orbit, with $Z \neq 0$ at the horizon, defined as follows:

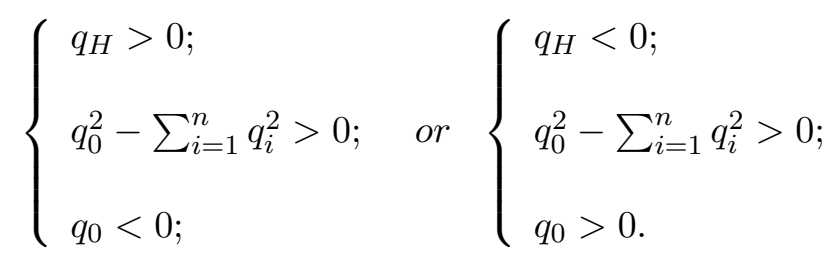

By recalling definition $(6.2)$, the orbit reads $(n \geqslant 0)$ :

$$
\mathcal{O}_{n B P S, \text { large }, I}=\left[S O(1,1)^{+} \times T_{n}^{-}\right] \cup\left[S O(1,1)^{-} \times T_{n}^{+}\right],
$$

with no related moduli space. In particular, for $n=0$, this orbit is actually 2-charge, and it is given by

$$
\mathcal{O}_{n B P S, l a r g e, S O(1,1)}=\left\{\left(q_{H}, q_{0}\right)=(+,-),(-,+)\right\} .
$$

On the other hand, for $n=1$, orbit 6.14 reads

$$
\mathcal{O}_{n B P S, l a r g e, I,[S O(1,1)]^{2}}=\left\{\left(q_{H}, q_{+}, q_{-}\right)=(+,-,-),(-,+,+)\right\} .
$$

The supersymmetry properties of $\mathcal{O}_{B P S \text {,large }}$ and $\mathcal{O}_{n B P S, \text { large,I }}$ can be understood by noticing that the flip of the sign of $q_{H}$ amounts, in the dressed charges' basis, to the exchange $Z \longleftrightarrow \partial_{s} Z$, where $s$ is the real dilaton scalar field, parametrizing $S O(1,1)$ of 6.1 .

It is worth pointing out both the $\mathcal{N}=2$ orbits $\mathcal{O}_{B P S \text {,large }}$ and $\mathcal{O}_{n B P S, \text { large,I }}$ (respectively given by $(6.7)$ and $(6.14)$ ) uplift to the same $\mathcal{N}=4$ orbit $\mathcal{O}_{\frac{1}{4}-B P S, \text { large, } \mathcal{N}=4, d=5}$ given by Eq. 7.4p. As mentioned, this is due to the fact that in $\mathcal{N}=4, d=5 q_{H}>0 \longleftrightarrow q_{H}<0$ amounts to exchanging the two gravitinos in the gravity multiplet, i.e. the two (opposite) skew-eigenvalues of the skew-traceless central charge matrix $\mathrm{Z}_{A B}(A, B=1, \ldots, 4)$. 
- Another non-BPS (3-charge) orbit, with $Z \neq 0$ at the horizon, is defined as follows [19]:

$$
\left\{\begin{array}{l}
q_{H} \gtrless 0 ; \\
q_{0}^{2}-\sum_{i=1}^{n} q_{i}^{2}<0 .
\end{array}\right.
$$

Thus, the resulting orbit reads (existing only for $n \geqslant 1$ )

$$
\mathcal{O}_{n B P S, \text { large }, I I}=S O(1,1) \times \frac{S O(1, n)}{S O(1, n-1)},
$$

with related moduli space (recall (3.44) and (3.45)):

$$
\begin{aligned}
\mathcal{M}_{n B P S, \text { large }, I I} & =\frac{S O(1, n-1)}{S O(n-1)} \\
& =\frac{M_{J, 5, n-1}}{S O(1,1)}=M_{n J, 5, n-1}=\left.M_{(1,0), d=6}\right|_{n-1},
\end{aligned}
$$

where $M_{n J, 5, n-1}$ denotes the $\mathcal{N}=2, d=5$ non-Jordan symmetric sequence with $n-1$ vector multiplets [41], and $\left.M_{(1,0), d=6}\right|_{n-1}$ is the scalar manifold of $(1,0), d=6$ supergravity with $n_{T}=n-1$ tensor multiplets. Thus, by recalling (6.1), the number $\sharp$ of "non-flat" scalar degrees of freedom along $\mathcal{O}_{n B P S, \text { large,II }}$ is independent on $n>1$ :

$$
\sharp_{n B P S, \text { large }, I I} \equiv \operatorname{dim}_{\mathbb{R}} M_{\mathcal{N}=2, d=5, \text { Jordan }, \text { symm }}-\operatorname{dim}_{\mathbb{R}} \mathcal{M}_{n B P S, \text { large }, I I}=2 .
$$

For $n=1$, orbit $(6.18)$ reads

$$
\mathcal{O}_{n B P S, \text { large }, I I,[S O(1,1)]^{2}}=\left\{\left(q_{H}, q_{+}, q_{-}\right)=(+,+,-),(+,-,+),(-,+,-),(-,-,+)\right\},
$$

with no corresponding moduli space. 6.21) is equivalent to 6.16 through triality permutation symmetry of $q_{H}, q_{+}$and $q_{-}$. Thus, consistent with the analysis of [34], the non-BPS "large" orbit of $[S O(1,1)]^{2}$ model is given, up to permutations of the triplet $\left(q_{H}, q_{+}, q_{-}\right)$, by

$$
\mathcal{O}_{n B P S, 3 \text {-charge },[S O(1,1)]^{2}}=\left\{\left(q_{H}, q_{+}, q_{-}\right)=(+,+,-),(+,-,-)\right\} .
$$

\section{2 "Small" Orbits}

Let us now consider the "small" orbits, and compute the criticality and double-criticality conditions on $\mathcal{I}_{3, e l}$ defined by (6.4):

$$
\begin{gathered}
\frac{\partial \mathcal{I}_{3, e l}}{\partial Q}=\left\{\begin{array}{l}
\frac{\partial \mathcal{I}_{3, e l}}{\partial q_{H}}=q_{I}^{2} \\
\frac{\partial \mathcal{I}_{3, e l}}{\partial q_{I}}=2 q_{H} q_{J} \eta^{I J}
\end{array}\right. \\
\frac{\partial^{2} \mathcal{I}_{3, e l}}{\partial Q^{2}}=\left\{\begin{array}{l}
\frac{\partial \mathcal{I}_{3, e l}}{\left(\partial q_{H}\right)^{2}}=0 ; \\
\frac{\partial \mathcal{I}_{3, e l}}{\partial q_{H} \partial q_{I}}=\frac{\partial \mathcal{I}_{3, e l}}{\partial q_{I} \partial q_{H}}=2 q_{J} \eta^{I J} \\
\frac{\partial \mathcal{I}_{3, e l}}{\partial q_{I} \partial q_{J}}=2 q_{H},
\end{array}\right.
\end{gathered}
$$


where

$$
Q \equiv\left(q_{H}, q_{I}\right)
$$

is shorthand for the vector of electric charges. As expected from the fact that $\mathcal{I}_{3, e l}$ is homogeneous of degree three, (6.24) implies that the unique doubly-critical orbit is the trivial one with all charges vanishing, because

$$
\frac{\partial^{2} \mathcal{I}_{3, e l}}{\partial Q^{2}}=0 \Leftrightarrow Q=0 .
$$

The "small" orbits of the $(\mathbf{1}, \mathbf{1}+\mathbf{n})$ of the $U$-duality group $S O(1,1) \times S O(1, n)$ list as follows:

1. BPS lightlike $\left(\mathcal{I}_{3, e l}=0, \frac{\partial \mathcal{I}_{3, e l}}{\partial Q} \neq 0: 2\right.$-charge) orbit with vanishing $q_{H}$ and timelike $q_{I}$ :

$$
\left\{\begin{array}{l}
q_{H}=0 ; \\
q_{0}^{2}-\sum_{i=1}^{n} q_{i}^{2}>0 .
\end{array}\right.
$$

By recalling definition $(6.2)$, the orbit reads $(n \geqslant 0)$ :

$$
\mathcal{O}_{B P S, \text { small }, I}=S O(1,1) \times T_{n},
$$

with no corresponding moduli space. In particular, for $n=0$ this orbit is actually 1-charge, and it is given by

$$
\mathcal{O}_{B P S, \text { small }, I, S O(1,1)}=\left\{\left(q_{H}, q_{0}\right)=(0,+),(0,-)\right\} .
$$

On the other hand, for $n=1$, the orbit 6.28 reads

$$
\mathcal{O}_{B P S, \text { small }, I,[S O(1,1)]^{2}}=\left\{\left(q_{H}, q_{+}, q_{-}\right)=(0,+,+),(0,-,-)\right\},
$$

with no corresponding moduli space, and thus

$$
\sharp_{B P S, \text { small }, I,[S O(1,1)]^{2}}=2 .
$$

2. Non-BPS lightlike $\left(\mathcal{I}_{3, e l}=0, \frac{\partial \mathcal{I}_{3, e l}}{\partial Q} \neq 0\right.$ : 2-charge) orbit with vanishing $q_{H}$ and spacelike $q_{I}$ :

$$
\left\{\begin{array}{l}
q_{H}=0 ; \\
q_{0}^{2}-\sum_{i=1}^{n} q_{i}^{2}<0 .
\end{array}\right.
$$

It reads (existing only for $n \geqslant 1$ )

$$
\mathcal{O}_{n B P S, \text { small }, I}=S O(1,1) \times \frac{S O(1, n)}{S O(1, n-1)},
$$

with corresponding moduli space (recall Eq. 6.19)

$$
\mathcal{M}_{n B P S, \text { small }, I}=\mathcal{M}_{n B P S, \text { large }, I I} .
$$

Thus, by recalling (6.1), the number $\sharp$ of "non-flat" scalar degrees of freedom along $\mathcal{O}_{n B P S, \text { small, } I}$ is independent on $n \geqslant 1$ :

$$
\sharp_{n B P S, \text { small }, I} \equiv \operatorname{dim}_{\mathbb{R}} M_{\mathcal{N}=2, d=5, \text { Jordan,symm }}-\operatorname{dim}_{\mathbb{R}} \mathcal{M}_{n B P S, \text { small }, I}=2 .
$$

For $n=1$, orbit 6.33 reads

$$
\mathcal{O}_{n B P S, \text { small }, I,[S O(1,1)]^{2}}=\left\{\left(q_{H}, q_{+}, q_{-}\right)=(0,+,-),(0,-,+)\right\},
$$

with no corresponding moduli space. 
3. BPS critical $\left(\mathcal{I}_{3, e l}=0, \frac{\partial \mathcal{I}_{3, e l}}{\partial Q}=0\right.$ : 1-charge) orbit with vanishing $q_{H}$ and lightlike $q_{I}$ :

$$
\left\{\begin{array}{l}
q_{H}=0 ; \\
q_{0}^{2}-\sum_{i=1}^{n} q_{i}^{2}=0 .
\end{array}\right.
$$

By recalling definition (6.3), the orbit reads (existing only for $n \geqslant 1$ )

$$
\mathcal{O}_{B P S, \text { small }, I I}=\Lambda_{n}
$$

and the corresponding moduli space is $(n \geqslant 1)$

$$
\mathcal{M}_{B P S, \text { small }, I I}=S O(1,1) \times \mathbb{R}^{n-1} .
$$

Thus, by recalling (6.1), the number $\sharp$ of "non-flat" scalar degrees of freedom along $\mathcal{O}_{B P S, \text { small,II }}$ is independent on $n \geqslant 1$ :

$$
\sharp_{B P S, \text { small }, I I} \equiv \operatorname{dim}_{\mathbb{R}} M_{\mathcal{N}=2, d=5, \text { Jordan,symm }}-\operatorname{dim}_{\mathbb{R}} \mathcal{M}_{B P S, \text { small }, I I}=1 .
$$

Analogously to what holds for symmetric "magic" RSG (noted below Eq. 4.33)), the unique scalar degree of freedom on which the ADM mass depends can be interpreted as the KaluzaKlein radius in the $d=5 \rightarrow d=4$ reduction. For $n=1$, orbit (6.38) reads

$$
\mathcal{O}_{B P S, \text { small }, I I,[S O(1,1)]^{2}}=\left\{\left(q_{H}, q_{+}, q_{-}\right)=(0,0,+),(0,+, 0),(0,0,-),(0,-, 0)\right\} .
$$

4. BPS lightlike $\left(\mathcal{I}_{3, e l}=0, \frac{\partial \mathcal{I}_{3, e l}}{\partial Q} \neq 0\right.$ : 2-charge $)$ orbit, defined as follows:

$$
\left\{\begin{array}{l}
q_{H}>0 ; \\
q_{0}^{2}-\sum_{i=1}^{n} q_{i}^{2}=0 ; \quad \text { or } \quad\left\{\begin{array}{l}
q_{H}<0 ; \\
q_{0}^{2}-0 ;
\end{array}\right. \\
q_{0}<0 . \sum_{i=1}^{n} q_{i}^{2}=0 ;
\end{array}\right.
$$

By recalling definition $(6.3)$, the orbit reads $(n \geqslant 2)$

$$
\mathcal{O}_{B P S, \text { small }, I I I}=\left[S O(1,1)^{+} \times \Lambda_{n}^{+}\right] \cup\left[S O(1,1)^{-} \times \Lambda_{n}^{-}\right],
$$

and the corresponding moduli space is purely translational $(n \geqslant 2)$ :

$$
\mathcal{M}_{B P S, \text { small,III }}=\mathbb{R}^{n-1}=\mathcal{M}_{B P S, \text { small,II} .}
$$

Thus, by recalling (6.1), the number $\sharp$ of "non-flat" scalar degrees of freedom along $\mathcal{O}_{B P S, \text { small,III }}$ is independent on $n \geqslant 2$ :

$$
\sharp_{B P S, \text { small }, I I I} \equiv \operatorname{dim}_{\mathbb{R}} M_{\mathcal{N}=2, d=5, \text { Jordan, symm }}-\operatorname{dim}_{\mathbb{R}} \mathcal{M}_{B P S, \text { small,III }}=2 .
$$

This orbit exists also for $n=1$, and it reads

$$
\mathcal{O}_{B P S, \text { small }, I I I,[S O(1,1)]^{2}}=\left\{\left(q_{H}, q_{+}, q_{-}\right)=(+, 0,+),(+,+, 0),(-, 0,-),(-,-, 0)\right\},
$$

with no corresponding moduli space. (6.46) is equivalent to (6.30) through triality permutation symmetry of $q_{H}, q_{+}$and $q_{-}$. Thus, the BPS 2-charge orbit of $[S O(1,1)]^{2}$ model is given, up to permutations of the triplet $\left(q_{H}, q_{+}, q_{-}\right)$, by

$$
\mathcal{O}_{B P S, 2 \text {-charge, }[S O(1,1)]^{2}}=\left\{\left(q_{H}, q_{+}, q_{-}\right)=(+,+, 0),(-,-, 0)\right\} .
$$


5. Non-BPS lightlike $\left(\mathcal{I}_{3, e l}=0, \frac{\partial \mathcal{I}_{3, e l}}{\partial Q} \neq 0\right.$ : 2-charge) orbit, defined as follows:

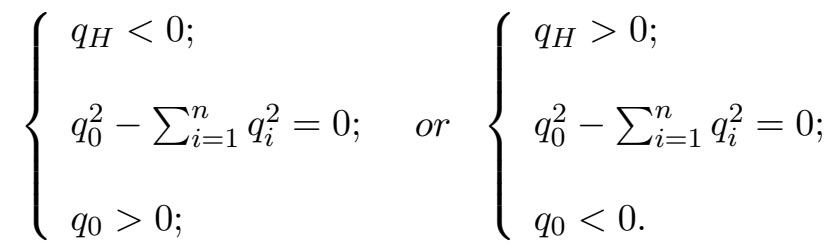

By recalling definition $(6.3)$, the orbit reads $(n \geqslant 2)$

$$
\mathcal{O}_{n B P S, \text { small }, I I}=\left[S O(1,1)^{+} \times \Lambda_{n}^{-}\right] \cup\left[S O(1,1)^{-} \times \Lambda_{n}^{+}\right],
$$

with corresponding moduli space $(n \geqslant 2)$

$$
\mathcal{M}_{n B P S, \text { small }, I I}=\mathbb{R}^{n-1}=\mathcal{M}_{B P S, \text { small }, I I}=\mathcal{M}_{B P S, \text { small }, I I I}
$$

Thus, by recalling (6.1), the number $\sharp$ of "non-flat" scalar degrees of freedom along $\mathcal{O}_{n B P S, s m a l l, I I}$ is independent on $n \geqslant 2$ :

$$
\sharp_{n B P S, \text { small }, I I} \equiv \operatorname{dim}_{\mathbb{R}} M_{\mathcal{N}=2, d=5, \text { Jordan,symm }}-\operatorname{dim}_{\mathbb{R}} \mathcal{M}_{n B P S, \text { small }, I I}=2 .
$$

This orbit exists also for $n=1$, and it reads

$$
\mathcal{O}_{n B P S, \text { small }, I I,[S O(1,1)]^{2}}=\left\{\left(q_{H}, q_{+}, q_{-}\right)=(+, 0,-),(+,-, 0),(-, 0,+),(-,+, 0)\right\},
$$

with no corresponding moduli space. 6.52$)$ is equivalent to $(6.36)$ through triality permutation symmetry of $q_{H}, q_{+}$and $q_{-}$. Thus, the non-BPS 2-charge orbit of $[S O(1,1)]^{2}$ model is given, up to permutations of the triplet $\left(q_{H}, q_{+}, q_{-}\right)$, by

$$
\mathcal{O}_{n B P S, 2 \text {-charge, }[S O(1,1)]^{2}}=\left\{\left(q_{H}, q_{+}, q_{-}\right)=(+,-, 0)\right\} .
$$

6. BPS critical $\left(\mathcal{I}_{3, e l}=0, \frac{\partial \mathcal{I}_{3, e l}}{\partial Q}=0: 1\right.$-charge $)$ orbit with vanishing $q_{I}$ and non-vanishing $q_{H}$ :

$$
\left\{\begin{array}{l}
q_{H} \in \mathbb{R}_{0} \\
q_{I}=0 .
\end{array}\right.
$$

It exists for every $n \geqslant 0$, and it reads

$$
\mathcal{O}_{B P S, \text { small }, I V}=S O(1,1),
$$

with moduli space $(n \geqslant 1$; recall 3.45$)$

$$
\mathcal{M}_{B P S, s m a l l, I V}=\frac{S O(1, n)}{S O(n)}=M_{n J, 5, n} .
$$

Thus, by recalling (6.1), the number $\sharp$ of "non-flat" scalar degrees of freedom along $\mathcal{O}_{B P S, \text { small,IV }}$ is independent on $n \geqslant 1$ :

$$
\sharp_{B P S, \text { small }, I V} \equiv \operatorname{dim}_{\mathbb{R}} M_{\mathcal{N}=2, d=5, \text { Jordan }, \text { symm }}-\mathcal{M}_{B P S, \text { small }, I V}=1 \text {. }
$$


Analogously to what holds for symmetric "magic" RSG (noted below Eq. 4.33)), the unique scalar degree of freedom on which the ADM mass depends can be interpreted as the KaluzaKlein radius in the $d=5 \rightarrow d=4$ reduction. Furthermore, as in the corresponding $\mathcal{N}=4$, $d=5$ "small" orbit (given by Eq. (7.34)), the sign of $q_{H}$ does not matter here. Orbit (6.55) is originated by the $d=6 \rightarrow d=5$ reduction of $(1,0)$ theory with all charges switched off. Indeed, $q_{H}$ is the electric charge of the Kaluza-Klein vector in the reduction $d=6 \rightarrow d=5$.

In particular, for $n=0$, this orbit reads

$$
\mathcal{O}_{B P S, \text { small }, I V, S O(1,1)}=\left\{\left(q_{H}, q_{0}\right)=(+, 0),(-, 0)\right\},
$$

with no corresponding moduli space. On the other hand, for $n=1$ the orbit 6.55 reads

$$
\mathcal{O}_{B P S, s m a l l, I V,[S O(1,1)]^{2}}=\left\{\left(q_{H}, q_{+}, q_{-}\right)=(+, 0,0),(-, 0,0)\right\},
$$

which is equivalent to (6.41) through triality permutation symmetry of $q_{H}, q_{+}$and $q_{-}$. Thus, the BPS 1-charge orbit of $[S O(1,1)]^{2}$ model is given, up to permutations of the triplet $\left(q_{H}, q_{+}, q_{-}\right)$, by

$$
\mathcal{O}_{B P S, 1-\text { charge },[S O(1,1)]^{2}}=\left\{\left(q_{H}, q_{+}, q_{-}\right)=(+, 0,0),(-, 0,0)\right\} .
$$

Thus, the stratification structure of the $(\mathbf{1}, \mathbf{1}+\mathbf{n})$-repr. space of the $d=5 U$-duality group $S O(1,1) \times S O(1, n)$ can be given through the following two chains of relations, proceeding (left to right) from 1-charge orbits to 2-charge and then 3-charge orbits:

$$
\begin{aligned}
\mathcal{O}_{B P S, \text { small }, I I} \rightarrow\left\{\begin{aligned}
& \mathcal{O}_{B P S, \text { small }, I} \rightarrow\left\{\begin{array}{l}
\mathcal{O}_{B P S, \text { large }} \\
\mathcal{O}_{n B P S, \text { large }, I}
\end{array}\right. \\
& \mathcal{O}_{n B P S, \text { small }, I} \rightarrow \mathcal{O}_{n B P S, \text { large }, I I} \\
& \mathcal{O}_{B P S, \text { small }, I I I}
\end{aligned}\right. \\
\mathcal{O}_{B P S, \text { small }, I V} \rightarrow\left\{\begin{array}{l}
\mathcal{O}_{B P S, \text { large }} \\
\mathcal{O}_{n B P S, \text { large }, I I}
\end{array}\right. \\
\mathcal{O}_{n B P S, \text { small }, I I} \rightarrow\left\{\begin{array}{l}
\mathcal{O}_{n B P S, \text { large }, I} \\
\mathcal{O}_{n B P S, \text { large }, I I}
\end{array}\right. \\
\mathcal{O}_{B P S, \text { small }, I I I} \rightarrow\left\{\begin{array}{l}
\mathcal{O}_{B P S, \text { large }} \\
\mathcal{O}_{n B P S, \text { large }, I I}
\end{array}\right. \\
\mathcal{O}_{n B P S, \text { small }, I I} \rightarrow\left\{\begin{array}{l}
\mathcal{O}_{n B P S, \text { large }, I} \\
\mathcal{O}_{n B P S, \text { large }, I I} .
\end{array}\right.
\end{aligned}
$$

For the $S O(1,1)$ model $(n=0)$, such a stratification structure simplifies as follows:

$$
\left.S O(1,1): \begin{array}{c}
\text { 1-charge } \\
\mathcal{O}_{B P S, \text { small }, I} \\
\mathcal{O}_{B P S, \text { small }, I V}
\end{array}\right\} \rightarrow\left\{\begin{array}{c}
\mathcal{O}_{B P S \text {, large }} \\
\mathcal{O}_{n B P S, \text { large }} .
\end{array}\right.
$$


On the other hand, for the $[S O(1,1)]^{2}$ model $(n=1)$, stratification structure $6.61-6.62$ reads:

$$
[S O(1,1)]^{2}: \mathcal{O}_{B P S, 1-\text { charge }} \rightarrow\left\{\begin{array}{l}
\mathcal{O}_{B P S, 2 \text {-charge }} \rightarrow\left\{\begin{array}{l}
\mathcal{O}_{B P S, 3-\text { charge }} \\
\mathcal{O}_{n B P S, 3-\text { charge }}
\end{array}\right. \\
\mathcal{O}_{n B P S, 2 \text {-charge }} \rightarrow \mathcal{O}_{n B P S, 3 \text {-charge }}
\end{array}\right.
$$

Thus, summarizing, $\mathcal{N}=2, d=5$ Jordan symmetric sequence admits six "small" charge orbits describing the flux configurations supporting static, spherically symmetric, asymptotically flat "small" BHs: four $\frac{1}{2}$-BPS and two non-BPS. Furthermore, the "large" orbits are three, namely one $\frac{1}{2}$-BPS and two non-BPS (with $Z \neq 0$ at the horizon).

\section{$7 \mathcal{N}=4, d=5$ Supergravity}

The scalar manifold of $\mathcal{N}=4, d=5$ supergravity coupled to $n_{V}=n \in \mathbb{N} \cup\{0\}$ matter (vector) multiplets reads $\left(\operatorname{dim}_{\mathbb{R}}=1+5 n, \operatorname{rank}=1+\min (5, n)\right)$

$$
M_{\mathcal{N}=4, d=5}=S O(1,1) \times \frac{S O(5, n)}{S O(5) \times S O(n)} .
$$

This theory is associated to the rank-3 Euclidean reducible Jordan algebra $\mathbb{R} \oplus \boldsymbol{\Gamma}_{5, n}$. In the following treatment, we will determine the "large" and "small" orbits of the irrepr. $(\mathbf{1}, \mathbf{5}+\mathbf{n})$ of the $U$-duality group $S O(1,1) \times S O(5, n)$.

For brevity's sake, we will do this only through an analysis in the "bare" charges' basis.

Without any loss in generality, one can choose to treat only $d=5$ extremal (electric) BHs. Indeed, due to the symmetricity of the reducible coset (7.1), the treatment of $d=5$ extremal (magnetic) black strings is essentially analogous.

In the "bare" charges' basis, the electric cubic invariant of the $(\mathbf{1}, \mathbf{5}+\mathbf{n})$ of $S O(1,1) \times S O(5, n)$ reads as follows $(I=1, \ldots, 5+n$ throughout; the indices $1, \ldots, 5$, with positive signature, pertain to the five $\mathcal{N}=4, d=5$ graviphotons):

$$
\mathcal{I}_{3, e l} \equiv q_{H} q_{I} q_{J} \eta^{I J} \equiv q_{H} q_{I}^{2},
$$

where $q_{H}$ is the electric charge of the 3 -form field strength of the 2 -form $B_{\mu \nu}(\mu, \nu=0,1, \ldots, 4)$ in the gravity multiplet (see e.g. [60, 61]). $q_{H}$ is an $S O(5, n)$-singlet, with $S O(1,1)$-weight +2 . On the other hand, the $S O(5, n)$-vector $q_{I}$ has $S O(1,1)$-weight -1 , such that $\mathcal{I}_{3, e l}$ defined by $(7.2)$ is $S O(1,1) \times S O(5, n)$-invariant. Notice that the action of the $U$-duality group does not mix $q_{H}$ and $q_{I}$, and this originates more charge orbits with respect to the irreducible cases. Moreover, $\eta_{I J}=\eta^{I J}$ is the pseudo-Euclidean metric of $S O(5, n)$, with signature $(\overbrace{+, \ldots,+,}^{5}, \overbrace{-, \ldots,-}^{n})$.

\section{1 "Large" Orbits}

- $\frac{1}{4}$-BPS (3-charge) orbit, defined by a timelike $q_{I}$ vector, with $q_{H}$ of any sign:

$$
q_{H} \in \mathbb{R}_{0}, q_{I} q_{J} \eta^{I J}>0 .
$$


The resulting form of the orbit reads $[20](n \geqslant 0)$

$$
\mathcal{O}_{\frac{1}{4}-B P S, \text { large }}=S O(1,1) \times \frac{S O(5, n)}{S O(4, n)},
$$

with related moduli space:

$$
\begin{aligned}
\mathcal{M}_{\frac{1}{4}-B P S, \text { large }} & =\frac{S O(4, n)}{S O(4) \times S O(n)} \\
& =\frac{M_{(1,1), d=6}}{S O(1,1)},
\end{aligned}
$$

where $M_{(1,1), d=6}$ is the scalar manifold of non-chiral half-maximal supergravity in $d=6$ with $n$ matter (vector) multiplets. The exchange between $q_{H}>0$ and $q_{H}<0$ amounts to exchanging the two gravitinos in the gravity multiplet, i.e. the two (opposite) skew-eigenvalues of the skew-traceless central charge matrix $\stackrel{\circ}{\mathrm{Z}}_{A B}(A, B=1, \ldots, 4)$. Thus, the number $\sharp$ of "non-flat" scalar degrees of freedom along $\mathcal{O}_{\frac{1}{4}-B P S \text {,large }}$ is (for $n \geqslant 1$ )

$$
\sharp_{\frac{1}{4}-B P S, \text { large }} \equiv \operatorname{dim}_{\mathbb{R}} M_{\mathcal{N}=4, d=5}-\operatorname{dim}_{\mathbb{R}} \mathcal{M}_{\frac{1}{4}-B P S, \text { large }}=n+1 \text {. }
$$

In $\mathcal{N}>2$-extended supergravity theories, in general $\frac{1}{\mathcal{N}}$-BPS attractors have a related moduli space [23. It corresponds to the hypermultiplets' scalar manifold in the supersymmetry reduction $\mathcal{N}>2 \longrightarrow \mathcal{N}=2$ of the theory under consideration. In this case, it is amusing to observe that $\mathcal{M}_{\frac{1}{4}-B P S \text {,large }}$ given by 7.5 is the $c$-map of the vector multiplets' scalar manifold of the $\mathcal{N}=2, d=4$ Jordan symmetric sequence:

$$
\mathcal{M}_{\frac{1}{4}-B P S, \text { large }}=c\left(\frac{S U(1,1)}{U(1)} \times \frac{S O(2, n-2)}{S O(2) \times S O(n-2)}\right) .
$$

Thus, $\mathcal{M}_{\frac{1}{4}-B P S, \text { large }}$ admits an interpretation either as $\mathbf{1}$ ) scalar manifold of $\mathcal{N}=4, d=$ 3 Jordan symmetric sequence in $d=3$, or as 2) the hypermultiplets' scalar manifold of Jordan symmetric sequence in $d=4,5(\mathcal{N}=2)$ and $6((1,0))$. In particular, $\mathcal{M}_{\frac{1}{4}-B P S \text {,large }}$ parametrizes the $\mathcal{N}=2$ hyperscalar degrees of freedom in the supersymmetry/Jordan algebra reduction:

$$
d=5: \begin{gathered}
\mathcal{N}=4 \\
\mathbb{R} \oplus \boldsymbol{\Gamma}_{5, n}
\end{gathered} \longrightarrow \begin{gathered}
\mathcal{N}=2 \\
\mathbb{R} \oplus \boldsymbol{\Gamma}_{1, n-3}
\end{gathered} .
$$

The pure theory (i.e. $n=0$ ) limit of orbit $(7.4)$ is actually 2-charge (indeed, $S O(5)$ symmetry can be used to make only one component of the Euclidean vector $q_{I}$ non-vanishing), and it reads

$$
\mathcal{O}_{\frac{1}{4}-B P S, \text { large }, n=0}=S O(1,1) \times \frac{S O(5)}{S O(4)} \equiv S O(1,1) \times S^{4},
$$

with no corresponding moduli space, and thus trivially

$$
\sharp_{\frac{1}{4}}-B P S, \text { large }, n=0=1 \text {. }
$$

- non-BPS (3-charge) orbit with $\stackrel{\circ}{Z}_{A B}=0$ (at the horizon), defined by a spacelike $q_{I}$ vector, and $q_{H}$ of any sign:

$$
q_{H} \in \mathbb{R}_{0}, q_{I} q_{J} \eta^{I J}<0 .
$$


Notice that both signs of $q_{H}$ are allowed, due to the fact that the non-BPS $\AA_{A B}=0$ Attractor Eqs. are quadratic in $q_{H}$ (see e.g. [20]). The resulting orbit reads $(n \geqslant 1$, not existing in pure theory) [20]

$$
\mathcal{O}_{n B P S, \text { large }}=S O(1,1) \times \frac{S O(5, n)}{S O(5, n-1)},
$$

with related moduli space:

$$
\begin{aligned}
\mathcal{M}_{n B P S, \text { large }} & =\frac{S O(5, n-1)}{S O(5) \times S O(n-1)} \\
& =\left.M_{(2,0), d=6}\right|_{n-1},
\end{aligned}
$$

where $\left.M_{(2,0), d=6}\right|_{n-1}$ is the scalar manifold of $(2,0), d=6$ supergravity with $n_{T}=n-1$ tensor multiplets. Note that $\mathcal{N}=4, d=5$ and $(2,0), d=6$ supergravities share the same $\mathcal{R}$-symmetry $S O(5) \sim U S p(4)$. Thus, the number $\sharp$ of "non-flat" scalar degrees of freedom along $\mathcal{O}_{n B P S \text {,large }}$ is independent on $n \geqslant 2$ :

$$
\sharp_{n B P S, \text { large }} \equiv \operatorname{dim}_{\mathbb{R}} M_{\mathcal{N}=4, d=5}-\operatorname{dim}_{\mathbb{R}} \mathcal{M}_{n B P S \text {,large }}=6 .
$$

\section{2 "Small" Orbits}

The conditions on $\mathcal{I}_{3, e l}$ defined by $(7.2)$ are formally the same as the ones holding in $\mathcal{N}=2, d=5$ Jordan symmetric sequence, and given by Eqs. (6.23) and (6.24). Thus, analogously to the case of $\mathcal{N}=2, d=5$ Jordan symmetric sequence, and as expected from the fact that $\mathcal{I}_{3, e l}$ is homogeneous of degree three, (6.24) implies that the unique doubly-critical orbit is the trivial one with all charges vanishing (namely, 0-charge orbit; recall Eq. (6.26)).

The "small" orbits of the $(\mathbf{1}, \mathbf{5}+\mathbf{n})$ of the $U$-duality group $S O(1,1) \times S O(5, n)$ list as follows:

1. Lightlike $\left(\mathcal{I}_{3, e l}=0, \frac{\partial \mathcal{I}_{3, e l}}{\partial Q} \neq 0\right.$ : 2-charge) orbit with vanishing $q_{H}$ and timelike $q_{I}$ :

$$
\left\{\begin{array}{l}
q_{H}=0 \\
q_{I}^{2}>0
\end{array}\right.
$$

This orbit is $\frac{1}{2}$-BPS [14]. It reads $(n \geqslant 0)$

$$
\mathcal{O}_{\frac{1}{2}-B P S, \text { small }, I}=S O(1,1) \times \frac{S O(5, n)}{S O(4, n)},
$$

with corresponding moduli space (recall Eq. (7.5))

$$
\mathcal{M}_{\frac{1}{2}-B P S, \text { small,I}}=\mathcal{M}_{\frac{1}{4}-B P S, \text { large }} .
$$

Thus, the number $\sharp$ of "non-flat" scalar degrees of freedom along $\mathcal{O}_{\frac{1}{2}-B P S, \text { small,I}}$ is (for $n \geqslant 1$ ):

$$
\sharp_{\frac{1}{2}-B P S, \text { small }, I} \equiv \operatorname{dim}_{\mathbb{R}} M_{\mathcal{N}=4, d=5}-\operatorname{dim}_{\mathbb{R}} \mathcal{M}_{\frac{1}{2}-B P S, \text { small }, I}=n+1 .
$$

The pure theory (i.e. $n=0$ ) limit of orbit (7.16) is actually 1-charge, and it reads

$$
\mathcal{O}_{\frac{1}{2}-B P S, \text { small }, I, n=0}=S O(1,1) \times S^{4},
$$


with no related moduli space, and thus

$$
\sharp_{\frac{1}{2}-B P S, \text { small }, I, n=0}=1 \text {. }
$$

2. Lightlike $\left(\mathcal{I}_{3, e l}=0, \frac{\partial \mathcal{I}_{3, e l}}{\partial Q} \neq 0: 2\right.$-charge $)$ orbit with vanishing $q_{H}$ and spacelike $q_{I}$ :

$$
\left\{\begin{array}{l}
q_{H}=0 \\
q_{I}^{2}<0
\end{array}\right.
$$

This orbit is non-BPS. It reads ( $n \geqslant 1$, not existing in pure theory)

$$
\mathcal{O}_{n B P S, \text { small }}=S O(1,1) \times \frac{S O(5, n)}{S O(5, n-1)},
$$

with corresponding moduli space (recall Eq. (7.13))

$$
\mathcal{M}_{n B P S, \text { small }}=\mathcal{M}_{n B P S, \text { large }} .
$$

Thus, the number $\sharp$ of "non-flat" scalar degrees of freedom along $\mathcal{O}_{n B P S \text {,small }}$ is independent on $n \geqslant 1$ :

$$
\sharp_{n B P S, \text { small }} \equiv \operatorname{dim}_{\mathbb{R}} M_{\mathcal{N}=4, d=5}-\operatorname{dim}_{\mathbb{R}} \mathcal{M}_{n B P S, \text { small }}=6 .
$$

3. Critical $\left(\mathcal{I}_{3, e l}=0, \frac{\partial \mathcal{I}_{3, e l}}{\partial Q}=0\right.$ : 1-charge) orbit with vanishing $q_{H}$ and lightlike $q_{I}$ :

$$
\left\{\begin{array}{l}
q_{H}=0 \\
q_{I}^{2}=0
\end{array}\right.
$$

This orbit is $\frac{1}{2}$-BPS [14]. It reads $(n \geqslant 1$, not existing in pure theory)

$$
\mathcal{O}_{\frac{1}{2}-B P S, \text { small }, I I}=\frac{S O(5, n)}{S O(4, n-1) \rtimes \mathbb{R}^{4, n-1}},
$$

with corresponding moduli space (recall Eq. (7.17))

$$
\begin{aligned}
\mathcal{M}_{\frac{1}{2}-B P S, \text { small }, I I} & =S O(1,1) \times\left.\mathcal{M}_{\frac{1}{2}-B P S, \text { small }, I}\right|_{n \rightarrow n-1} \rtimes \mathbb{R}^{4, n-1} \\
& =S O(1,1) \times\left.\mathcal{M}_{\frac{1}{4}-B P S, \text { large }}\right|_{n \rightarrow n-1} \rtimes \mathbb{R}^{4, n-1} .
\end{aligned}
$$

Thus, the number $\sharp$ of "non-flat" scalar degrees of freedom along $\mathcal{O}_{\frac{1}{2}-B P S, \text { small,II}}$ is independent on $n \geqslant 1$ :

$$
\sharp_{\frac{1}{2}-B P S, \text { small }, I I} \equiv \operatorname{dim}_{\mathbb{R}} M_{\mathcal{N}=4, d=5}-\operatorname{dim}_{\mathbb{R}} \mathcal{M}_{\frac{1}{2}-B P S, \text { small,II }}=1 .
$$

Analogously to what holds for symmetric "magic" RSG (noted below Eq. (4.33)) and for $N=2, d=5$ Jordan symmetric sequence treated in Sect. 6, the unique scalar degree of freedom on which the ADM mass depends can be interpreted as the Kaluza-Klein radius in the $d=5 \rightarrow d=4$ reduction. 
4. Lightlike $\left(\mathcal{I}_{3, e l}=0, \frac{\partial \mathcal{I}_{3, e l}}{\partial Q} \neq 0\right.$ : 2-charge $)$ orbit with non-vanishing $q_{H}$ and lightlike $q_{I}$ :

$$
\left\{\begin{array}{l}
q_{H} \in \mathbb{R}_{0} ; \\
q_{I}^{2}=0 .
\end{array}\right.
$$

This orbit is $\frac{1}{4}$-BPS. It reads $(n \geqslant 1)$

$$
\mathcal{O}_{\frac{1}{4}-B P S, \text { small }}=S O(1,1) \times \frac{S O(5, n)}{S O(4, n-1) \rtimes \mathbb{R}^{4, n-1}},
$$

with corresponding moduli space (recall Eq. (7.27))

$$
\begin{aligned}
\mathcal{M}_{\frac{1}{4}-B P S, \text { small }} & =\left.\mathcal{M}_{\frac{1}{2}-B P S, \text { small }, I}\right|_{n \rightarrow n-1} \rtimes \mathbb{R}^{4, n-1} \\
& =\left.\mathcal{M}_{\frac{1}{4}-B P S \text {, large }}\right|_{n \rightarrow n-1} \rtimes \mathbb{R}^{4, n-1} .
\end{aligned}
$$

Thus, the number $\sharp$ of "non-flat" scalar degrees of freedom along $\mathcal{O}_{\frac{1}{2}-B P S, \text { small,II}}$ is independent on $n \geqslant 1$ :

$$
\sharp_{\frac{1}{4}-B P S, \text { small }} \equiv \operatorname{dim}_{\mathbb{R}} M_{\mathcal{N}=4, d=5}-\operatorname{dim}_{\mathbb{R}} \mathcal{M}_{\frac{1}{4}-B P S, \text { small }}=2 .
$$

5. Critical $\left(\mathcal{I}_{3, e l}=0, \frac{\partial \mathcal{I}_{3, e l}}{\partial Q}=0\right.$ : 1-charge) orbit with vanishing $q_{I}$ and non-vanishing $q_{H}$ :

$$
\left\{\begin{array}{l}
q_{H} \in \mathbb{R}_{0} \\
q_{I}=0 .
\end{array}\right.
$$

This orbit is $\frac{1}{2}$-BPS [14]. It reads (independent on $n \geqslant 0$ )

$$
\mathcal{O}_{\frac{1}{2}-B P S, \text { small,III }}=S O(1,1),
$$

with moduli space

$$
\mathcal{M}_{\frac{1}{2}-B P S, \text { small }, I I I}=\frac{S O(5, n)}{S O(5) \times S O(n)} .
$$

Thus, the number $\sharp$ of "non-flat" scalar degrees of freedom along $\mathcal{O}_{\frac{1}{2}-B P S, \text { small,III }}$ is independent on $n \geqslant 0$ :

$$
\sharp_{\frac{1}{2}-B P S, \text { small,III }} \equiv \operatorname{dim}_{\mathbb{R}} M_{\mathcal{N}=4, d=5}-\mathcal{M}_{\frac{1}{2}-B P S, \text { small }, I I I}=1 \text {. }
$$

Notice that $\mathcal{O}_{\frac{1}{2}-B P S, \text { small,III }}$ can also be seen as the " $n=0$ formal limit" of $\mathcal{O}_{\frac{1}{4}-B P S, \text { small }}$ given by Eq. 7.30). Indeed, the $n=0$ limit of 7.29) is given by 7.33 itself. Furthermore, analogously to what holds for symmetric "magic" RSG (noted below Eq. 4.33) and for $N=2, d=5$ Jordan symmetric sequence treated in Sect. 6, the unique scalar degree of freedom on which the ADM mass depends can be interpreted as the Kaluza-Klein radius in the $d=5 \rightarrow d=4$ reduction. Orbit (7.34) is originated by the $d=6 \rightarrow d=5$ reduction of $(2,0)$ theory with all charges switched off. Indeed, $q_{H}$ is the electric charge of the KaluzaKlein vector in the reduction $d=6 \rightarrow d=5$. Notice that in the pure theory (i.e. $n=0$ ) $\mathcal{M}_{\frac{1}{2}-\text { BPS,small,III }}$ vanishes, and thus:

$$
\sharp_{\frac{1}{2}-B P S, \text { small }, I I I, n=0}=1 .
$$


Thus, the stratification structure of the $(\mathbf{1}, \mathbf{5}+\mathbf{n})$-repr. space of the $d=5 U$-duality group $S O(1,1) \times S O(5, n)$ can be given through the two chains of relations, proceeding (left to right) from 1-charge orbits to 2-charge and then 3 -charge orbits:

$$
\begin{aligned}
& \mathcal{O}_{\frac{1}{2}-B P S, \text { small }, I I} \rightarrow\left\{\begin{aligned}
\mathcal{O}_{\frac{1}{2}-B P S, \text { small }, I} & \rightarrow \mathcal{O}_{\frac{1}{4}-B P S, \text { large }} \\
\mathcal{O}_{n B P S, \text { small }} \rightarrow & \mathcal{O}_{n B P S, \text { large }} \\
\mathcal{O}_{\frac{1}{4}-B P S, \text { small }} & \rightarrow\left\{\begin{array}{l}
\mathcal{O}_{\frac{1}{4}-B P S, \text { large }} \\
\mathcal{O}_{n B P S, \text { large }}
\end{array}\right.
\end{aligned}\right. \\
& \mathcal{O}_{\frac{1}{2}-B P S, \text { small,III }} \rightarrow \mathcal{O}_{\frac{1}{4}-B P S, \text { small }} \rightarrow\left\{\begin{array}{l}
\mathcal{O}_{\frac{1}{4}-B P S, \text { large }} \\
\mathcal{O}_{n B P S, \text { large }}
\end{array}\right.
\end{aligned}
$$

For pure $\mathcal{N}=4, d=5$ supergravity, such a stratification structure simplifies as follows:

$$
\left.\begin{array}{c}
\mathcal{O}_{\frac{1}{2}-B P S, \text { small }, I, n=0} \\
\mathcal{O}_{\frac{1}{2}-B P S, \text { small }, I I I}
\end{array}\right\} \rightarrow \mathcal{O}_{\frac{1}{4}-B P S, \text { large }, n=0} .
$$

Thus, summarizing, $\mathcal{N}=4, d=5$ supergravity theory admits five "small" charge orbits describing the flux configurations supporting static, spherically symmetric, asymptotically flat "small" BHs: one $\frac{1}{4}$-BPS, three $\frac{1}{2}$-BPS and one non-BPS. The "large" orbits are two, namely one $\frac{1}{4}$-BPS and one non-BPS (with $\mathrm{Z}_{A B}=0$ at the horizon).

The relations among the charge orbits of $\mathcal{N}=4, d=5$ supergravity and the charge orbits of $\mathcal{N}=2, d=5$ Jordan symmetric sequence can be determined through the supersymmetry reduction

$$
d=5: \begin{gathered}
\mathcal{N}=4 \\
\mathbb{R} \oplus \boldsymbol{\Gamma}_{5, n}
\end{gathered} \longrightarrow \begin{gathered}
\mathcal{N}=2 \\
\mathbb{R} \oplus \boldsymbol{\Gamma}_{1, n}
\end{gathered},
$$

yielding to the results summarized in Table 6.

Finally, it is worth summarizing the results obtained about the number $\sharp$ of "non-flat" scalar degrees of freedom, within the symmetric RSG studied in previous Sections. For the "magic" supergravities, it holds

$$
J_{3}^{\mathbb{A}}:\left\{\begin{array}{c}
\text { "large" }(\text { rank }=3):\left\{\begin{array}{l}
B P S: \sharp=3 q+2 ; \\
n B P S: \sharp=q+2 ;
\end{array}\right. \\
\text { "small": }\left\{\begin{array}{l}
r a n k=3:\left\{\begin{array}{l}
B P S: \sharp=q+2 \\
n B P S: \sharp=2 ;
\end{array}\right. \\
\text { rank }=3: B P S: \sharp=1,
\end{array}\right.
\end{array}\right.
$$

whereas for $\mathcal{N}=4$ supergravity and $\mathcal{N}=2$ Jordan symmetric sequence the results are reported in Table 6 . As pointed out above, in the symmetric RSG's under consideration the unique scalar degree of freedom on which the ADM mass depends along the 1-charge $\frac{1}{2}$-BPS (maximally symmetric) charge orbits can be interpreted as the Kaluza-Klein radius in the $d=5 \rightarrow d=4$ reduction. 


\begin{tabular}{|c|c|c|}
\hline$r$ & $\overline{\mathcal{N}}=4: \mathbb{R} \oplus \boldsymbol{\Gamma}_{5, n}$ & $\mathcal{N}=2: \mathbb{R} \oplus \boldsymbol{\Gamma}_{1, n}$ \\
\hline 3 & $\begin{array}{c}\mathcal{O}_{\frac{1}{4}-B P S, \text { large }} \\
S O(1,1) \times \frac{S O(5, n)}{S O(4, n)} \\
\sharp=n+1\end{array}$ & $\begin{array}{c}\mathcal{O}_{B P S, \text { large }} \\
{\left[S O(1,1)^{+} \times T_{n}^{+}\right] \cup\left[S O(1,1)^{-} \times T_{n}^{-}\right]} \\
\sharp=n+1 \\
\quad \uparrow * \\
\mathcal{O}_{n B P S, \text { large, } I} \\
{\left[S O(1,1)^{+} \times T_{n}^{-}\right] \cup\left[S O(1,1)^{-} \times T_{n}^{+}\right]} \\
\sharp=n+1\end{array}$ \\
\hline 3 & $\begin{array}{c}\mathcal{O}_{n B P S, \text { large }} \\
S O(1,1) \times \frac{S O(5, n)}{S O(5, n-1)} \\
\sharp=6\end{array}$ & $\begin{array}{c}\mathcal{O}_{n B P S, \text { large }, I I} \\
S O(1,1) \times \frac{S O(1, n)}{S O(1, n-1)} \\
\sharp=2\end{array}$ \\
\hline 2 & $\begin{array}{c}\mathcal{O}_{\frac{1}{2}}-B P S, \text { small,I } \\
S O(1,1) \times \frac{S O(5, n)}{S O(4, n)} \\
\quad \sharp=n+1\end{array}$ & $\begin{array}{l}\mathcal{O}_{B P S, \text { small }, I} \\
S O(1,1) \times T_{n} \\
\quad \sharp=n+1\end{array}$ \\
\hline 2 & $\begin{array}{c}\mathcal{O}_{\frac{1}{4}-B P S, \text { small }} \\
S O(1,1) \times \frac{S O(5, n)}{S O(4, n-1) \rtimes \mathbb{R}^{4, n-1}} \\
\sharp=2\end{array}$ & $\begin{array}{c}\mathcal{O}_{B P S, \text { small,III }} \\
{\left[S O(1,1)^{+} \times \Lambda_{n}^{+}\right] \cup\left[S O(1,1)^{-} \times \Lambda_{n}^{-}\right]} \\
\sharp=2 \\
\quad \begin{array}{r} \\
\end{array} \\
\mathcal{O}_{n B P S, \text { small }, I I} \\
{\left[S O(1,1)^{+} \times \Lambda_{n}^{-}\right] \cup\left[S O(1,1)^{-} \times \Lambda_{n}^{+}\right]} \\
\sharp=2\end{array}$ \\
\hline 2 & $\begin{array}{c}\mathcal{O}_{n B P S, \text { small }} \\
S O(1,1) \times \frac{S O(5, n)}{S O(5, n-1)} \\
\sharp=6\end{array}$ & $\begin{array}{c}\mathcal{O}_{n B P S, \text { small }, I} \\
S O(1,1) \times \frac{S O(1, n)}{S O(1, n-1)} \\
\sharp=2\end{array}$ \\
\hline 1 & $\begin{array}{c}\mathcal{O}_{\frac{1}{2}-B P S, \text { small }, I I} \\
\begin{array}{c}S O(5, n) \\
S O(4, n-1) \times \mathbb{R}^{4, n-1} \\
\sharp=1\end{array}\end{array}$ & $\begin{array}{c}\mathcal{O}_{B P S, \text { small }, I I} \\
S O(1,1) \times \mathbb{R}^{n-1} \\
\sharp=1\end{array}$ \\
\hline 1 & $\begin{array}{c}\mathcal{O}_{\frac{1}{2}-B P S, \text { small }, I I I} \\
S O(1,1) \\
\sharp=1\end{array}$ & $\begin{array}{c}\mathcal{O}_{B P S, \text { small }, I V} \\
S O(1,1) \\
\sharp=1\end{array}$ \\
\hline
\end{tabular}

Table 6: "Large" (rank=3) and "small" (rank=1 and 2) charge orbits of the repr. $(\mathbf{1}, \mathbf{5}+\mathbf{n})$ and $(\mathbf{1}, \mathbf{1}+\mathbf{n})$ of the $d=5 U$-duality groups $S O(1,1) \times S O(5, n)$ and $S O(1,1) \times$ $S O(1, n)$ of $\mathcal{N}=4$ supergravity (based on $\mathbb{R} \oplus \boldsymbol{\Gamma}_{5, n}$ ) and $\mathcal{N}=2$ Jordan symmetric sequence (based on $\mathbb{R} \oplus \boldsymbol{\Gamma}_{1, n}$ ), respectively. The rank $r$ of the orbit is defined as the minimal number of charges defining a representative solution. " $₹ *$ " denotes the fact the orbits are related through a flip of the sign of $q_{H}$. The disconnected timelike hyperboloid $T_{n}$ and lightcone $\Lambda_{n}$ structures are defined by $(6.2)$ and $(6.3)$, respectively. $\sharp$, defined in (4.3), denotes the number of "non-flat" scalar degrees of freedom supported by the charge orbit 


\section{Acknowledgments}

S. F. and A. M. would like to thank L. Borsten and M. Trigiante for enlightening discussions.

B. L. C. and A. M. would like to thank the CTP of the University of California, Berkeley, CA USA, where part of this work was done, for kind hospitality and stimulating environment. A. M. would also like to acknowledge the warm hospitality and inspiring environment of the Department of Physics, Theory Unit Group at CERN, Geneva CH.

The work of B. L. C. has been supported in part by the European Commission under the FP7-PEOPLE-IRG-2008 Grant n PIRG04-GA-2008-239412 "String Theory and Noncommutative Geometry" (STRING).

The work of S. F. has been supported in part by the ERC Advanced Grant no. 226455, "Supersymmetry, Quantum Gravity and Gauge Fields" (SUPERFIELDS), and also in part by INFN - Frascati National Laboratories, and by D.O.E. grant DE-FG03-91ER40662, Task C.

The work of A. M. has been supported by an INFN visiting Theoretical Fellowship at SITP, Stanford University, Stanford, CA, USA.

The work of B. Z. has been supported in part by the Director, Office of Science, Office of High Energy and Nuclear Physics, Division of High Energy Physics of the U.S. Department of Energy under Contract No. DE-AC02-05CH11231, and in part by NSF grant 10996-13607-44 PHHXM.

\section{A Resolution of $G_{5}$-invariant Constraints}

In this Appendix, we explicitly solve the $G_{5}$-invariant defining constraints of "small" charge orbits in "magic" symmetric RSG, both in the "bare" (Sub-App. A.1) and "dressed" (Sub-App. A.2) charges bases.

\section{A.1 "Bare" Charges Basis}

Let us start by noticing that for each of the four "magic" symmetric RSG's a unique maximal symmetric embedding into $G_{5}$ exists containing a factor $S O(1,1)$. It reads (recall Eq. (4.30) [54]

$$
G_{5} \supsetneq \max G_{6} \times \mathcal{A}_{q} \times S O(1,1),
$$

where the group $\mathcal{A}_{q}$ has been defined in Table 2 . Notice that, in the cases $q=4$ and $2, G_{6} \times S O(1,1)$ is not embedded maximally (also considering non-symmetric embeddings [55]) into $G_{5}$ itself.

When removing $\mathcal{A}_{q}$ in the cases $q=4$ and 2 (and thus losing the maximality), the embedding A.1) has a nice interpretation in terms of truncation of the "magic" supergravity to theories belonging to the Jordan symmetric sequence (3.44) [19]:

$$
\begin{array}{ll}
J_{3}^{\mathbb{Q}} \supsetneq \max \mathbb{R} \oplus J_{2}^{\mathbb{Q}}: & E_{6(-26) \supsetneq \max } S O(1,1) \times S O(1,9) ; \\
J_{3}^{\mathbb{H}} \supsetneq \mathbb{R} \oplus J_{2}^{\mathbb{H}}: & S U^{*}(6) \supsetneq S O(1,1) \times S O(1,5) ; \\
J_{3}^{\mathbb{C}} \supsetneq \mathbb{R} \oplus J_{2}^{\mathbb{C}}: & S L(3, \mathbb{C}) \supsetneq S O(1,1) \times S O(1,3) ; \\
J_{3}^{\mathbb{R}} \supsetneq \max \mathbb{R} \oplus J_{2}^{\mathbb{R}}: & S L(3, \mathbb{R}) \supsetneq \max S O(1,1) \times S O(1,2),
\end{array}
$$

where it should be recalled that $(q=8,4,2,1$; see e.g. [47])

$$
J_{2}^{\mathbb{A}} \sim \boldsymbol{\Gamma}_{1, q+1} .
$$




\section{A.1.1 $\mathcal{O}_{\text {lightlike }, B P S}$}

In order to solve the "small" lightlike $G_{5}$-invariant defining constraints 4.51 in "bare" charges in a way consistent with an orbit representative having $Z \neq 0$, let us further embed the $m c s$ of the group in the right-hand side of Eq. A.1, thus obtaining

$$
G_{5} \supsetneq \max G_{6} \times \mathcal{A}_{q} \times S O(1,1) \stackrel{m c s}{\supsetneq} S O(q+1) \times \mathcal{A}_{q} .
$$

Thus, under the "branching" (A.4) the irrepr. $\mathbf{R}_{Q}$ of $G_{5}$ in which the electric charges $q_{i}$ 's sit decomposes as follows:

$$
\begin{aligned}
\mathbf{R}_{Q} & \rightarrow(\mathbf{1}, \mathbf{1})_{+4}+(\mathbf{q}+\mathbf{2}, \mathbf{1})_{-2}+\left(\operatorname{Spin}(q+2), \operatorname{Spin}\left(Q_{q}\right)\right)_{+1} \\
& \rightarrow(\mathbf{1}, \mathbf{1})_{I}+(\mathbf{q}+\mathbf{1}, \mathbf{1})+(\mathbf{1}, \mathbf{1})_{I I}+\left(\operatorname{Spin}(q+1), \operatorname{Spin}\left(Q_{q}\right)\right) .
\end{aligned}
$$

This in turn entails the "branching"

$$
q_{i} \longrightarrow\left(q_{(\mathbf{1}, \mathbf{1})_{I}}, q_{(\mathbf{1}, \mathbf{1})_{I I}}, q_{(\mathbf{q}+\mathbf{1}, \mathbf{1})}, q_{\left(\mathbf{S p i n}(q+1), \operatorname{Spin}\left(Q_{q}\right)\right)}\right) .
$$

In the first line of A.5 subscripts denote the weight with respect to $S O(1,1)$, whereas in the second line they just discriminate between the two singlets of $S O(q+1) \times \mathcal{A}_{q}$. Also recall that, as given in Table $2, \mathcal{A}_{q}$ and $Q_{q}$ are absent for $q=8$ and $q=1$.

Therefore, with respect to $S O(q+1) \times \mathcal{A}_{q}$, one obtains:

- two singlets (note that $(\mathbf{1}, \mathbf{1})_{I}$ is a singlet of $S O(q+1,1) \times \mathcal{A}_{q}$, as well);

- one vector $(\mathbf{q}+\mathbf{1}, \mathbf{1})$;

- a (double-) spinor $\left(\operatorname{Spin}(q+1), \operatorname{Spin}\left(Q_{q}\right)\right)$.

The representation decomposition A.5 yields that $d^{i j k}$, the rank-3 completely symmetric $G_{5^{-}}$ invariant tensor (namely, the unique singlet in the tensor product $\left(\mathbf{R}_{Q}\right)^{3}$ ) decomposes in a such way that $(\mathbf{1}, \mathbf{1})_{I I}$ and $(\mathbf{q}+\mathbf{1}, \mathbf{1})$ have the same couplings inside $\left(\mathbf{R}_{Q}\right)^{3}$.

Details concerning the various "magic" symmetric RSG's are given further below.

The position which solves (with maximal - compact - symmetry $\left.S O(q+1) \times \mathcal{A}_{q}\right)$ the "small" lightlike $G_{5}$-invariant defining constraints (4.51) in "bare" charges (and in a way consistent with an orbit representative having $Z \neq 0$ ) reads as follows:

$$
\left\{\begin{array}{l}
q_{(\mathbf{1}, \mathbf{1})_{I}}=0 \\
q_{(\mathbf{q}+\mathbf{1}, \mathbf{1})}=0 \\
q_{\left(\mathbf{S p i n}(q+1), \operatorname{Spin}\left(Q_{q}\right)\right)}=0 \\
q_{(\mathbf{1}, \mathbf{1})_{I I}} \neq 0
\end{array}\right.
$$

Since $S O(q+1) \times \mathcal{A}_{q}$ is the unique group maximally (and symmetrically) embedded into $G_{6} \times \mathcal{A}_{q} \times$ $S O(1,1)$ which has $S O(q+1) \times \mathcal{A}_{q}$ as (in this case improper) $m c s$, it follows that $S O(q+1) \times \mathcal{A}_{q}$ is also the maximal semi-simple symmetry of $\mathcal{O}_{\text {lightlike,BPS }}$, which is thus given by Eq. (4.14).

The origin of the non-semi-simple Abelian (namely, translational) factor $\mathbb{R}^{\left(\operatorname{spin}(q+1), \operatorname{spin}\left(Q_{q}\right)\right)}$ in the stabilizer of $\mathcal{O}_{\text {lightlike,BPS }}$ will be explained through the procedure of suitable İnönü-Wigner contraction performed in Sub-App. B.1. 


\section{A.1.2 $\mathcal{O}_{\text {critical }, B P S}$}

Eq. A.4 and subsequent ones are also relevant for the resolution of the "small" critical $G_{5^{-}}$ invariant defining constraints 4.55) in "bare" charges in a way consistent with an orbit representative having $Z \neq 0$ (which is the unique possible case; see treatment above). In this case, the position which solves (with maximal - non-compact - symmetry $G_{6} \times \mathcal{A}_{q}$ ) the constraints (4.55) in "bare" charges reads as follows:

$$
\left\{\begin{array}{l}
q_{(\mathbf{1}, \mathbf{1})_{I I}}=0 ; \\
q_{(\mathbf{q}+\mathbf{1}, \mathbf{1})}=0 ; \\
q_{\left(\mathbf{S p i n}(q+1), \operatorname{Spin}\left(Q_{q}\right)\right)}=0 ; \\
q_{(\mathbf{1}, \mathbf{1})_{I}} \neq 0 .
\end{array}\right.
$$

At least for the relevant values $q=8,4,2,1$ it holds that $\operatorname{spin}(q+2)=\operatorname{spin}(q+1)$ (recall definition (4.17)). Therefore, since

$$
\left.\begin{array}{rl}
q_{(\mathbf{1}, \mathbf{1})_{I I}} & =0 ; \\
q_{(\mathbf{q}+\mathbf{1}, \mathbf{1})} & =0 ;
\end{array}\right\} \Leftrightarrow q_{(\mathbf{q}+\mathbf{2}, \mathbf{1})}=0,
$$

it follows that the position (A.8) exhibits maximal - non-compact - symmetry $G_{6} \times \mathcal{A}_{q}$, which then is the maximal semi-simple symmetry of $\mathcal{O}_{\text {critical,BPS }}$, which is thus given by Eq. 4.29 .

The origin of $\mathbb{R}^{\left(\operatorname{spin}(q+2), \operatorname{spin}\left(Q_{q}\right)\right)}$ in the stabilizer of $\mathcal{O}_{\text {critical,BPS }}$ will be explained through the procedure of suitable $S O(1,1)$-(three-)grading performed in Sub-App. B.2.

\section{A.1.3 $\mathcal{O}_{\text {lightlike, } n B P S}$}

In order to solve the "small" lightlike $G_{5}$-invariant defining constraints 4.51 in "bare" charges in a way consistent with an orbit representative having $Z=0$, the embedding (A.1) has to be further elaborated as follows:

$$
G_{5} \supsetneq \max G_{6} \times \mathcal{A}_{q} \times S O(1,1) \supsetneq \max S O(q, 1) \times \mathcal{A}_{q} \times S O(1,1) \stackrel{m c s}{\supsetneq} S O(q) \times \mathcal{A}_{q} .
$$

Thus, under the "branching" A.10 the irrepr. $\mathbf{R}_{Q}$ decomposes as follows :

$$
\begin{aligned}
\mathbf{R}_{Q} & \rightarrow(\mathbf{1}, \mathbf{1})_{+4}+(\mathbf{q}+\mathbf{2}, \mathbf{1})_{-2}+\left(\operatorname{Spin}(q+2), \operatorname{Spin}\left(Q_{q}\right)\right)_{+1} \\
& \rightarrow(\mathbf{1}, \mathbf{1})_{+4}+(\mathbf{q}+\mathbf{1}, \mathbf{1})_{-2}+(\mathbf{1}, \mathbf{1})_{-2}+\left(\operatorname{Spin}(q+1), \operatorname{Spin}\left(Q_{q}\right)\right)_{+1} \\
& \rightarrow(\mathbf{1}, \mathbf{1})_{I}+(\mathbf{q}, \mathbf{1})+(\mathbf{1}, \mathbf{1})_{I I I}+(\mathbf{1}, \mathbf{1})_{I I}+\left(\mathbf{S p i n}^{\prime}(q), \operatorname{Spin}\left(Q_{q}\right)\right)+\left(\operatorname{Spin}^{\prime \prime}(q), \operatorname{Spin}\left(Q_{q}\right)\right),
\end{aligned}
$$

where, besides the obvious irrepr. decompositions determining the last line of (A.11), one should recall that

$$
\left(\operatorname{Spin}(q+1), \operatorname{Spin}\left(Q_{q}\right)\right) \longrightarrow\left(\operatorname{Spin}^{\prime}(q), \operatorname{Spin}\left(Q_{q}\right)\right)+\left(\operatorname{Spin}^{\prime \prime}(q), \operatorname{Spin}\left(Q_{q}\right)\right),
$$

where the primes discriminate between the two spinor irreprs. of $S O(q) \times \mathcal{A}_{q}$. The "branching" of electric charges corresponding to A.11) reads

$$
q_{i} \longrightarrow\left(q_{(\mathbf{1}, \mathbf{1})_{I}}, q_{(\mathbf{1}, \mathbf{1})_{I I}}, q_{(\mathbf{1}, \mathbf{1})_{I I I}}, q_{(\mathbf{q}, \mathbf{1})}, q_{\left(\mathbf{S p i n}^{\prime}(q), \operatorname{Spin}\left(Q_{q}\right)\right)}, q_{\left(\mathbf{S p i n}^{\prime \prime}(q), \operatorname{Spin}\left(Q_{q}\right)\right)}\right) .
$$

In the first and second line of A.11) subscripts denote the weight with respect to $S O(1,1)$, whereas in the third line they just discriminate between the three singlets of $S O(q) \times \mathcal{A}_{q}$.

Therefore, with respect to $S O(q) \times \mathcal{A}_{q}$, one obtains: 
- three singlets (notice that $(\mathbf{1}, \mathbf{1})_{I}$ is also singlet of $S O(q, 1) \times \mathcal{A}_{q}$ and of $G_{6} \times \mathcal{A}_{q}$, and that $(\mathbf{1}, \mathbf{1})_{I I}$ is singlet of $S O(q, 1) \times \mathcal{A}_{q}$, as well $)$;

- a vector $(\mathbf{q}, \mathbf{1})$;

- two (double-)spinors $\left(\operatorname{Spin}^{\prime}(q), \operatorname{Spin}\left(Q_{q}\right)\right)$ and $\left(\operatorname{Spin}^{\prime \prime}(q), \operatorname{Spin}\left(Q_{q}\right)\right)$.

As a feature peculiar to $\mathrm{A.11}$, the vector $(\mathbf{q}, \mathbf{1})$ and the two (double-) spinors $\left(\mathbf{S p i n}^{\prime}(q), \mathbf{S p i n}\left(Q_{q}\right)\right)$ and $\left(\mathbf{S p i n}^{\prime \prime}(q), \mathbf{S p i n}\left(Q_{q}\right)\right)$ do exhibit a "triality symmetry", realized differently depending on $q=8,4,2,1$, as given in Sub-App. A.1.

The representation decomposition A.11 yields that $d^{i j k}$ decomposes in such a way that the manifest "triality" exhibited by the "branching" of $\mathbf{R}_{Q}$ is removed, and the two (double-)spinors are put on a different footing with respect to the vector. As a consequence:

- $(\mathbf{1}, \mathbf{1})_{I I},(\mathbf{1}, \mathbf{1})_{I I I}$ and $(\mathbf{q}, \mathbf{1})$;

- $\left(\operatorname{Spin}^{\prime}(q), \operatorname{Spin}\left(Q_{q}\right)\right)$ and $\left(\operatorname{Spin}^{\prime \prime}(q), \operatorname{Spin}\left(Q_{q}\right)\right)$

separately have the same couplings inside $\left(\mathbf{R}_{Q}\right)^{3}$.

The position which solves (with maximal - compact - symmetry $S O(q) \times \mathcal{A}_{q}$ ) the "small" lightlike $G_{5}$-invariant defining constraints (4.51) in "bare" charges (and in a way consistent with an orbit representative having $Z=0$ ) reads as follows:

$$
\left\{\begin{array}{l}
q_{(\mathbf{q}, \mathbf{1})}=0 \\
q_{\left(\mathbf{S p i n}^{\prime}(q), \mathbf{S p i n}\left(Q_{q}\right)\right)}=0 \\
q_{\left(\mathbf{S p i n}^{\prime \prime}(q), \operatorname{Spin}\left(Q_{q}\right)\right)}=0
\end{array}\right.
$$

with the three singlets $q_{(\mathbf{1}, \mathbf{1})_{I}}, q_{(\mathbf{1}, \mathbf{1})_{I I}}$ and $q_{(\mathbf{1}, \mathbf{1})_{I I I}}$ constrained by

$$
q_{(\mathbf{1}, \mathbf{1})_{I}}\left[\begin{array}{l}
d_{(\mathbf{1}, \mathbf{1})_{I}(\mathbf{1}, \mathbf{1})_{I I}(\mathbf{1}, \mathbf{1})_{I I}} q_{(\mathbf{1}, \mathbf{1})_{I I}}^{2} \\
+2 d_{(\mathbf{1}, \mathbf{1})_{I}(\mathbf{1}, \mathbf{1})_{I I}(\mathbf{1}, \mathbf{1})_{I I I}} q_{(\mathbf{1}, \mathbf{1})_{I I}} q_{(\mathbf{1}, \mathbf{1})_{I I I}} \\
+d_{(\mathbf{1}, \mathbf{1})_{I}(\mathbf{1}, \mathbf{1})_{I I I}(\mathbf{1}, \mathbf{1})_{I I I}} q_{(\mathbf{1}, \mathbf{1})_{I I I}}^{2}
\end{array}\right]=0 .
$$

Notice that in (A.14) the charges related to the vector and to the two (double-)spinors are on equal footing, thus exhibiting a "triality symmetry", as already mentioned above.

Notice that $S O(q, 1) \times \mathcal{A}_{q}$ is the unique group which is maximally (if one consider also the factor $S O(1,1))$ and symmetrically embedded into $G_{6} \times \mathcal{A}_{q} \times S O(1,1)$, and also which has $S O(q) \times \mathcal{A}_{q}$ as $m c s$. Therefore, it follows that $S O(q, 1) \times \mathcal{A}_{q}$ is also the maximal semi-simple symmetry of $\mathcal{O}_{\text {lightlike, } n B P S}$, which is thus given by Eq. (4.24).

As mentioned above, the origin of $\mathbb{R}^{\left(\operatorname{spin}(q+1), \operatorname{spin}\left(Q_{q}\right)\right)}$ in the stabilizer of $\mathcal{O}_{\text {lightlike, } n B P S}$ will be explained through the procedure of suitable İnönü-Wigner contraction performed in Sub-App. B.1.

\section{A.1.4 Details}

We now explicit some details of the treatment of symmetric "magic" RSG.

We start by giving the explicit form of Eqs. A.4 and A.5 for all $q=8,4,2,1$ classifying symmetric "magic" RSG. 
- $q=8\left(J_{3}^{\mathbb{Q}}\right)$

$$
\begin{aligned}
& E_{6(-26)} \supsetneq \max S O(9,1) \times S O(1,1) \stackrel{m c s}{\supsetneq} S O(9) ; \\
& \mathbf{2 7} \rightarrow \mathbf{1}_{+4}+\mathbf{1 0}_{-2}+\mathbf{1 6}_{+1} \rightarrow \mathbf{1}_{I}+\mathbf{9}+\mathbf{1}_{I I}+\mathbf{1 6} .
\end{aligned}
$$

- $q=4\left(J_{3}^{\mathbb{H}}\right)\left(S O(5,1) \sim S U^{*}(4), S O(5) \sim U S p(4)\right)$

$$
\begin{aligned}
& S U^{*}(6) \supsetneq \max S O(5,1) \times S O(3) \times S O(1,1) \stackrel{m c s}{\supsetneq} S O(5) \times S O(3) ; \\
& \mathbf{1 5} \rightarrow(\mathbf{1}, \mathbf{1})_{+4}+(\mathbf{6}, \mathbf{1})_{-2}+(\mathbf{4}, \mathbf{2})_{+1} \rightarrow(\mathbf{1}, \mathbf{1})_{I}+(\mathbf{5}, \mathbf{1})+(\mathbf{1}, \mathbf{1})_{I I}+(\mathbf{4}, \mathbf{2}) .
\end{aligned}
$$

- $q=2\left(J_{3}^{\mathbb{C}}\right)(S L(2, \mathbb{C}) \sim S O(3,1), G L(1, \mathbb{C}) \sim S O(2) \times S O(1,1))$

$$
\begin{aligned}
& S L(3, \mathbb{C}) \supsetneq \max S L(2, \mathbb{C}) \times S L(1, \mathbb{C}) \times G L(1, \mathbb{C}) \stackrel{m c s}{\supsetneq} S O(3) \times S O(2) ; \\
& \mathbf{9} \rightarrow\left(\mathbf{1}_{0}\right)_{+4}+\left(\mathbf{3}_{0}+\mathbf{1}_{0}\right)_{-2}+\left(\mathbf{2}_{3}+\overline{\mathbf{2}}_{-3}\right)_{+1} \rightarrow\left(\mathbf{1}_{0}\right)_{I}+\mathbf{3}_{0}+\left(\mathbf{1}_{0}\right)_{I I}+\mathbf{2}_{3}+\mathbf{2}_{-3},
\end{aligned}
$$

where the first subscript in the second step and the subscript in the last step denote charges w.r.t. $S O(2) \sim U(1)$, as well as the second subscript in the second step denotes weights w.r.t. $S O(1,1)$. In order to derive $($ A.18), the decompositions of the irreprs. of $S L(3, \mathbb{C})$ under $S L(2, \mathbb{C}) \times S L(1, \mathbb{C}) \times G L(1, \mathbb{C}) \sim S L(2, \mathbb{C}) \times S O(2) \times S O(1,1)$ have been recalled (the charges and weights w.r.t. $S O(2)$ and $S O(1,1)$ are given):

$$
\begin{gathered}
3 \rightarrow(2,1,-1)+(1,-2,2) \\
\overline{3} \rightarrow(\overline{2},-1,-1)+(1,2,2) ; \\
3^{\prime} \rightarrow(2,-1,1)+(1,2,-2) \\
\overline{3}^{\prime} \rightarrow(\overline{2}, \mathbf{1}, \mathbf{1})+(\mathbf{1},-2,-2) .
\end{gathered}
$$

Thus, through A.19 and A.20, the irrepr.

$$
\mathbf{R}_{q=2}=\mathbf{9} \equiv \mathbf{3} \times \overline{\mathbf{3}}
$$

branches as given by A.18.

- $q=1\left(J_{3}^{\mathbb{R}}\right)(S L(2, \mathbb{R}) \sim S O(2,1))$

$$
\begin{aligned}
& S L(3, \mathbb{R}) \supsetneq \max S O(2,1) \times S O(1,1) \stackrel{m c s}{\supsetneq} S O(2) ; \\
& \mathbf{6}^{\prime} \rightarrow \mathbf{1}_{+4}+\mathbf{3}_{-2}+\mathbf{2}_{+1} \rightarrow \mathbf{1}_{I}+\mathbf{2}+\mathbf{1}_{I I}+\mathbf{2},
\end{aligned}
$$

where the the normalizations and conventions of Table 58 of [55] have been adopted.

Next, we explicit Eqs. A.10 and A.11 for all $q=8,4,2,1$ classifying symmetric "magic" RSG. 
- $q=8\left(J_{3}^{\mathbb{Q}}\right)$

$$
\begin{aligned}
& E_{6(-26)} \supsetneq \max S O(9,1) \times S O(1,1) \supsetneq \max S O(8,1) \times S O(1,1) \stackrel{m c s}{\supsetneq} S O(8) \\
& \mathbf{2 7} \rightarrow \mathbf{1}_{+4}+\mathbf{1 0}_{-2}+\mathbf{1 6}_{+1} \rightarrow \mathbf{1}_{+4}+\mathbf{9}_{-2}+\mathbf{1}_{-2}+\mathbf{1 6}_{+1} \rightarrow \mathbf{1}_{I}+\mathbf{8}_{v}+\mathbf{1}_{I I I}+\mathbf{1}_{I I}+\mathbf{8}_{s}+\mathbf{8}_{c} .
\end{aligned}
$$

The "triality" in irreprs. of $S O(q)$ is here implemented through the triality of $\left(\mathbf{8}_{v}, \mathbf{8}_{s}, \mathbf{8}_{c}\right)$ of $S O(8)$.

- $q=4\left(J_{3}^{\mathbb{H}}\right)$

$$
\begin{aligned}
& S U^{*}(6) \supsetneq \max S O(5,1) \times S O(3) \times S O(1,1) \\
& \supsetneq_{\max } S O(4,1) \times S O(3) \times S O(1,1) \\
& \stackrel{m c s}{\supsetneq} S O(4) \times S O(3) \sim S U(2) \times S U(2) \times S U(2) \text {; } \\
& 15 \rightarrow(\mathbf{1}, \mathbf{1})_{+4}+(\mathbf{6}, \mathbf{1})_{-2}+(\mathbf{4}, \mathbf{2})_{+1} \rightarrow(\mathbf{1}, \mathbf{1})_{+4}+(\mathbf{5}, \mathbf{1})_{-2}+(\mathbf{1}, \mathbf{1})_{-2}+(\mathbf{4}, \mathbf{2})_{+1} \rightarrow \\
& \rightarrow(\mathbf{1}, \mathbf{1}, \mathbf{1})_{I}+(\mathbf{2}, \mathbf{2}, \mathbf{1})+(\mathbf{1}, \mathbf{1}, \mathbf{1})_{I I I}+(\mathbf{1}, \mathbf{1}, \mathbf{1})_{I I}+(\mathbf{1}, \mathbf{2}, \mathbf{2})+(\mathbf{2}, \mathbf{1}, \mathbf{2}) \text {. }
\end{aligned}
$$

Thus, the "triality" in irreprs. of $S O(q) \times \mathcal{A}_{q}$ is implemented for $q=4$ through the triality of $((\mathbf{2}, \mathbf{2}, \mathbf{1}),(\mathbf{2}, \mathbf{1}, \mathbf{2}),(\mathbf{1}, \mathbf{2}, \mathbf{2}))$ of $S U(2) \times S U(2) \times S U(2)$.

- $q=2\left(J_{3}^{\mathbb{C}}\right)$

$$
\begin{aligned}
& S L(3, \mathbb{C}) \supsetneq \max S L(2, \mathbb{C}) \times S L(1, \mathbb{C}) \times G L(1, \mathbb{C}) \\
& \supsetneq \max S O(2,1) \times S O(2) \times S O(1,1) \stackrel{m c s}{\supsetneq} S O(2) \times S O(2) ; \quad \text { (A.28) } \\
& \mathbf{9} \rightarrow\left(\mathbf{1}_{0}\right)_{+4}+\left(\mathbf{3}_{0}+\mathbf{1}_{0}\right)_{-2}+\left(\mathbf{2}_{3}+\overline{\mathbf{2}}_{-3}\right)_{+1} \rightarrow\left(\mathbf{1}_{0}\right)_{+4}+\left(\mathbf{3}_{0}\right)_{-2}+\left(\mathbf{1}_{0}\right)_{-2}+\left(\mathbf{2}_{3}\right)_{+1}+\left(\mathbf{2}_{-3}\right)_{+1} \\
& \quad \rightarrow\left(\mathbf{1}_{0}\right)_{I}+\mathbf{2}_{0}+\left(\mathbf{1}_{0}\right)_{I I I}+\left(\mathbf{1}_{0}\right)_{I I}+\mathbf{2}_{3}+\mathbf{2}_{-3} .
\end{aligned}
$$

Thus, the triality in irreprs. of $S O(q) \times \mathcal{A}_{q}$ is implemented for $q=2$ through the triality of $\left(\mathbf{2}_{0}, \mathbf{2}_{3}, \mathbf{2}_{-3}\right)$ of $S O(2) \times S O(2)$ (notice the different charges w.r.t. $\left.\mathcal{A}_{q=2}=S O(2) \sim U(1)\right)$.

- $q=1\left(J_{3}^{\mathbb{R}}\right)$

$$
\begin{aligned}
& S L(3, \mathbb{R}) \supsetneq \max S O(2,1) \times S O(1,1) \supsetneq \max S O(1,1) \times S O(1,1) \stackrel{m c s}{\supsetneq} 1 ; \\
& \mathbf{6}^{\prime} \rightarrow \mathbf{1}_{+4}+\mathbf{3}_{-2}+\mathbf{2}_{+1} \rightarrow \mathbf{1}_{+4}+\mathbf{2}_{-2}+\mathbf{1}_{-2}+\mathbf{2}_{+1} \rightarrow \mathbf{1}_{I}+\mathbf{1}_{I I}+\mathbf{1}_{I I I}+\mathbf{1}_{I V}+\mathbf{1}_{V}+\mathbf{1}_{V I},
\end{aligned}
$$

where in the first line 1 denotes the identity element. Notice that there is no compact symmetry in $\mathcal{O}_{\text {lightlike }, n B P S, J_{3}^{\mathbb{R}}, d=5}$, as also given by the fact that $\mathcal{M}_{\text {lightlike, } n B P S, J_{3}^{\mathbb{R}}, d=5}=$ $S O(1,1) \rtimes \mathbb{R}^{2}$ (see Table 3 ). Thus, the "triality" of irreprs. of $S O(q)$ in this case trivially degenerates into a "sextality" (six singlets in the r.h.s. of the second line of A.30). 


\section{A.2 "Dressed" Charges Basis}

Concerning the resolution of the $G_{5}$-invariant (sets of) constraints in the basis of "dressed" charges, one should notice that for each of the four "magic" symmetric RSG's a unique non-compact, real form $\widetilde{H}_{5}$ of the compact group $H_{5} \equiv m c s\left(G_{5}\right)$ exists with maximal symmetric embedding into $G_{5}$ (see e.g. [54]; also recall Subsect. 3.4 and Table 1):

$$
G_{5} \supsetneq \max \widetilde{H}_{5} \text {. }
$$

\section{A.2.1 $\mathcal{O}_{\text {lightlike }, B P S}$}

In order to solve the "small" lightlike $G_{5}$-invariant defining constraints (4.51) in "dressed" charges in a way consistent with an orbit representative with $Z \neq 0$, let us further embed

$$
\widetilde{h}_{5} \equiv m c s\left(\widetilde{H}_{5}\right)=S O(q+1) \times \mathcal{A}_{q},
$$

thus obtaining

$$
G_{5}\left(\supsetneq \max \widetilde{H}_{5}\right) \stackrel{m c s}{\supsetneq} S O(q+1) \times \mathcal{A}_{q},
$$

where the brackets denote the auxiliary nature of the embedding. Thus, under the "branching" A.33 $\mathbf{R}_{Q}$ decomposes as follows:

$$
\mathbf{R}_{Q}(\rightarrow \mathbf{1}+\widehat{\mathbf{R}}) \rightarrow(\mathbf{1}, \mathbf{1})_{I}+(\mathbf{q}+\mathbf{1}, \mathbf{1})+\left(\operatorname{Spin}(q+1), \operatorname{Spin}\left(Q_{q}\right)\right)+(\mathbf{1}, \mathbf{1})_{I I},
$$

where $\widehat{\mathbf{R}}$ is an irrepr. of $\widetilde{H}_{5}$ used as an intermediate step. Eq. A.34 corresponds to the "branching"

$$
\mathcal{Z} \equiv\left(Z, Z_{x}\right) \longrightarrow\left(Z, Z_{(\mathbf{1}, \mathbf{1})_{I I}}, Z_{(\mathbf{q}+\mathbf{1}, \mathbf{1})}, Z_{\left(\operatorname{Spin}(q+1), \operatorname{Spin}\left(Q_{q}\right)\right)}\right)
$$

where

$$
\mathcal{Z}_{(\mathbf{1}, \mathbf{1})_{I}} \equiv Z
$$

throughout. Therefore, with respect to $S O(q+1) \times \mathcal{A}_{q}$, one obtains:

- two singlets;

- one vector $(\mathbf{q}+\mathbf{1}, \mathbf{1})$;

- one (double-)spinor $\left(\mathbf{S p i n}(q+1), \operatorname{Spin}\left(Q_{q}\right)\right)$.

The position which solves (with maximal - compact - symmetry $\left.S O(q+1) \times \mathcal{A}_{q}\right)$ the "small" lightlike $G_{5}$-invariant defining constraints $(4.51)$ in "dressed" charges (and in a way consistent with an orbit representative having $Z \neq 0$ ) reads as follows:

$$
\left\{\begin{array}{l}
Z_{(\mathbf{q}+\mathbf{1}, \mathbf{1})}=0 \\
Z_{\left(\mathbf{S p i n}(q+1), \operatorname{Spin}\left(Q_{q}\right)\right)}=0,
\end{array}\right.
$$

with $Z$ and $Z_{(\mathbf{1}, \mathbf{1})_{I I}}$ constrained by:

$$
Z^{3}-\left(\frac{3}{2}\right)^{2} Z Z_{(\mathbf{1}, \mathbf{1})_{I I}}^{2}-\left(\frac{3}{2}\right)^{\frac{3}{2}} T_{(\mathbf{1}, \mathbf{1})_{I I}(\mathbf{1}, \mathbf{1})_{I I}(\mathbf{1}, \mathbf{1})_{I I}} Z_{(\mathbf{1}, \mathbf{1})_{I I}}^{3}=0 .
$$


Notice that $S O(q+1) \times \mathcal{A}_{q}$ is the unique group which is maximally (and symmetrically) embedded into $\widetilde{H}_{5}$ and which has $S O(q+1) \times \mathcal{A}_{q}$ as (in this case improper) $m c s$ (actually, $S O(q+1) \times \mathcal{A}_{q}=$ $\left.m c s\left(\widetilde{H}_{5}\right)\right)$. Therefore, it follows that $S O(q+1) \times \mathcal{A}_{q}$ is also the maximal semi-simple symmetry of $\mathcal{O}_{\text {lightlike, } B P S}$, which is thus given by Eq. 4.14.

The explicit form of Eqs. A.33 and A.34 for all $q=8,4,2,1$ classifying symmetric "magic" RSG is given below.

- $q=8\left(J_{3}^{\mathbb{Q}}\right)$

$$
\begin{aligned}
& E_{6(-26)}\left(\supsetneq \max F_{4(-20)}\right) \stackrel{m c s}{\supsetneq} S O(9) ; \\
& \mathbf{2 7}(\rightarrow \mathbf{1}+\mathbf{2 6}) \rightarrow \mathbf{1}_{I}+\mathbf{9}+\mathbf{1 6}+\mathbf{1}_{I I} .
\end{aligned}
$$

- $q=4\left(J_{3}^{\mathbb{H}}\right)$

$$
\begin{aligned}
& S U^{*}(6)(\supsetneq \max U S p(4,2)) \stackrel{m c s}{\supsetneq} U S p(4) \times U S p(2) \sim S O(5) \times S O(3) ; \\
& \mathbf{1 5}(\rightarrow \mathbf{1}+\mathbf{1 4}) \rightarrow(\mathbf{1}, \mathbf{1})_{I}+(\mathbf{5}, \mathbf{1})+(\mathbf{4}, \mathbf{2})+(\mathbf{1}, \mathbf{1})_{I I} .
\end{aligned}
$$

- $q=2\left(J_{3}^{\mathbb{C}}\right)$

$$
\begin{aligned}
& S L(3, \mathbb{C})(\supsetneq \max S U(2,1)) \stackrel{m c s}{\supsetneq} S U(2) \times U(1) \sim S O(3) \times S O(2) ; \\
& \mathbf{9}(\rightarrow \mathbf{1}+\mathbf{8}) \rightarrow\left(\mathbf{1}_{0}\right)_{I}+\mathbf{2}_{-3}+\mathbf{2}_{3}+\mathbf{3}_{0}+\left(\mathbf{1}_{0}\right)_{I I} .
\end{aligned}
$$

- $q=1\left(J_{3}^{\mathbb{R}}\right)$

$$
\begin{aligned}
& S L(3, \mathbb{R})(\supsetneq \max S O(2,1)) \stackrel{m c s}{\supsetneq} S O(2) ; \\
& \mathbf{6}^{\prime}(\rightarrow \mathbf{1}+\mathbf{5}) \rightarrow \mathbf{1}_{I}+\mathbf{2}+\mathbf{2}+\mathbf{1}_{I I} .
\end{aligned}
$$

As mentioned in the resolution in the basis of "bare" (electric) charges $q_{i}$ 's, the origin of $\mathbb{R}^{\left(\operatorname{spin}(q+1), \operatorname{spin}\left(Q_{q}\right)\right)}$ in the stabilizer of $\mathcal{O}_{\text {lightlike,BPS }}$ will be explained through the procedure of suitable İnönü-Wigner contraction performed in Sub-App. B.1.

\section{A.2.2 $\mathcal{O}_{\text {lightlike, } n B P S}$}

In order to solve the "small" lightlike $G_{5}$-invariant defining constraints (4.51) in "dressed" charges in a way consistent with an orbit representative having $Z=0$, the embedding (A.31) has to be further elaborated as follows:

$$
G_{5}\left(\supsetneq \max \widetilde{H}_{5}\right) \supsetneq \max \widehat{h}_{5} \stackrel{m c s}{\supsetneq} S O(q) \times \mathcal{A}_{q},
$$

where

$$
\widehat{h}_{5}=S O(q, 1) \times \mathcal{A}_{q}
$$

is the unique non-compact form of $\widetilde{h}_{5}$ (defined by A.32) to be embedded maximally and symmetrically into $\widetilde{H}_{5}$ (see e.g. [54]). 
Thus, under the "branching" A.43 $\mathbf{R}_{Q}$ decomposes as follows:

$$
\begin{aligned}
& \mathbf{R}_{Q}(\rightarrow \mathbf{1}+\widehat{\mathbf{R}}) \\
\rightarrow & (\mathbf{1}, \mathbf{1})_{I}+(\mathbf{q}+\mathbf{1}, \mathbf{1})+\left(\mathbf{S p i n}(q+1), \operatorname{Spin}\left(Q_{q}\right)\right)+(\mathbf{1}, \mathbf{1})_{I I} \\
\rightarrow & (\mathbf{1}, \mathbf{1})_{I}+(\mathbf{q}, \mathbf{1})+(\mathbf{1}, \mathbf{1})_{I I I}+\left(\operatorname{Spin}^{\prime}(q), \operatorname{Spin}\left(Q_{q}\right)\right)+\left(\mathbf{S p i n}^{\prime \prime}(q), \operatorname{Spin}\left(Q_{q}\right)\right)+(\mathbf{1}, \mathbf{1})_{I I} .
\end{aligned}
$$

Eq. A.45 corresponds to the "branching" (recall Eq. A.36)

$$
\mathcal{Z} \equiv\left(Z, Z_{x}\right) \longrightarrow\left(Z, Z_{(\mathbf{1}, \mathbf{1})_{I I}}, Z_{(\mathbf{1}, \mathbf{1})_{I I I}}, Z_{(\mathbf{q}, \mathbf{1})}, Z_{\left(\operatorname{Spin}^{\prime}(q), \mathbf{S p i n}\left(Q_{q}\right)\right)}, Z_{\left(\mathbf{S p i n}^{\prime \prime}(q), \mathbf{S p i n}\left(Q_{q}\right)\right)}\right) .
$$

Therefore, with respect to $S O(q) \times \mathcal{A}_{q}$, besides $Z$, one obtains:

- two singlets (note that $(\mathbf{1}, \mathbf{1})_{I I}$ is a singlet of $S O(q, 1) \times \mathcal{A}_{q}$, as well);

- one vector $(\mathbf{q}, \mathbf{1})$;

- two (double-)spinors $\left(\operatorname{Spin}^{\prime}(q), \operatorname{Spin}\left(Q_{q}\right)\right)$ and $\left(\operatorname{Spin}^{\prime \prime}(q), \operatorname{Spin}\left(Q_{q}\right)\right)$.

The position which solves (with maximal - compact - symmetry $S O(q) \times \mathcal{A}_{q}$ ) the "small" lightlike $G_{5}$-invariant defining constraints 4.50) in "dressed" charges (and in a way consistent with an orbit representative having $Z=0$ ) reads as follows:

$$
\left\{\begin{array}{l}
Z \equiv Z_{(\mathbf{1}, \mathbf{1})_{I}}=0 ; \\
Z_{(\mathbf{q}, \mathbf{1})}=0 ; \\
q_{\left(\mathbf{S p i n}^{\prime}(q), \operatorname{Spin}\left(Q_{q}\right)\right)}=0 ; \\
q_{\left(\mathbf{S p i n}^{\prime \prime}(q), \operatorname{Spin}\left(Q_{q}\right)\right)}=0,
\end{array}\right.
$$

with the two singlets $Z_{(\mathbf{1}, \mathbf{1})_{I I}}$ and $Z_{(\mathbf{1}, \mathbf{1})_{I I I}}$ constrained by

$$
T_{(\mathbf{1}, \mathbf{1})_{I I}(\mathbf{1}, \mathbf{1})_{I I}(\mathbf{1}, \mathbf{1})_{I I}} Z_{(\mathbf{1}, \mathbf{1})_{I I}}^{2}+3 T_{(\mathbf{1}, \mathbf{1})_{I I}(\mathbf{1}, \mathbf{1})_{I I I}(\mathbf{1}, \mathbf{1})_{I I I}} Z_{(\mathbf{1}, \mathbf{1})_{I I I}}^{2}=0 .
$$

Besides $S O(q+1) \times \mathcal{A}_{q}$, the only other group which is maximally (and symmetrically) embedded into $\widetilde{H}_{5}$ and which has $S O(q) \times \mathcal{A}_{q}$ as $(m) c s$, is $S O(q, 1) \times \mathcal{A}_{q}$. Therefore, $S O(q, 1) \times \mathcal{A}_{q}$ is also the maximal semi-simple symmetry of $\mathcal{O}_{\text {lightlike,BPS }}$, which is thus given by Eq. (4.24).

The explicit form of Eqs. A.43-A.44 and A.45 for all $q=8,4,2,1$ classifying symmetric "magic" RSG is given below.

- $q=8\left(J_{3}^{\mathbb{D}}\right)$

$$
\begin{gathered}
E_{6(-26)}\left(\supsetneq \max F_{4(-20)}\right) \supsetneq \max S O(8,1) \stackrel{m c s}{\supsetneq} S O(8) ; \\
\mathbf{2 7}(\rightarrow \mathbf{1}+\mathbf{2 6}) \rightarrow \mathbf{1}_{I}+\mathbf{9}+\mathbf{1 6}+\mathbf{1}_{I I} \rightarrow \mathbf{1}_{I}+\mathbf{8}_{v}+\mathbf{1}_{I I I}+\mathbf{1}_{I I}+\mathbf{8}_{s}+\mathbf{8}_{c} . \\
\mathbf{-}=4\left(J_{3}^{\mathbb{H}}\right)(U S p(2,2) \sim S O(5,1), U S p(2) \sim S U(2)) \\
S U^{*}(6)(\supsetneq \max U S p(4,2)) \supsetneq \max U S p(2,2) \times U S p(2) \stackrel{m c s}{\supsetneq} U S p(2) \times U S p(2) \times U S p(2) ; \\
\mathbf{1 5}(\rightarrow \mathbf{1}+\mathbf{1 4}) \rightarrow(\mathbf{1}, \mathbf{1})_{I}+(\mathbf{5}, \mathbf{1})+(\mathbf{4}, \mathbf{2})+(\mathbf{1}, \mathbf{1})_{I I} \rightarrow \\
\left.\rightarrow(\mathbf{1}, \mathbf{1}, \mathbf{1})_{I}+(\mathbf{1}, \mathbf{1}, \mathbf{1})_{I I I}+(\mathbf{2}, \mathbf{2}, \mathbf{1})+(\mathbf{2}, \mathbf{1}, \mathbf{2})+(\mathbf{1}, \mathbf{2}, \mathbf{2})+(\mathbf{1}, \mathbf{1}, \mathbf{1}) A_{I} 51\right)
\end{gathered}
$$


- $q=2\left(J_{3}^{\mathbb{C}}\right)$

$$
\begin{aligned}
& S L(3, \mathbb{C})(\supsetneq \max S U(2,1)) \supsetneq \max S U(1,1) \times U(1) \stackrel{m c s}{\supsetneq} U(1) \times U(1) ; \\
& \mathbf{9}(\rightarrow \mathbf{1}+\mathbf{8}) \rightarrow\left(\mathbf{1}_{0}\right)_{I}+\mathbf{2}_{3}+\mathbf{2}_{-3}+\mathbf{3}_{0}+\left(\mathbf{1}_{0}\right)_{I I} \rightarrow\left(\mathbf{1}_{0}\right)_{I}+\mathbf{2}_{0}+\mathbf{2}_{3}+\mathbf{2}_{-3}+\left(\mathbf{1}_{0}\right)_{I I I}+\left(\mathbf{1}_{0}\right)_{I I} .
\end{aligned}
$$

- $q=1\left(J_{3}^{\mathbb{R}}\right)$

$$
\begin{aligned}
& S L(3, \mathbb{R})(\supsetneq \max S O(2,1)) \supsetneq \max S O(1,1) \stackrel{m c s}{\supsetneq} 1 ; \\
& \mathbf{6}^{\prime}(\rightarrow \mathbf{1}+\mathbf{5}) \rightarrow \mathbf{1}_{I}+\mathbf{2}+\mathbf{1}_{I I}+\mathbf{2} \rightarrow \mathbf{1}_{I}+\mathbf{1}_{I I}+\mathbf{1}_{I I I}+\mathbf{1}_{I V}+\mathbf{1}_{V}+\mathbf{1}_{V I},
\end{aligned}
$$

where 1 denotes the identity element.

The origin of $\mathbb{R}^{\left(\operatorname{spin}(q+1), \operatorname{spin}\left(Q_{q}\right)\right)}$ in the stabilizer of $\mathcal{O}_{\text {lightlike, } B P S}$ will be explained through the procedure of suitable İnönü-Wigner contraction performed in Sub-App. B.1

\section{B Equivalent Derivations}

In this Appendix, we determine the general form of "small" charge orbits of symmetric "magic" RSG (see Eqs. 4.14, 4.24) and 4.29) through suitable group theoretical procedures, namely:

- İnönü-Wigner contractions, for "small" lightlike orbits, Sub-App. B.1.

- $S O(1,1)$-three-grading, for "small" critical orbit, Sub-App. B.2.

Such procedures will clarify the origin of the non-semi-simple Abelian (namely, translational) factor (recall Eq. (4.1), definitions (4.17)-(4.18), and see Eq. (B.41) below)

$$
\mathcal{T}=\mathbb{R}^{\left(\operatorname{spin}(q+1), \operatorname{spin}\left(Q_{q}\right)\right)}
$$

in all three classes (lightlike BPS, lightlike non-BPS, and critical BPS) of "small" orbits (for each relevant $q=8,4,2,1)$.

\section{B.1 İnönü-Wigner Contractions}

\section{B.1.1 $\mathcal{O}_{\text {lightlike }, B P S}$}

In order to deal with $\mathcal{O}_{\text {lightlike,BPS }}$, we start from the group embedding A.33. This determines the following decompositions of irreprs. (Adj and Fund respectively denoting the adjoint and fundamental irrepr.):

$$
\operatorname{Adj}\left(G_{5}\right) \rightarrow \operatorname{Adj}\left(\widetilde{H}_{5}\right)+\operatorname{Fund}\left(\widetilde{H}_{5}\right)
$$

and further

$$
\begin{aligned}
\operatorname{Adj}\left(\widetilde{H}_{5}\right) & \rightarrow(\operatorname{Adj}(S O(q+1)), \mathbf{1})+\left(\mathbf{1}, \operatorname{Adj}\left(\mathcal{A}_{q}\right)\right)+\left(\operatorname{Spin}(q+1), \operatorname{Spin}\left(Q_{q}\right)\right)_{I} \\
\text { Fund }\left(\widetilde{H}_{5}\right) & \rightarrow(\mathbf{1}, \mathbf{1})+(\mathbf{q}+\mathbf{1}, \mathbf{1})+\left(\operatorname{Spin}(q+1), \operatorname{Spin}\left(Q_{q}\right)\right)_{I I}
\end{aligned}
$$


where trivially $\operatorname{Adj}(S O(q+1))=\frac{\mathbf{q}(\mathbf{q}+\mathbf{1})}{\mathbf{2}}$. Eqs. B.2)-B.4 thus imply

$$
\begin{aligned}
\operatorname{Adj}\left(G_{5}\right) \rightarrow & \left(\operatorname{Spin}(q+1), \operatorname{Spin}\left(Q_{q}\right)\right)_{I}+ \\
& +(\operatorname{Adj}(S O(q+1)), \mathbf{1})+\left(1, \operatorname{Adj}\left(\mathcal{A}_{q}\right)\right)+ \\
& +(\mathbf{1}, \mathbf{1})+(\mathbf{q}+\mathbf{1}, \mathbf{1})+\left(\operatorname{Spin}(q+1), \operatorname{Spin}\left(Q_{q}\right)\right)_{I I} .
\end{aligned}
$$

The decomposition of the branching (B.3) yields to

$$
\underbrace{\operatorname{Adj}\left(\widetilde{H}_{5}\right)}_{\mathfrak{g}_{\tilde{H}_{5}}} \rightarrow \underbrace{(\operatorname{Adj}(S O(q+1)), \mathbf{1})+\left(1, \operatorname{Adj}\left(\mathcal{A}_{q}\right)\right)}_{\mathfrak{h}_{\tilde{H}_{5}}}{\underset{\oplus}{\oplus_{s}}}_{\underbrace{\left(\operatorname{Spin}(q+1), \operatorname{Spin}\left(Q_{q}\right)\right)_{I}}_{\mathfrak{k}_{\tilde{H}_{5}}}} .
$$

The coset (recall Eq. 3.62)

$$
\frac{\widetilde{H}_{5}}{m c s\left(\widetilde{H}_{5}\right)}=\frac{\widetilde{H}_{5}}{S O(q+1) \times \mathcal{A}_{q}}=\mathcal{M}_{n B P S, \text { large }}
$$

is symmetric, with real dimension, Euclidean signature and character respectively (see e.g. [54, 56]; here "c" and " $n c$ " respectively stand for "compact" and "non-compact"):

$$
\begin{aligned}
& \operatorname{dim}_{\mathbb{R}}=2 q ; \\
& (c, n c)=(0,2 q) ; \\
& \chi \equiv c-n c=-2 q .
\end{aligned}
$$

By definition, the symmetricity of $\mathcal{M}_{n B P S \text {,large }}$ implies that

$$
\begin{aligned}
{\left[\mathfrak{h}_{\widetilde{H}_{5}}, \mathfrak{h}_{\widetilde{H}_{5}}\right.} & =\mathfrak{h}_{\widetilde{H}_{5}} ; \\
\mathfrak{h}_{\widetilde{H}_{5}}, \mathfrak{k}_{\widetilde{H}_{5}} & =\mathfrak{k}_{\widetilde{H}_{5}} ; \\
\left.\mathfrak{k}_{\widetilde{H}_{5}}, \mathfrak{k}_{\widetilde{H}_{5}}\right] & =\mathfrak{h}_{\widetilde{H}_{5}} .
\end{aligned}
$$

The "decoupling" of $\mathfrak{h}_{\widetilde{H}_{5}}$, with subsequent transformation of the irrepr. $\left(\mathbf{S p i n}(q+1), \mathbf{S p i n}\left(Q_{q}\right)\right)_{I}$ of $S O(q+1) \times \mathcal{A}_{q}$ into the non-semi-simple, Abelian (namely, translational) part of the stabilizer of $\mathcal{O}_{\text {lightlike,BPS }}$ is achieved by performing a uniform rescaling of the generators of $\mathfrak{k}_{\widetilde{H}_{5}}$ :

$$
\mathfrak{k}_{\widetilde{H}_{5}} \longrightarrow \lambda \mathfrak{k}_{\widetilde{H}_{5}}, \lambda \in \mathbb{R}_{0}^{+},
$$

and then by letting $\lambda \rightarrow \infty$. This amounts to performing an Inönü-Wigner (IW) contraction [57, 58, on $\mathfrak{k}_{\widetilde{H}_{5}}$. Thus (recall Eqs. 4.14) and (4.16)):

$$
\begin{aligned}
& I W\left(\mathcal{O}_{n B P S, \text { large }}=\frac{G_{5}}{\widetilde{H}_{5}}\right) \stackrel{\text { A.33) }}{\longrightarrow} \mathcal{O}_{\text {lightlike }, B P S}=\frac{G_{5}}{\left(S O(q+1) \times \mathcal{A}_{q}\right) \rtimes \mathbb{R}^{\left(\operatorname{spin}(q+1), \operatorname{spin}\left(Q_{q}\right)\right)}} ; \\
& \mathcal{T}_{\text {lightlike,BPS }} \equiv \mathbb{R}^{\left(\operatorname{spin}(q+1), \operatorname{spin}\left(Q_{q}\right)\right)} .
\end{aligned}
$$

Thus, $\mathcal{T}_{\text {lightlike,BPS }}$ given by $(\bar{B} .12)$ is the $\mathfrak{k}_{\widetilde{H}_{5}}$-part of the decomposition $(\mathrm{B} .6)$ of the Lie algebra $\mathfrak{g}_{\widetilde{H}_{5}}$ of $\widetilde{H}_{5}$ with respect to mcs $\left(\widetilde{H}_{5}\right)=S O(q+1) \times \mathcal{A}_{q}$, which then gets "decoupled" from $\mathfrak{g}_{\widetilde{H}_{5}}$ and Abelianized through the IW contraction procedure (B.10)-(B.11). 


\section{B.1.2 $\mathcal{O}_{\text {lightlike,nBPS }}$}

On the other hand, the treatment of $\mathcal{O}_{\text {lightlike,nBPS }}$ requires to start from the embedding A.43) (actually, without the last step involving $S O(q) \times \mathcal{A}_{q}=\operatorname{mcs}\left(\widehat{h}_{5}\right)$; recall Eq. A.44)):

$$
G_{5} \supsetneq \max \widetilde{H}_{5} \supsetneq \max \widehat{h}_{5}=S O(q, 1) \times \mathcal{A}_{q} .
$$

The subsequent decompositions of $\operatorname{Adj}\left(G_{5}\right), \operatorname{Adj}\left(\widetilde{H}_{5}\right)$ and Fund $\left(\widetilde{H}_{5}\right)$ are given by Eqs. B.2, (B.3) and (B.4), respectively, thus yielding the same decomposition as in (B.5). Consequently, the decomposition of the branching (B.3) yields the same result as in B.6.

The coset (recall Eq. (3.62)

$$
\frac{\widetilde{H}_{5}}{\widehat{h}_{5}}=\frac{\widetilde{H}_{5}}{S O(q, 1) \times \mathcal{A}_{q}}
$$

is symmetric, with real dimension, Euclidean signature and character respectively:

$$
\begin{aligned}
& \operatorname{dim}_{\mathbb{R}}=2 q ; \\
& (c, n c)=(q, q) ; \\
& \chi \equiv c-n c=0 .
\end{aligned}
$$

By definition, the symmetricity of $\frac{\widetilde{H}_{5}}{\widehat{h}_{5}}$ implies the same relations as in B.9.

Thus, the "decoupling" of $\mathfrak{h}_{\widetilde{H}_{5}}$, with subsequent transformation of the irrepr. $\left(\operatorname{Spin}(q+1), \operatorname{Spin}\left(Q_{q}\right)\right)_{I}$ of $S O(q, 1) \times \mathcal{A}_{q}$ into the non-semi-simple, Abelian (namely, translational) part of the stabilizer of $\mathcal{O}_{\text {lightlike, } n B P S}$ is achieved by performing a uniform rescaling of the generators of $\mathfrak{k}_{\widetilde{H}_{5}}$ as given by Eq. (B.10), and then by letting $\lambda \rightarrow \infty$. This amounts to performing an IW contraction [57, 58] on $\mathfrak{k}_{\widetilde{H}_{5}}$. Therefore, one obtains (recall Eqs. 4.24) and 4.26)):

$$
\begin{aligned}
I W\left(\mathcal{O}_{n B P S, \text { large }}\right) \stackrel{(\underline{A .43} \longrightarrow}{\longrightarrow} \mathcal{O}_{\text {lightlike }, n B P S} & =\frac{G_{5}}{\left(S O(q, 1) \times \mathcal{A}_{q}\right) \rtimes \mathbb{R}^{\left(\operatorname{spin}(q+1), \operatorname{spin}\left(Q_{q}\right)\right)}} ; \\
\mathcal{T}_{\text {lightlike }, n B P S}= & \mathcal{T}_{\text {lightlike }, B P S}=\mathbb{R}^{\left(\operatorname{spin}(q+1), \operatorname{spin}\left(Q_{q}\right)\right)} .
\end{aligned}
$$

Thus, $\mathcal{T}_{\text {lightlike,nBPS }}$ given by (B.17) is the $\mathfrak{k}_{\widetilde{H}_{5}}$-part of the decomposition (B.6) of the Lie algebra $\mathfrak{g}_{\widetilde{H}_{5}}$ of $\widetilde{H}_{5}$ with respect to $\widehat{h}_{5}=S O(q, 1) \times \mathcal{A}_{q}$, which then gets "decoupled" from $\mathfrak{g}_{\widetilde{H}_{5}}$ and Abelianized through the IW contraction procedure (see Eqs. (B.10) and (B.16).

Note that the IW contraction does not change the dimension of the starting orbit. Indeed $\mathcal{O}_{\text {lightlike,BPS }}$, obtained through the IW contraction of $\mathcal{O}_{n B P S \text {,large }}$ along the branching A.33), has the same real dimension of $\mathcal{O}_{n B P S \text {,large }}$ itself. Analogously, also $\mathcal{O}_{\text {lightlike,nBPS }}$, obtained through

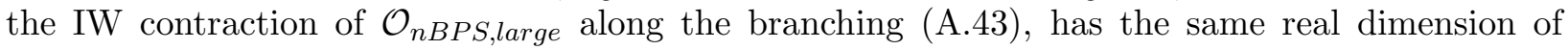
$\mathcal{O}_{n B P S, \text { large }}$ itself.

\section{B.1.3 Details}

Below, we explicit in order, besides (B.2)-(B.4), the relevant formulæ of the derivations given above, namely Eqs. (B.7), (B.8), (B.11), and (B.14), (B.15), (B.16), for all $q=8,4,2,1$ classifying symmetric "magic" RSG. 
- $q=8\left(J_{3}^{\mathbb{D}}\right)$

$$
\begin{aligned}
& 78 \rightarrow 26+52 \\
& 52 \rightarrow 36+16_{I} \\
& \mathbf{2 6} \rightarrow \mathbf{1}+\mathbf{9}+\mathbf{1 6} \mathbf{6}_{I I} \text {; } \\
& \frac{\widetilde{H}_{5}}{m c s\left(\widetilde{H}_{5}\right)}=\left.\frac{\widetilde{H}_{5}}{S O(q+1) \times \mathcal{A}_{q}}\right|_{q=8}=\mathcal{M}_{n B P S, \text { large }, J_{3}, d=5}=\frac{F_{4(-20)}}{S O(9)} ; \\
& \operatorname{dim}_{\mathbb{R}}=16 ; \quad(c, n c)=(0,16) ; \chi=-16 ; \\
& I W\left(\mathcal{O}_{n B P S, \text { large }, J_{3}^{\oplus}}=\frac{E_{6(-26)}}{F_{4(-20)}}\right) \stackrel{A \text { A.33 }}{\Longrightarrow} \mathcal{O}_{\text {lightlike }, B P S, J_{3}^{\oplus}}=\frac{E_{6(-26)}}{S O(9) \rtimes \mathbb{R}^{16}} ; \\
& \frac{\widetilde{H}_{5}}{\widehat{h}_{5}}=\left.\frac{\widetilde{H}_{5}}{S O(q, 1) \times \mathcal{A}_{q}}\right|_{q=8}=\frac{F_{4(-20)}}{S O(8,1)} \\
& \operatorname{dim}_{\mathbb{R}}=16 ; \quad(c, n c)=(8,8) ; \chi=0 ; \\
& I W\left(\mathcal{O}_{n B P S, \text { large }, J_{3}^{\oplus}}\right) \stackrel{\stackrel{A .43}{\longrightarrow}}{\longrightarrow} \mathcal{O}_{\text {lightlike, } n B P S, J_{3}^{\oplus}}=\frac{E_{6(-26)}}{S O(8,1) \times \mathbb{R}^{16}} .
\end{aligned}
$$

- $q=4\left(J_{3}^{\mathbb{H}}\right)$

$$
\begin{aligned}
& 35 \rightarrow 14+21 \text {; } \\
& 21 \rightarrow(4,2)_{I}+(10,1)+(1,3) ; \\
& 14 \rightarrow(1,1)+(5,1)+(4,2)_{I I} \text {; } \\
& \frac{\widetilde{H}_{5}}{m c s\left(\widetilde{H}_{5}\right)}=\left.\frac{\widetilde{H}_{5}}{S O(q+1) \times \mathcal{A}_{q}}\right|_{q=4}=\mathcal{M}_{n B P S, \text { large }, J_{3}^{\mathbb{H}}, d=5}=\frac{U S p(4,2)}{U S p(4) \times U S p(2)} ; \\
& \operatorname{dim}_{\mathbb{R}}=8 ; \quad(c, n c)=(0,8) ; \chi=-8 ; \\
& I W\left(\mathcal{O}_{n B P S, \text { large }, J_{3}^{\mathbb{H}}}=\frac{S U^{*}(6)}{U S p(4,2)}\right) \stackrel{\overrightarrow{A .33}}{\longrightarrow} \mathcal{O}_{\text {lightlike }, B P S, J_{3}^{\mathbb{H}}}=\frac{S U^{*}(6)}{(S O(5) \times S O(3)) \rtimes \mathbb{R}^{(4,2)}} \\
& \frac{\widetilde{H}_{5}}{\widehat{h}_{5}}=\left.\frac{\widetilde{H}_{5}}{S O(q, 1) \times \mathcal{A}_{q}}\right|_{q=4}=\frac{U S p(4,2)}{U S p(2,2) \times U S p(2)} \\
& \operatorname{dim}_{\mathbb{R}}=8 ; \quad(c, n c)=(4,4) ; \chi=0 ; \\
& I W\left(\mathcal{O}_{n B P S, \text { large }, J_{3}^{\mathbb{H}}} \stackrel{\stackrel{A .43}{\longrightarrow}}{\longrightarrow} \mathcal{O}_{\text {lightlike }, n B P S, J_{3}^{\mathbb{H}}}=\frac{S U^{*}(6)}{(S O(4,1) \times S O(3)) \rtimes \mathbb{R}^{(4,2)}} .\right.
\end{aligned}
$$

- $q=2\left(J_{3}^{\mathbb{C}}\right)$. Notice that in this case Eq. B.2 gets modified into

$$
\begin{aligned}
& \operatorname{Adj}\left(G_{5}\right) \rightarrow \operatorname{Adj}\left(\widetilde{H}_{5}\right)+\operatorname{Adj}\left(\widetilde{H}_{5}\right) ; \\
& \mathbf{1 6} \rightarrow \mathbf{8}+\mathbf{8} ; \\
& \mathbf{8} \rightarrow \mathbf{3}_{0}+\mathbf{1}_{0}+\mathbf{2}_{3}+\mathbf{2}_{-3} .
\end{aligned}
$$


Everything fits also because for $q=2$ it holds that

$$
\begin{aligned}
& (\mathbf{q}+\mathbf{1}, \mathbf{1})=(\mathbf{A d j}(S O(q+1)), \mathbf{1})=\mathbf{3}_{0} ; \\
& \left(\mathbf{1}, \operatorname{Adj}\left(\mathcal{A}_{q}\right)\right)=(\mathbf{1}, \mathbf{1})=\mathbf{1}_{0} . \\
& \frac{\widetilde{H}_{5}}{m c s\left(\widetilde{H}_{5}\right)}=\left.\frac{\widetilde{H}_{5}}{S O(q+1) \times \mathcal{A}_{q}}\right|_{q=2}=\mathcal{M}_{n B P S, \text { large }, J_{3}^{\mathbb{C}}, d=5}=\frac{S U(2,1)}{S U(2) \times U(1)} ; \\
& \operatorname{dim}_{\mathbb{R}}=4 ; \quad(c, n c)=(0,4) ; \chi=-4 ; \\
& I W\left(\mathcal{O}_{n B P S, \text { large }, J_{3}^{\mathbb{C}}}=\frac{S L(3, \mathbb{C})}{S U(2,1)}\right) \stackrel{A .33}{\Longrightarrow} \mathcal{O}_{\text {lightlike }, B P S, J_{3}^{\mathbb{C}}}=\frac{S L(3, \mathbb{C})}{(S O(3) \times S O(2)) \rtimes \mathbb{R}^{(2,2)}} . \\
& \frac{\widetilde{H}_{5}}{\widehat{h}_{5}}=\left.\frac{\widetilde{H}_{5}}{S O(q, 1) \times \mathcal{A}_{q}}\right|_{q=2}=\frac{S U(2,1)}{S U(1,1) \times U(1)} \\
& \operatorname{dim}_{\mathbb{R}}=4 ; \quad(c, n c)=(2,2) ; \chi=0 ; \\
& I W\left(\mathcal{O}_{n B P S, \text { large }, J_{3}^{\mathbb{C}}}\right) \stackrel{\overrightarrow{A .43}}{\underline{\longrightarrow}} \mathcal{O}_{\text {lightlike }, n B P S, J_{3}^{\mathbb{C}}}=\frac{S L(3, \mathbb{C})}{(S O(2,1) \times S O(2)) \times \mathbb{R}^{(2,2)}} .
\end{aligned}
$$

- $q=1\left(J_{3}^{\mathbb{R}}\right)$. Notice that in this case Eq. B.2 gets modified into

$$
\begin{aligned}
& \operatorname{Adj}\left(G_{5}\right) \rightarrow \operatorname{Adj}\left(\widetilde{H}_{5}\right)+\operatorname{Spin}_{s=2}\left(\widetilde{H}_{5}\right) \\
& \mathbf{8} \rightarrow \mathbf{3}+\mathbf{5} \\
& \mathbf{3} \rightarrow \mathbf{1}_{I I}+\mathbf{2}_{I} \\
& \mathbf{5} \rightarrow \mathbf{1}_{I}+\mathbf{2}_{I I I}+\mathbf{2}_{I I} ;
\end{aligned}
$$

Everything fits also because for $q=1$ it holds that

$$
\begin{aligned}
& (\mathbf{q}+\mathbf{1}, \mathbf{1})=(\mathbf{A d j}(S O(q+1)), \mathbf{1})=\mathbf{2} ; \\
& \left(\mathbf{1}, \operatorname{Adj}\left(\mathcal{A}_{q}\right)\right)=(\mathbf{1}, \mathbf{1})=\mathbf{1} . \\
& \frac{\widetilde{H}_{5}}{m c s\left(\widetilde{H}_{5}\right)}=\left.\frac{\widetilde{H}_{5}}{S O(q+1) \times \mathcal{A}_{q}}\right|_{q=1}=\mathcal{M}_{n B P S, \text { large }, J_{3}^{\mathbb{R}}, d=5}=\frac{S O(2,1)}{S O(2)} \sim \frac{S U(1,1)}{U(1)} ; \\
& \operatorname{dim}_{\mathbb{R}}=2 ; \quad(c, n c)=(0,2) ; \chi=-2 ; \\
& I W\left(\mathcal{O}_{n B P S, \text { large }, J_{3}^{\mathbb{R}}}=\frac{S L(3, \mathbb{R})}{S O(2,1)}\right) \stackrel{\overrightarrow{A .33}}{\longrightarrow} \mathcal{O}_{\text {lightlike }, B P S, J_{3}^{\mathbb{R}}}=\frac{S L(3, \mathbb{R})}{S O(2) \rtimes \mathbb{R}^{2}} . \\
& \frac{\widetilde{H}_{5}}{\widehat{h}_{5}}=\left.\frac{\widetilde{H}_{5}}{S O(q, 1) \times \mathcal{A}_{q}}\right|_{q=1}=\frac{S O(2,1)}{S O(1,1)} \\
& \operatorname{dim}_{\mathbb{R}}=2 ; \quad(c, n c)=(1,1) ; \chi=0 ; \\
& I W\left(\mathcal{O}_{n B P S, \text { large }, J_{3}^{\mathbb{R}}}\right) \stackrel{\text { A.43 }}{\longrightarrow} \mathcal{O}_{\text {lightlike }, n B P S, J_{3}^{\mathbb{R}}}=\frac{S L(3, \mathbb{R})}{(S O(1,1)) \rtimes \mathbb{R}^{2}} .
\end{aligned}
$$




\section{B.2 $S O(1,1)$-Three Grading and $\mathcal{O}_{\text {critical }, B P S}$}

In order to deal with $\mathcal{O}_{\text {critical,BPS }}$, we start from the group embedding (A.1). As pointed out above, this is the unique maximal embedding (at least among the symmetric ones; see e.g. [54]) into $G_{5}$ to exhibit a commuting factor $S O(1,1)$.

Therefore, the Lie algebra $\mathfrak{g}_{G_{5}}$ of $G_{5}$ admits a three-grading with respect to the Lie algebra $\mathbb{R}$ of $S O(1,1)$ as follows:

$$
\mathfrak{g}_{G_{5}}=\mathcal{W}^{+3} \oplus_{s} \mathcal{W}^{0} \oplus_{s} \mathcal{W}^{-3}
$$

where as above the subscripts denote the weights with respect to $S O(1,1)$ itself. At the level of "branching" of $\mathbf{A d j}\left(G_{5}\right)$, the $S O(1,1)$-three-grading reads as follows:

$$
\begin{aligned}
\operatorname{Adj}\left(G_{5}\right) \rightarrow & (\mathbf{1}, \mathbf{1})_{0}+\left(\operatorname{Adj}\left(G_{6}\right), \mathbf{1}\right)_{0}+\left(\mathbf{1}, \mathbf{A d j}\left(\mathcal{A}_{q}\right)\right)_{0}+ \\
& +\left(\operatorname{Spin}(q+2), \operatorname{Spin}\left(Q_{q}\right)\right)_{-3}+ \\
& +\left(\operatorname{Spin}^{\prime}(q+2), \operatorname{Spin}\left(Q_{q}\right)\right)_{+3} .
\end{aligned}
$$

Thus, the decomposition $\mathrm{B} .33$ yields the following identification of the graded terms in $(\mathrm{B} .32)$ :

$$
\begin{aligned}
& \mathcal{W}^{0} \equiv(\mathbf{1}, \mathbf{1})_{0} \quad+\left(\mathbf{A d j}\left(G_{6}\right), \mathbf{1}\right)_{0}+\left(\mathbf{1}, \mathbf{A d j}\left(\mathcal{A}_{q}\right)\right)_{0} \\
& \downarrow_{\exp } \quad \downarrow_{\exp } \quad \downarrow \text { exp } \\
& S O(1,1) \quad G_{6} \quad \mathcal{A}_{q} \\
& \mathcal{W}^{+3} \equiv\left(\operatorname{Spin}^{\prime}(q+2), \operatorname{Spin}\left(Q_{q}\right)\right)_{+3} ; \\
& \mathcal{W}^{-3} \equiv\left(\operatorname{Spin}(q+2), \operatorname{Spin}\left(Q_{q}\right)\right)_{-3} \text {, }
\end{aligned}
$$

with "exp" denoting the exponential mapping.

Thus, $\mathcal{O}_{\text {critical }, B P S}$ is obtained by cosetting $G_{5}$ with the +3 (or equivalently -3 )-graded extension of $\mathcal{W}^{0}-(\mathbf{1}, \mathbf{1})_{0}$, namely:

$$
\mathcal{O}_{\text {critical }, B P S}=\frac{G_{5}}{\mathcal{N}_{+3(-3)}}
$$

where

$$
\begin{aligned}
\mathcal{N}_{+3} & \equiv \exp \left[\left(\mathcal{W}^{0}-(\mathbf{1}, \mathbf{1})_{0}\right) \oplus_{s} \mathcal{W}^{+3}\right] \\
& =\exp \left[\left(\left(\mathbf{A d j}\left(G_{6}\right), \mathbf{1}\right)_{0}+\left(\mathbf{1}, \mathbf{A d j}\left(\mathcal{A}_{q}\right)\right)_{0}\right) \oplus_{s}\left(\mathbf{S p i n}^{\prime}(q+2), \mathbf{S p i n}\left(Q_{q}\right)\right)_{+3}\right] \\
& =\left(G_{6} \times \mathcal{A}_{q}\right) \rtimes \mathbb{R}^{\left(s p i n(q+2), \operatorname{spin}\left(Q_{q}\right)\right)} ; \\
\mathcal{N}_{-3} & \equiv \exp \left[\left(\mathcal{W}^{0}-(\mathbf{1}, \mathbf{1})_{0}\right) \oplus_{s} \mathcal{W}^{-3}\right] \\
& =\exp \left[\left(\left(\mathbf{A d j}\left(G_{6}\right), \mathbf{1}\right)_{0}+\left(\mathbf{1}, \mathbf{A d j}\left(\mathcal{A}_{q}\right)\right)_{0}\right) \oplus_{s}\left(\mathbf{S p i n}(q+2), \operatorname{Spin}\left(Q_{q}\right)\right)_{-3}\right] \\
& =\left(G_{6} \times \mathcal{A}_{q}\right) \rtimes \mathbb{R}^{\left(\operatorname{spin}(q+2), \operatorname{spin}\left(Q_{q}\right)\right)} .
\end{aligned}
$$

Thus, it holds that Eqs. (B.37) and (B.38) (or equivalently Eqs. (B.37) and (B.39) ) are consistent with the general form of $\mathcal{O}_{\text {critical,BPS }}$ given by Eq. (4.29).

Therefore, in the stabilizer of $\mathcal{O}_{\text {critical }, B P S}$, the factor

$$
\mathcal{T}_{\text {critical }, B P S}=\mathbb{R}^{\left(\operatorname{spin}(q+2), \operatorname{spin}\left(Q_{q}\right)\right)}=\mathbb{R}^{\left(\operatorname{spin}(q+1), \operatorname{spin}\left(Q_{q}\right)\right)}
$$


is given by the exponential mapping of the Abelian subalgebra of $\mathfrak{g}_{G_{5}}$ contained into the +3 (or equivalently -3)-graded extension of $\mathcal{W}^{0}-(\mathbf{1}, \mathbf{1})_{0}$ through the $S O(1,1)$-three grading $(\mathrm{B} .32)$, corresponding to the irrepr. $\left(\boldsymbol{S}_{\operatorname{pin}}{ }^{\prime}(q+2), \operatorname{Spin}\left(Q_{q}\right)\right)_{+3}\left(\right.$ or equivalently $\left.\left(\operatorname{Spin}(q+2), \boldsymbol{S} \mathbf{P i n}\left(Q_{q}\right)\right)_{-3}\right)$ of $G_{6} \times \mathcal{A}_{q}(\times S O(1,1))$.

The results obtained in Sub-Apps. B.1 and B.2 (and reported in Tables 3 and 4) allows one to conclude that all "small" charge orbits of symmetric "magic" RSG (classified by $q=8,4,2,1$ ) share the same non-semi-simple, Abelian (namely, translational) part of the stabilizer. Namely, Eqs. (B.17) and (B.40) yield to:

$$
\mathcal{T}_{\text {lightlike }, B P S}=\mathcal{T}_{\text {lightlike }, n B P S}=\mathcal{T}_{\text {critical }, B P S}=\mathbb{R}^{\left(\operatorname{spin}(q+1), \operatorname{spin}\left(Q_{q}\right)\right)} .
$$

\section{B.2.1 Details}

Below, we explicit Eqs. B.33)-B.36 for all $q=8,4,2,1$ classifying symmetric "magic" RSG.

- $q=8\left(J_{3}^{\mathbb{Q}}\right)$

$$
\mathbf{7 8} \rightarrow \overbrace{\mathbf{1}_{0}+\mathbf{4 5}_{0}}^{\mathcal{W}^{0}}+\overbrace{\mathbf{1 6} \mathbf{W}_{-3}}^{\mathcal{W}^{-3}}+\overbrace{\mathbf{1 6 _ { + 3 } ^ { \prime }}}^{\mathcal{W}_{+3}^{+3}} .
$$

- $q=4\left(J_{3}^{\mathbb{H}}\right)$

$$
\mathbf{3 5} \rightarrow \overbrace{(\mathbf{1 , 1})_{0}+(\mathbf{1 5}, \mathbf{1})_{0}+(\mathbf{1}, \mathbf{3})_{0}}^{\mathcal{W}^{0}}+\overbrace{(\mathbf{4 , 2})_{-3}}^{\mathcal{W}^{-3}}+\overbrace{(\mathbf{4 , 2})_{+3}}^{\mathcal{W}^{+3}} .
$$

- $q=2\left(J_{3}^{\mathbb{C}}\right)$. In this case it should be recalled that

$$
\operatorname{Adj}(S L(3, \mathbb{C}))=\mathbf{1 6} \equiv \mathbf{3} \times \mathbf{3}^{\prime}+\overline{\mathbf{3}} \times \overline{\mathbf{3}}^{\prime}-2 \text { singlets } .
$$

Thus, by recalling Eqs. A.22 - A.22, one can compute that under $S L(3, \mathbb{C}) \supsetneq \max S L(2, \mathbb{C}) \times$ $S L(1, \mathbb{C}) \times G L(1, \mathbb{C}):$

$$
\begin{aligned}
& \mathbf{3} \times \mathbf{3}^{\prime} \rightarrow\left(\mathbf{3}_{0}\right)_{0}+\left(\mathbf{1}_{0}\right)_{0}+\left(\mathbf{2}_{3}\right)_{-3}+\left(\overline{\mathbf{2}}_{-3}\right)_{3}+\left(\mathbf{1}_{0}\right)_{0} ; \\
& \overline{\mathbf{3}} \times \overline{\mathbf{3}}^{\prime} \rightarrow\left(\mathbf{3}_{0}\right)_{0}+\left(\mathbf{1}_{0}\right)_{0}+\left(\overline{\mathbf{2}}_{-3}\right)_{-3}+\left(\mathbf{2}_{3}\right)_{3}+\left(\mathbf{1}_{0}\right)_{0} .
\end{aligned}
$$

Therefore:

$$
\operatorname{Adj}(S L(3, \mathbb{C}))=\mathbf{1 6} \rightarrow \overbrace{2\left(\mathbf{3}_{0}\right)_{0}+2\left(\mathbf{1}_{0}\right)_{0}}^{\mathcal{W}^{0}}+\overbrace{\left(\mathbf{2}_{3}\right)_{-3}+\left(\overline{\mathbf{2}}_{-3}\right)_{-3}}^{\mathcal{W}^{-3}}+\overbrace{\left(\mathbf{2}_{3}\right)_{+3}+\left(\overline{\mathbf{2}}_{-3}\right)_{+3}}^{\mathcal{W}^{+3}} .
$$

- $q=1\left(J_{3}^{\mathbb{R}}\right)$

$$
\mathbf{8} \rightarrow \overbrace{\mathbf{1}_{0}+\mathbf{3}_{0}}^{\mathcal{W}^{0}}+\overbrace{\mathbf{2}_{-3}}^{\mathcal{W}^{-3}}+\overbrace{\mathbf{2}_{+3}}^{\mathcal{W}^{+3}} .
$$




\section{References}

[1] G. W. Gibbons and P. K. Townsend, Vacuum interpolation in supergravity via super p-branes, Phys. Rev. Lett. 71, 3754 (1993), hep-th/9307049.

[2] C. Hull and P. K. Townsend, Unity of Superstring Dualities, Nucl. Phys. B438, 109 (1995), hep-th/9410167.

[3] S. Ferrara, R. Kallosh, A. Strominger, $\mathcal{N}=2$ extremal black holes, Phys. Rev. D52, 5412 (1995).

[4] A. Strominger, Macroscopic entropy of $\mathcal{N}=2$ extremal black holes, Phys. Lett. B383, 39 (1996).

[5] S. Ferrara, R. Kallosh, Supersymmetry and attractors, Phys. Rev. D54, 1514 (1996). S. Ferrara, R. Kallosh, Universality of supersymmetric attractors, Phys. Rev. D54, 1525 (1996).

[6] S. Ferrara, G. W. Gibbons and R. Kallosh, Black Holes and Critical Points in Moduli Space, Nucl. Phys. B500, 75 (1997), hep-th/9702103.

[7] F. Denef and G. W. Moore, Split States, Entropy Enigmas, Holes and Halos, arXiv:0702146 [hep-th].

[8] B. Bates and F. Denef, Exact Solutions for Supersymmetric Stationary Black Hole Composites, hep-th/0304094.

[9] A. Sen, Black Hole Entropy Function, Attractors and Precision Counting of Microstates, Gen. Rel. Grav. 40, 2249 (2008), arXiv:0708.1270 [hep-th].

[10] H. Ooguri, A. Strominger and C. Vafa, Black Hole Attractors and the Topological String, Phys. Rev. D70, 106007 (2004), hep-th/0405146.

[11] B. Pioline, Lectures on black holes, topological strings and quantum attractors, Class. Quant. Grav. 23, S981 (2006), hep-th/0607227.

[12] P. Kraus and F. Larsen, Attractors and Black Rings, Phys. Rev. D72, 024010 (2005), hep-th/0503219. A. Castro, J. L. Davis, P. Kraus and F. Larsen, 5D attractors with higher derivatives, JHEP 0704, 091 (2007), hep-th/0702072.

[13] A. Ceresole and G. Dall'Agata, Flow Equations for Non-BPS Extremal Black Holes, JHEP 0703, 110 (2007), hep-th/0702088. G. Lopes Cardoso, A. Ceresole, G. Dall'Agata, J. M. Oberreuter and J. Perz, First-order flow equations for extremal black holes in very special geometry, JHEP 0710, 063 (2007), arXiv:0706.3373 [hep-th].

[14] S. Ferrara and J. M. Maldacena, Branes, Central Charges and U-Duality Invariant BPS Conditions, Class. Quant. Grav. 15, 749 (1998), hep-th/9706097.

[15] S. Ferrara and M. Günaydin, Orbits of exceptional groups, duality and BPS states in string theory, Int. J. Mod. Phys. A13, 2075 (1998), hep-th/9708025.

[16] H. Lu, C. N. Pope and K. S. Stelle, Multiplet Structures of BPS Solitons, Class. Quant. Grav. 15, 537 (1998), hep-th/9708109. 
[17] L. Andrianopoli, R. D'Auria and S. Ferrara, Five-dimensional U duality, black hole entropy and topological invariants, Phys. Lett. B411, 39 (1997), hep-th/9705024.

[18] R. D'Auria, S. Ferrara, M. A. Lledó, On central charges and Hamiltonians for 0-brane dynamics, Phys. Rev. D60, 084007 (1999), hep-th/9903089.

[19] S. Ferrara and M. Günaydin, Orbits and Attractors for $\mathcal{N}=2$ Maxwell-Einstein Supergravity Theories in Five Dimensions, Nucl. Phys. B759, 1 (2006), hep-th/0606108.

[20] L. Andrianopoli, S. Ferrara, A. Marrani and M. Trigiante, Non-BPS Attractors in $5 d$ and $6 d$ Extended Supergravity, Nucl. Phys. B795, 428 (2008), arXiv:0709.3488.

[21] B. L. Cerchiai, S. Ferrara, A. Marrani and B. Zumino, Duality, Entropy and ADM Mass in Supergravity, Phys. Rev. D79, 125010 (2009), arXiv:0902.3973.

[22] S. Ferrara and A. Marrani, $\mathcal{N}=8$ non-BPS Attractors, Fixed Scalars and Magic Supergravities, Nucl. Phys. B788, 63 (2008), arXiv:0705.3866 [hep-th].

[23] S. Ferrara and A. Marrani, On the Moduli Space of non-BPS Attractors for $\mathcal{N}=2$ Symmetric Manifolds, Phys. Lett. B652, 111 (2007), arXiv:0706.1667.

[24] A. Ceresole, S. Ferrara, A. Gnecchi and A. Marrani, More on $\mathcal{N}=8$ Attractors, Phys. Rev. D80, 045020 (2009), arXiv:0904.4506 [hep-th].

[25] A. Ceresole, S. Ferrara and A. Gnecchi, 5d/4d U-dualities and $\mathcal{N}=8$ black holes, Phys. Rev. D80, 125033 (2009), arXiv:0908.1069.

[26] L. Borsten, D. Dahanayake, M. J. Duff, S. Ferrara, A. Marrani and W. Rubens, Observations on Integral and Continuous $U$-duality Orbits in $\mathcal{N}=8$ Supergravity, arXiv:1002.4223 [hep-th].

[27] J. D. Bekenstein, Phys. Rev. D7, 2333 (1973). S. W. Hawking, Phys. Rev. Lett. 26, 1344 (1971); in C. DeWitt, B. S. DeWitt, Black Holes (Les Houches 1972) (Gordon and Breach, New York, 1973). S. W. Hawking, Nature 248, 30 (1974). S. W. Hawking, Comm. Math. Phys. 43, 199 (1975).

[28] M. Günaydin, G. Sierra and P. K. Townsend, Exceptional Supergravity Theories and the Magic Square, Phys. Lett. B133, 72 (1983).

[29] M. Günaydin, G. Sierra and P. K. Townsend, The Geometry of $\mathcal{N}=2$ Maxwell-Einstein Supergravity and Jordan Algebras, Nucl. Phys. B242, 244 (1984).

[30] M. Günaydin, G. Sierra and P. K. Townsend, Gauging the $d=5$ Maxwell-Einstein Supergravity Theories: More on Jordan Algebras, Nucl. Phys. B253, 573 (1985).

[31] M. Günaydin, G. Sierra and P. K. Townsend, More on $d=5$ Maxwell-Einstein Supergravity : Symmetric Spaces and Kinks, Class. Quant. Grav. 3, 763 (1986).

[32] B. de Wit, F. Vanderseypen and A. Van Proeyen, Symmetry structure of special geometries, Nucl. Phys. B400, 463 (1993), hep-th/9210068.

[33] A. Ceresole and G. Dall'Agata, General matter coupled $\mathcal{N}=2, d=5$ gauged supergravity, Nucl. Phys. B585, 143 (2000), hep-th/0004111. 
[34] A. Ceresole, S. Ferrara and A. Marrani, 4d/5d Correspondence for the Black Hole Potential and its Critical Points, Class. Quant. Grav. 24, 5651 (2007), arXiv:0707.0964.

[35] B. de Wit and A. Van Proeyen, Symmetries of dual quaternionic manifolds, Phys. Lett. B252, $221(1990)$.

[36] B. de Wit and A. Van Proeyen, Special geometry, cubic polynomials and homogeneous quaternionic spaces, Commun. Math. Phys. 149, 307 (1992), hep-th/9112027.

[37] D. Dai-Wai Bao, Shiing-Shen Chern and Z. Shen, "An Introduction to Riemann-Finsler Geometry" (Springer-Verlag, Berlin-Heidelberg, 2000).

[38] B. Chow, P. Lu and L. Ni, "Hamilton's Ricci Flow" (American Mathematical Society, 2006).

[39] S. Bellucci, S. Ferrara, R. Kallosh and A. Marrani, Extremal Black Hole and Flux Vacua Attractors, Lect. Notes Phys. 755, 115 (2008), arXiv:0711.4547 [hep-th].

[40] S. Bellucci, A. Marrani and R. Roychowdhury, On Quantum Special Kähler Geometry, Int. J. Mod. Phys. A25, 1891 (2010), arXiv:0910.4249 [hep-th].

[41] B. de Wit and A. Van Proeyen, Broken sigma model isometries in very special geometry, Phys. Lett. B293, 94 (1992), hep-th/9207091.

[42] E. Cremmer and A. Van Proeyen, Classification of Kähler Manifolds in $\mathcal{N}=2$ Vector Multiplet Supergravity Couplings, Class. Quant. Grav. 2, 445 (1985).

[43] R. Kallosh, New Attractors, JHEP 0512, 022 (2005), hep-th/0510024.

[44] L. Andrianopoli, R. D'Auria, S. Ferrara and M. Trigiante, Extremal black holes in supergravity, Lect. Notes Phys. 737, 661 (2008), hep-th/0611345.

[45] R. D'Auria, S. Ferrara and M. Trigiante, Critical points of the Black-Hole potential for homogeneous special geometries, JHEP 0703, 097 (2007), hep-th/0701090.

[46] S. Ferrara and A. Marrani, Symmetric Spaces in Supergravity, in : "Symmetry in Mathematics and Physics", (D. Babbitt, V. Vijayanthi and R. Fioresi Eds.), Contemporary Mathematics 490, American Mathematical Society, Providence 2009, arXiv:0808.3567.

[47] M. Günaydin, Lectures on Spectrum Generating Symmetries and U-duality in Supergravity, Extremal Black Holes, Quantum Attractors and Harmonic Superspace, arXiv:0908.0374 [hep-th].

[48] E. Bergshoeff, W. Chemissany, A. Ploegh, M. Trigiante and T. Van Riet, Generating Geodesic Flows and Supergravity Solutions, Nucl. Phys. B812, 343 (2009), arXiv:0806. 2310.

[49] P. Jordan, J. Von Neumann and E. Wigner, On an algebraic generalization of the quantum mechanical formalism, Ann. Math. 35, 29 (1934).

[50] E. G. Gimon, F. Larsen and J. Simon, Black Holes in Supergravity: the non-BPS Branch, JHEP 0801, 040 (2008), arXiv:0710.4967 [hep-th].

[51] S. Bellucci, S. Ferrara, A. Marrani and A. Yeranyan, stu Black Holes Unveiled, Entropy 2008, Vol. 10(4), 507, arXiv:0808.3567 [hep-th]. 
[52] L. Andrianopoli, R. D'Auria, S. Ferrara and M. Trigiante, Fake Superpotential for Large and Small Extremal Black Holes, arXiv:1002.4340 [hep-th].

[53] G. Bossard, Y. Michel and B. Pioline, Extremal Black Holes, Nilpotent Orbits and the True Fake Superpotential, JHEP 1001, 038 (2010), arXiv:0908.1742 [hep-th].

[54] R. Gilmore, "Lie Groups, Lie Algebras, and Some of Their Applications" (Dover Publications, 2006).

[55] R. Slansky, Group Theory for Unified Model Building, Phys. Rept. 79, 1 (1981).

[56] S. Helgason, Differential Geometry, Lie Groups and Symmetric Spaces (Academic Press, New York, 1978).

[57] E. İnönü and E. P. Wigner, Proc. Nat. Acad. Sci. 39, 510 (1953).

[58] E. İnönü and E. P. Wigner, Proc. Nat. Acad. Sci. 40, 119 (1954).

[59] D. Roest and H. Samtleben, Twin Supergravities, Class. Quant. Grav. 26, 155001 (2009), arXiv:0904.1344 [hep-th].

[60] L. Andrianopoli, R. D'Auria and S. Ferrara, Central extension of extended supergravities in diverse dimensions, Int. J. Mod. Phys. A12, 3759 (1997), hep-th/9608015.

[61] L. Andrianopoli, R. D'Auria and S. Ferrara, U duality and central charges in various dimensions revisited, Int. J. Mod. Phys. A13, 431 (1998), hep-th/9612105. 